GEOLOGICAL SURVEY CIRCULAR 896-D

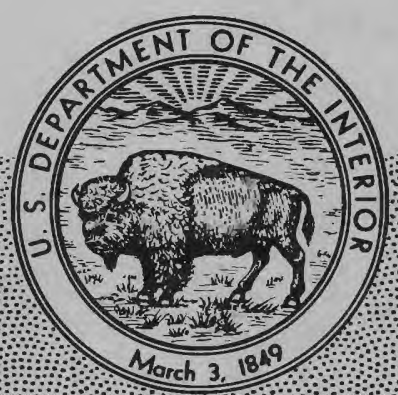

Earthquakes

in the United States

October-December 1982 


\section{Earthquakes}

\section{in the United States \\ October-December 1982}

By C. W. Stover, J. H. Minsch, F. W. Baldwin, and L. R. Brewer

GEOLOGICAL SURVEY CIRCULAR $896-D$ 
United States Department of the Interior WILLIAM P. CLARK, Secretary

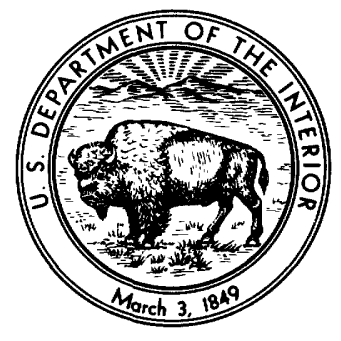

Geological Survey

Dallas L. Peck, Director 


\section{CONTENTS}

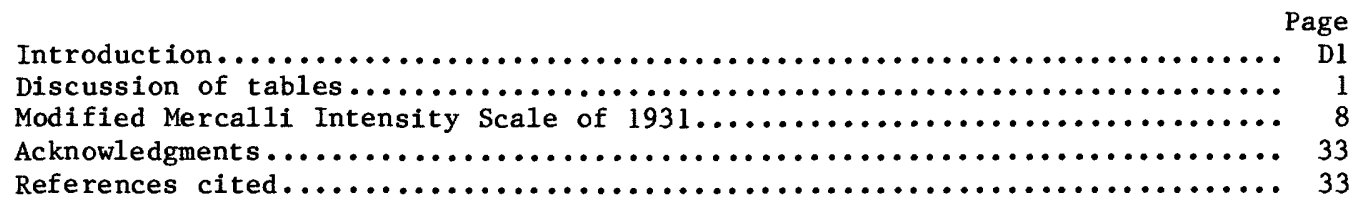

\section{ILLUSTRATIONS}

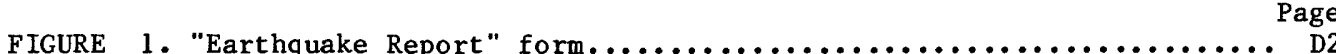

2. Map showing standard time zones of the conterminous United States. 4

3. Map showing standard time zones of Alaska and Hawaii.......... 5

4. Map of earthquake epicenters in the conterminous United States for

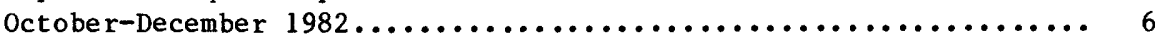

5. Map of earthquake epicenters in Alaska for October-December 1982.. 7

6. Map of earthquake epicenters in Hawaii for October-December 1982.. 8

7. Isoseismal map for the southern California earthquake of 1 October

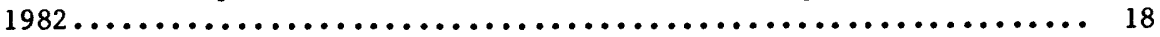

8. Isoseismal map for the central California earthquake of 25 October

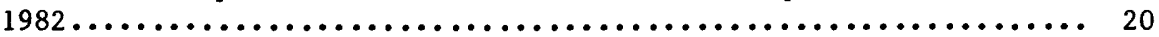

9. Isoseismal map for the northern California earthquake of 16 December 1982 .......................................... 24

10. Isoseismal map for the southeastern Idaho earthquake of 14 October

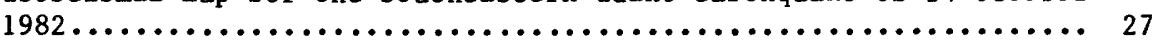

11. Isoseisma1 map for the southeastern South Dakota earthquake of 15 November $1982 . \ldots \ldots \ldots \ldots \ldots \ldots \ldots \ldots \ldots \ldots \ldots \ldots \ldots \ldots \ldots \ldots \ldots \ldots \ldots$ 


\section{TABLES}

TABLE 1. Summary of United States earthquakes for October-December 1982:

Page

Alaska...................................... D10

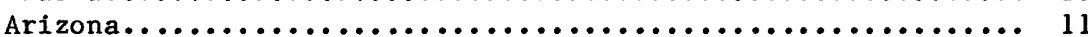

Arkansas...................................... 11

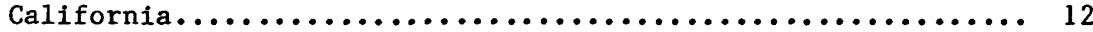

California--off the coast........................ 13

Colorado...................................... 14

Georgia...................................... 14

Hawaii ........................................ 14

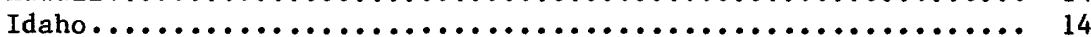

Maine......................................... 15

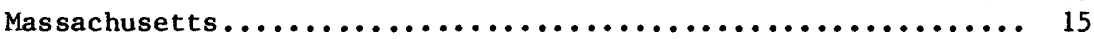

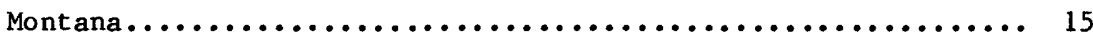

Nevada........................................ 15

New Hampshire.................................. 15

New Mexico....................................... 15

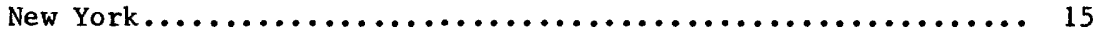

oregon......................................... 15

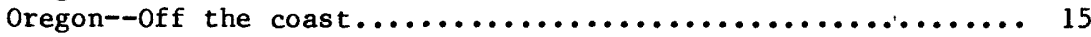

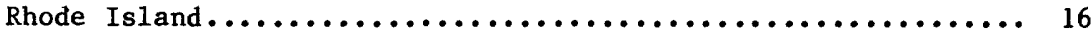

South Dakota.................................. 16

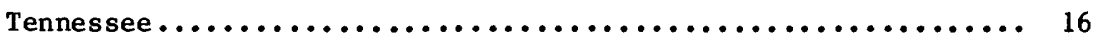

Texas ......................................... 16

Washington--Off the coast.......................... 16

Wyoming $\ldots \ldots \ldots \ldots \ldots \ldots \ldots \ldots \ldots \ldots \ldots \ldots \ldots \ldots \ldots \ldots \ldots \ldots \ldots \ldots \ldots \ldots$

Page

2. Summary of macroseismic data for United States earthquakes, October-December 1982:

Alabama ...................................... D17

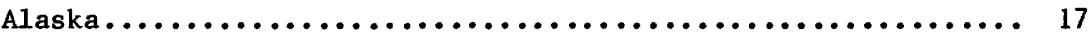

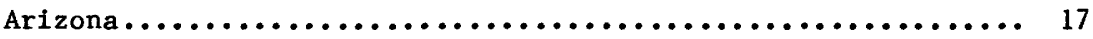

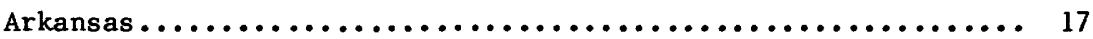

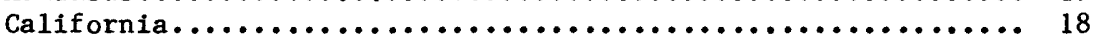

California-- off the coast......................... 25

Colorado..................................... 25

Georgia...................................... 26

Hawaii....................................... 26

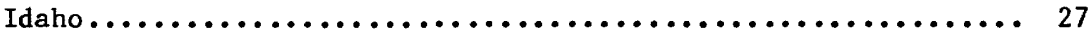

Iowa ........................................... 29

Maine......................................... 29

Mont ana........................................ 29

Nebraska.......................................... 29

Nevada .......................................... 29

New Hampshire.................................... 29

New Mexico..................................... 30

oregon $\ldots \ldots \ldots \ldots \ldots \ldots \ldots \ldots \ldots \ldots \ldots \ldots \ldots \ldots \ldots \ldots \ldots \ldots \ldots \ldots \ldots \ldots \ldots \ldots . \ldots . \ldots \ldots$

Rhode Island................................... 30

South Dakota.................................... 30

Tennessee....................................... 31

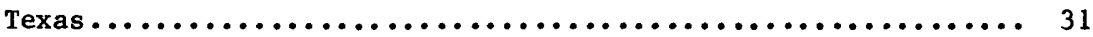

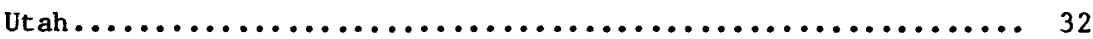

Vermont $\ldots \ldots \ldots \ldots \ldots \ldots \ldots \ldots \ldots \ldots \ldots \ldots \ldots \ldots \ldots \ldots \ldots \ldots \ldots \ldots \ldots \ldots, 32$

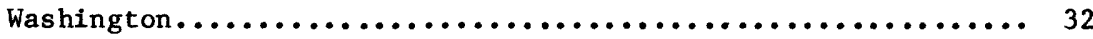

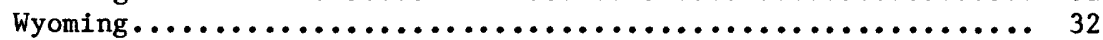




\title{
Earthquakes in the United States, October-December 1982
}

\author{
By C. W. Stover, J. H. Minch, F. W. Baldwin, and L. R. Brewer
}

\section{INTRODUCTION}

The earthquake information in this publication supplements that published by the United States Geological Survey (USGS) in the National Earthquake Information Service (NEIS) publications, PDE ("Preliminary Determination of Epicenters") and "Preliminary Determination of Epicenters Monthly Listing," by providing detailed felt and intensity data for United States earthquakes. The purpose of this circular is to provide a complete listing of macroseismic effects of earthquakes, which can be used in risk studies, nuclear powerplant site evaluations, seismicity studies, and to answer inquiries by the public. This circular will be the last quarterly issue containing information on United States earthquakes. Beginning with 1983, the data will be published annually in a U.S. Geological Survey special publication entitled "United States Earthquakes".

This publication contains two major sections. The first section (table 1), which is mainly concerned with data obtained by seismographs, is a tabular listing of earthquakes in chronological order by State, consisting of the following basic information: date, origin time, hypocenter, magnitude, maximum intensity, and computational source of the hypocenter. The second section, which concerns intensity information, consists of isoseismal or intensity maps and table 2. This section may contain information about events that were felt but were not listed in the PDE because there was not enough instrumental data to obtain a solution. The list of earthquakes in table 1 was compiled from those located in the United States or nearby offshore areas that were published in the PDE; from aftershock studies carried out by the U.S. Geological Survey and other organizations; from hypocenters in California greater than magnitude 3.0 supplied by the California Institute of Technology, Pasadena, the University of California, Berkeley, and other offices of the U.S. Geological Survey; from hypocenters in Hawaii supplied by the U.S. Geological Survey, Hawaiian Volcano observatory; and from other institutions as listed in the acknowledgments. Known or suspected explosions are also listed in tables 1 and 2 .
The intensities and macroseismic data were compiled from information obtained from postal questionnaires (form shown as fig. 1), from newspaper articles, and from other Government agencies, State institutions, local organizations, and individuals. (See "Acknowledgments" for a list of collaborators.) Figure 1 is the questionnaire currently in use by the USGS. other types of questionnaires are used by state agencies, engineering firms, and other Government agencies to collect intensity data. Anyone wishing to submit felt or damage information on earthquakes for inclusion in future reports should send it to the U.S. Geological Survey, United States Earthquakes Project, Mail Stop 967, Box 25046, Denver Federa1 Center, Denver, CO 80225. Copies of the current "Earthquake Report" questionnaire can be obtained at that address.

The USGS uses the postal questionnaire as the primary source of macroseismic data for an intensity survey; however, on-site field investigations are made following earthquakes that do significant damage. The "Earthquake Report" questionnaires (fig. 1) are mailed to postmasters within the area affected by the earthquake. The completed forms are returned to the USGS, where they are evaluated and intensity values are assigned to individual locations. For large or significant earthquakes, the intensity observations are plotted and isoseismal maps are prepared. Note that the isoseismals represent a general intensity level and that they do not necessarily agree with every individual observation.

\section{DISCUSSION OF TABLES}

The parameters for the earthquakes in tables 1 and 2 include the date, origin time, hypocenter (epicenter and focal depth), magnitude, intensity, and source of the computed solution. The origin time and date are listed in Universal Coordinated Time (UTC) and in local standard time based on the time-zone maps in figures 2 and 3 . The epicenters, which were taken from those published in the PDE or from other sources as noted, are listed here to two decimals. The accuracy of the epicenters is not 
Please enswer this questionnaire and return as soon as possible

1. Was an earthquake felt by anyone in your town near the date and time indicated on the opposite page?
$\square$ No:
Please refold and tepe for return mail.

$\square$ Yes: Date Time

$\square$ AM $\square$ Standard time
$\square$ PI: $\square$ Daylight time

Name of person filling out form

Address

City__._._._. County

State_ Zip code

If you felt the earthquake, complete the following section. If others felt the ear thquake but you did not, skip the personal report and complete the community report.

\section{PERSONAL REPORT}

2. Did you personally feel the earthquake? $1 \square$ Yes $\square$ No Were you awakened by the earthquake? $2 \square$ Yes $\square$ No Were you frightened by the earthquake? $3 \square$ Yes $\square$ No
were you at
4 Home
$5 \square$ Work
$6 \square$ Other?

Town and zip code of your location at time of earthquake

Check your activity when the earthquake occurred:

$7[$ Walking

$11 \square$ Driving (car in motion)

were you

$12 \square$ Sitting

$14 \square$ Inside or

$9 \square$ Lying down

$10 \square$ Standing

If inside, on what floor were you?

16

$13 \square$ Other

150 Outside?

Did you have difficulty in standing or walking $17 \square$ Yes $18 \square$ No

Vibration could be described as $19 \square$ Light $20 \square$ Moderate $21 \square$ Strong
Was there earth noise?
Direction of noise
$\square$ No
$22 \square$ Faint
$\square$ North
$\square$ South
23 $\sqcup$ Moderate
$\square$ East
$24 \square$ Loud
$\square$ West

Estimated duration of

$25 \square$ Sudden, sharp (less than 10 secs)

$26 \square$ Long (30-60 secs)

shaking

$27 \square$ short (10-30 secs)

Continue on to next section which should include personal as wall as raported observations.

\section{COMMUNITY REPORT}

Town and zip code

DO NOT INCLUDE EFFECTS FROM OTHER COMMUNITIES/TOWNS

Check one box for each question that is applicable.

\begin{tabular}{rllll}
\hline 3a. The earthquake was felt by $\square$ No one $28 \square$ Few & $29 \square$ Several & $30 \square$ Many & $31 \square$ All? \\
b. This earthquake awakened $\square$ No one $32 \square]$ Few & $33 \square$ Several & $34 \square$ Many & $35 \square$ All? \\
c. This earthquake frightened $\square$ No one $36 \square$ Few & $37 \square$ Several & $38 \square$ Many & $39-$ All?
\end{tabular}

c. This earthquake frightened $\square$ No one $36 \square$ Few

$37 \square$ Several

$34 \square$ Many $35 \square$ All?

4. What indoor physical effects were noted in your community?

$\begin{array}{lcc}\text { Windows, doors, dishes rattled } & 40 \square \text { Yes } & \square \text { No } \\ \text { Walls creaked } & 41 \square \text { Yes } & \square \text { No } \\ \text { Building trembled (shook) } & 42 \square \text { Slightly } & 43 \square \text { Strongly } \\ \text { Hanging pictures (more than one) } & 44 \square \text { Swung } 45 \square \text { Out of place } 46 \square \text { Fallen } \\ \text { Windows } 4 / \square \text { Few cracked } & 48.1 \text { Some broken out } 49 \square \text { Many broken out } \\ \text { Small objects overturned } & 50 \square \text { Few } & 51 \square \text { Many } \\ \text { Small objects fallen } & 52 \square \mathrm{Few} & 53 \square \text { Many } \\ \text { Glassware/dishes broken } & 54 \square \text { Few } & 55 \square \text { Many } \\ \text { Light furniture or small appliances } & 56 \square \text { Overturned } 57 \square \text { Damaged seriously } \\ \text { Heavy furniture or appliances } & 58 \square \text { Overturned } 59 \square \text { Damaged seriously } \\ \text { Did hanging objectsordoors swing? } & 60 \square \text { sligh }\end{array}$

Did hanging objectsordoors swing? $60 \square$ slightly

Can you estimate direction? $[$ North/South $\square$ East/West $\square$ Other

Items thrown from store shelves $\quad 63 \square$ Few $\quad 64 \square$ Many

Continued on the reverse side

FIGURE 1.--Example of the "Earthquake Report" form used for evaluating the intensities of earthquakes. A, front side. 
5. Indicate effects of the following types to interior walis if any:

Plaster/stucco $65 \square$ Hairline cracks $66 \square$ Large cracks (many) $67[$ Fell in large amounts

Dry well $\quad 68 \square$ Hairline cracks $69 \square$ Large cracks (many) $70 \square \mathrm{Fell}$ in large amounts

6. What outdoor physical effects were noted in your community?

\begin{tabular}{|c|c|c|c|}
\hline $\begin{array}{l}\text { Trees and bushes shaken } \\
\text { Standing vehicles rocked } \\
\text { Moving vehicles rocked }\end{array}$ & $\begin{array}{l}71 \square \text { Slightly } \\
74 \square \text { Slightly } \\
76 \square \text { Slightly }\end{array}$ & $\begin{array}{l}72 \square \text { Moderately } \\
75 \square \text { Moderately } \\
77 \square \text { Moderately }\end{array}$ & $73 \square$ Strongly \\
\hline $\begin{array}{l}\text { Water splashed onto sides of } \\
\text { lakes, ponds, swimming pools }\end{array}$ & $78 \square$ Yes & $\square$ No & \\
\hline Elevated water tanks & $79 \square$ Cracked & $80 \square$ Twisted & $\begin{array}{l}81 \square \text { Fallen } \\
\text { (thrown down) }\end{array}$ \\
\hline Tombstones & $\begin{array}{l}82 \square \text { Displaced } \\
85 \square \text { Fallen }\end{array}$ & $83 \square$ Cracked & $84 \square$ Rotated \\
\hline Chimneys & $\begin{array}{l}86 \square \text { Cracked } \\
89 \square \text { Broken at roof }\end{array}$ & f line $\begin{array}{r}87 \square \text { Twisted } \\
90 \square \mathrm{B}\end{array}$ & $\begin{array}{l}88 \square \text { Fallen } \\
\text { ricks fallen }\end{array}$ \\
\hline $\begin{array}{l}\text { Railroad tracks bent } \\
\text { Stone or brick fences/walls }\end{array}$ & $\begin{array}{l}91 \square \text { Slightly } \\
93 \square \text { Open cracks }\end{array}$ & $\begin{array}{l}92 \square \text { Greatly } \\
94 \square \text { Fallen }\end{array}$ & $95 \square$ Destroyed \\
\hline Underground pipes & $96 \square$ Broken & $97 \square$ Out of service & \\
\hline $\begin{array}{l}\text { Highways or streets } \\
\text { Sidewalks }\end{array}$ & $\begin{array}{l}98 \square \text { Large cracks } \\
100 \square \text { Large cracks }\end{array}$ & $\begin{array}{l}99 \square \text { Large displa } \\
101 \square \text { Large displa }\end{array}$ & $\begin{array}{l}\text { cements } \\
\text { cements }\end{array}$ \\
\hline
\end{tabular}

7a. Check below any structural damage to buildings.
Foundation $102 \square$ Cracked
Interior walls
$104 \square$ Split $105 \square$ Fallen
$103 \square$ Destroyed
Exterior walls
$107 \square$ Large Cracks
$106 \square$ Separated from ceiling or floor
109 [ Partial collapse
108 [ Bulged outward
$110 \square$ Total collapse

b. What type of construction was the building that showed this damage?
$111 \square$ wood
$112 \square$ Stone
$113 \square$ Brick veneer
$114 \square$ Other
$115 \square$ Brick
$116 \square$ Cinderblock
$117 \square$ Reinforced concrete
$118 \square$ Mobile home

c. What was the type of ground under the building?
$\square$ Don't know
$119 \square$ Sandy soil
if $3 \square$ Clay soil
$120 \square$ Marshy
$121 \square$ Fill
$122 \square$ Hard rock
12477 Sandstone, limestone, shale

d. Was the ground:

$125 \square$ Level

$126 \square$ sloping

$127 \square$ Steep?

e. Check the approximate age of the building:
$128 \square$ Built before 1935
$129 \square$ Built 1935-65
$130 \square$ Built after 1965

8. Check below any structural damage to

$\begin{array}{llll}\text { Bridges/Overpasses } & 131 \square \text { Concreta } & 132 \square \text { Wood } 133 \square \text { Steel } & 134 \square \text { Other } \\ \text { Damage was } & 135 \square \text { Slight } & 136 \square \text { Moderate } & 137 \square \text { Severe } \\ \text { Dams } & 138 \square \text { Concrete } & 139 \square \text { Large earthen } & \\ \text { Damage was } & 140 \square \text { Slight } & 141 \square \text { Moderate } & 142 \square \text { Severe }\end{array}$

9. What geologic effects were noted in vour community?

$\begin{array}{lccc}\text { Ground cracks } & 143 \square \text { Wet ground } & 144 \square \text { Steep slopes } & 145 \square \text { Dry and level } \\ \text { Landslides } & 146 \square \text { Small } & 147 \square \text { Large } & \text { ground } \\ \text { Slumping } & 148 \square \text { River bank } & 149 \square \text { Road fill } & 150 \square \text { Land fill } \\ \text { Were springs or well water disturbed? } & 151 \quad \text { Level changed } & 152 \square \text { Flow disturbed } \\ & 153 \quad \text { Muddied } & \square \text { Don't know } \\ \text { Were rivers or lakes changed? } & 154 \text { Yes } & \square \text { No } & \square \text { Don't know }\end{array}$

10a. What percentage of buildings were damaged?

Within 2 city blocks of your location

None
156 Many (about $50 \%$ )
$159-$ Mane
Many (about $50 \%$ )

$155 \square$ Few (about $5 \%$ )

$157 \square$ Most (about $75 \%$ )

$158 \square$ Few (about 5\%)

b. In area covered by your zip code

$159-$ Many (about 50\%) $160 \square$ Most (about $75 \%$ )

Thank you for your time and information. Refold this card and tape for return mall.

FIGURE 1.--Example of the "Earthquake Report" form used for evaluating the intensities of earthquakes. $\underline{B}$, reverse side. 


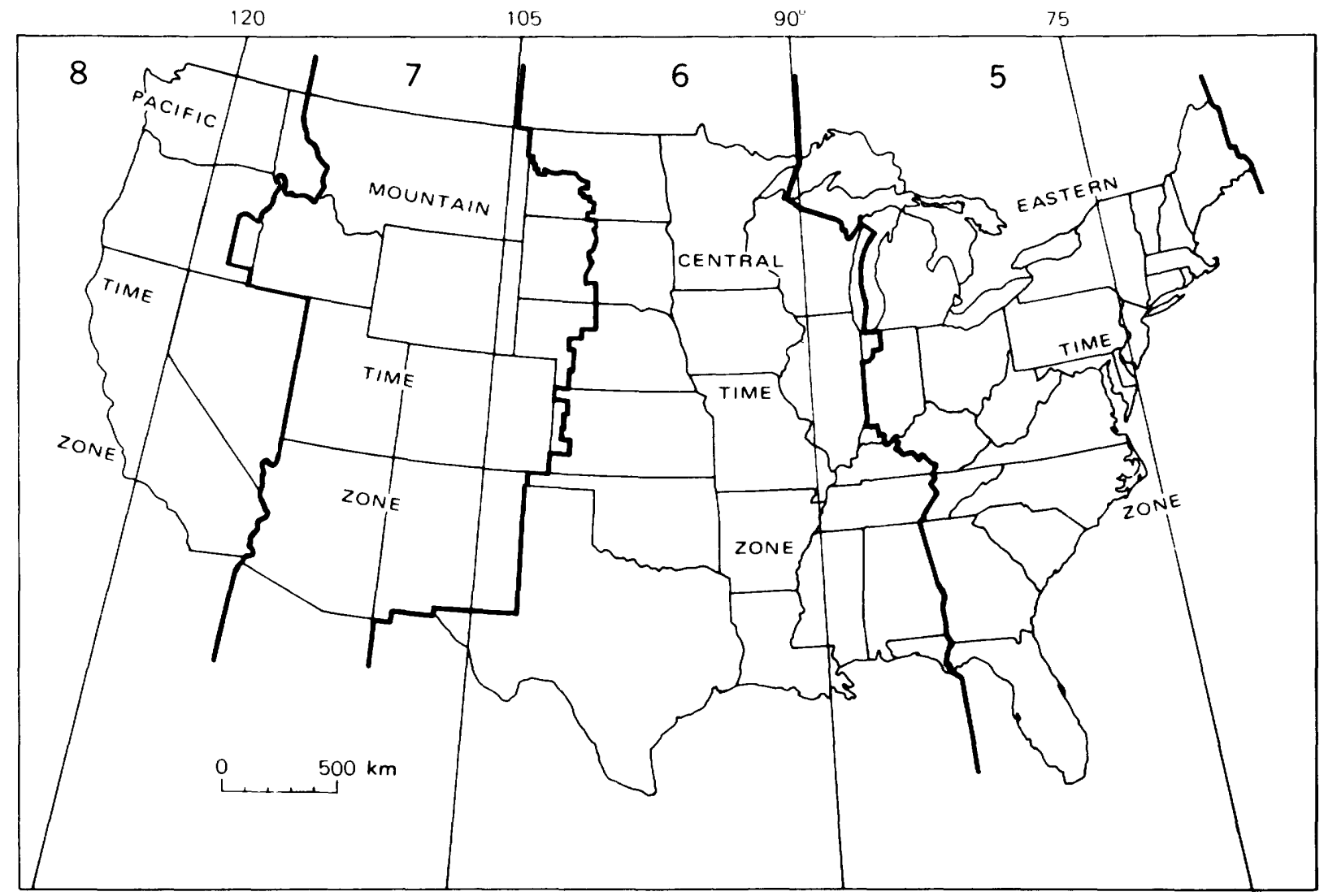

FIGURE 2.--Standard time zones of the conterminous United States. The number in each zone shows the number of hours to be subtracted from Universal Coordinated Time to convert to local standard time. (Subtract 1 hour less for local daylight-saving time.)

necessarily indicated by the number of decimals listed. The epicenters located by the NEIS usually are accurate to two-tenths of a degree or less. In general, epicenters located offshore are less accurate than those on land, even though they are listed to two decimals. In regions covered by dense networks of seismo-

graphs, such as California, epicenter accuracy is significantly better than the two-tenths of a degree listed. Depths are listed to the nearest whole kilometer.

The magnitude values listed in tables 1 and 2 were furnished by cooperating institutions or determined by USGS. The computational sources are labeled according to the assigned letter codes shown in headnotes to tables 1 and 2; the letter follows the value listed under the column heading "Magnitude." In table 1, the absence of a letter code indicates that the source is USGS. The magnitude values calculated by USGS are based on the following formulas: as adopted by the International Association of Selsmology and Physics of the Earth's Interior (IASPEI; Bath, 1966, p. 153), where A is the maximum vertical surface-wave ground amplitude, in micrometers; $T$ is the period, in seconds, and $18<T<22$; and $D$ is the distance, in geocentric degrees (station to epicenter), and $20^{\circ} \leq D \leq 160^{\circ}$. No depth correction is made for depths less than $50 \mathrm{~km}$.

$$
\mathrm{mb}=\log (\mathrm{A} / \mathrm{T})+\mathrm{Q}(\mathrm{D}, \mathrm{h}),
$$

as defined by Gutenberg and Richter (1956), except that $T$, the period in seconds, is restricted to $0.1<\mathrm{T}<3.0$, and $A$, the ground amplitude in micrometers, is not necessarily the maximum of the $P$-wave group. $Q$ is a function of distance $D$ and depth $h$, where $D \geq 5^{\circ}$.

$$
M L=\log A-\log A,
$$

as defined by Richter (1958, p. 340), where A is the maximum trace amplitude in millimeters, written by a Wood-Anderson torsion seismometer, and $\log A$ is a standard value as a function of distance, where the distance is $\leq 600 \mathrm{~km}$. ML 


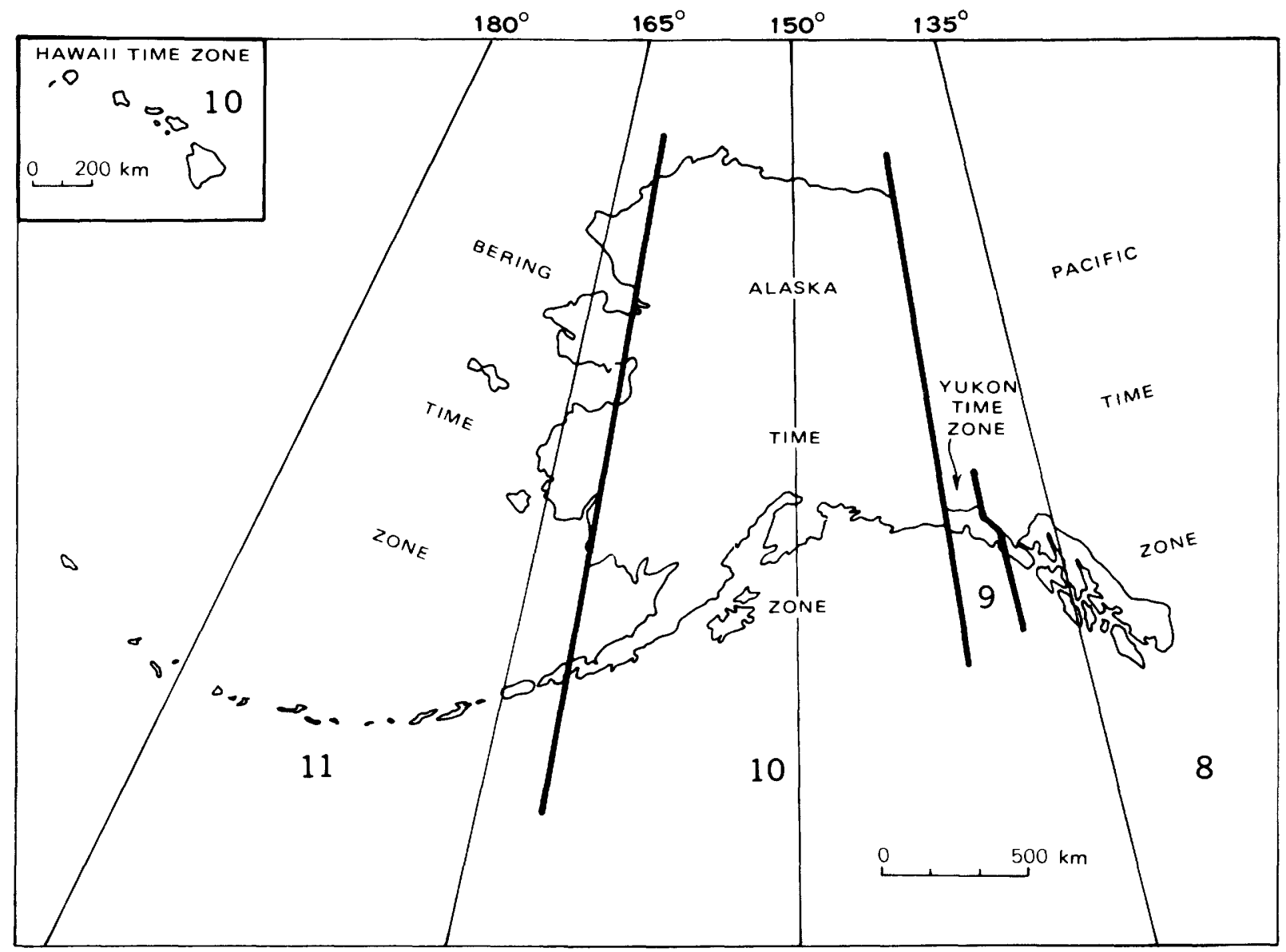

FIGURE 2.--Standard time zones of the conterminous United States. The number in each zone shows the number of hours to be subtracted from Universal Coordinated Time to convert to local standard time. (Subtract 1 hour less for local daylight-saving time.)

values are also calculated from other seismometers by conversion of recorded ground motion to the expected response of the torsion seismometer.

$$
\begin{gathered}
M n=3.75+0.90(\log D)+\log (A / T) \\
0.5^{\circ} \leq D \leq 4^{\circ}, \\
M n=3.30+1.66(\log D)+\log (A / T) \\
4^{\circ} \leq D \leq 30^{\circ},
\end{gathered}
$$

as proposed by Nutt1i (1973), where $A / T$ is expressed in micrometers per second, calculated from the vertical-component 1 -second $\mathrm{Lg}$ waves, and $\mathrm{D}$ is the distance in geocentric degrees.

MD is used in this publication for the duration or coda length magnitude. MD is usually computed from the difference, in seconds, between Pn- or Pg-wave arrival time and the time the final coda amplitude decreases to the background-noise amplitude. These magnitudes are normally correlated with ML or mbLg so that resulting magnitudes are compatible. Thus the formulas vary for different geographic regions and seismograph systems.

Figures 4-6 are maps summarizing the earthquake activity for the conterminous United States, Alaska, and Hawail for the period October-December 1982. The magnitudes represented in these figures are based on $M L$, $\mathrm{Mn}$, or $\mathrm{MD}$; if none of these were computed, then on MS; and finally on $\mathrm{mb}$, when it was the only magnitude computed.

All the intensity values (indicated by Roman numerals) listed in this summary were determined, using the Modified Mercalli Intensity Scale of 1931 (Wood and Neumann, 1931) shown below, from the evaluation of "Earthquake Report" forms; from field reports by U.S. Geological Survey personnel, engineering firms, or universities; and from detailed macroseismic data communicated to the USGS by people in the area affected by the earthquake. All earthquake reports received that contain minimal or sketchy information are listed only as "FELT". This 


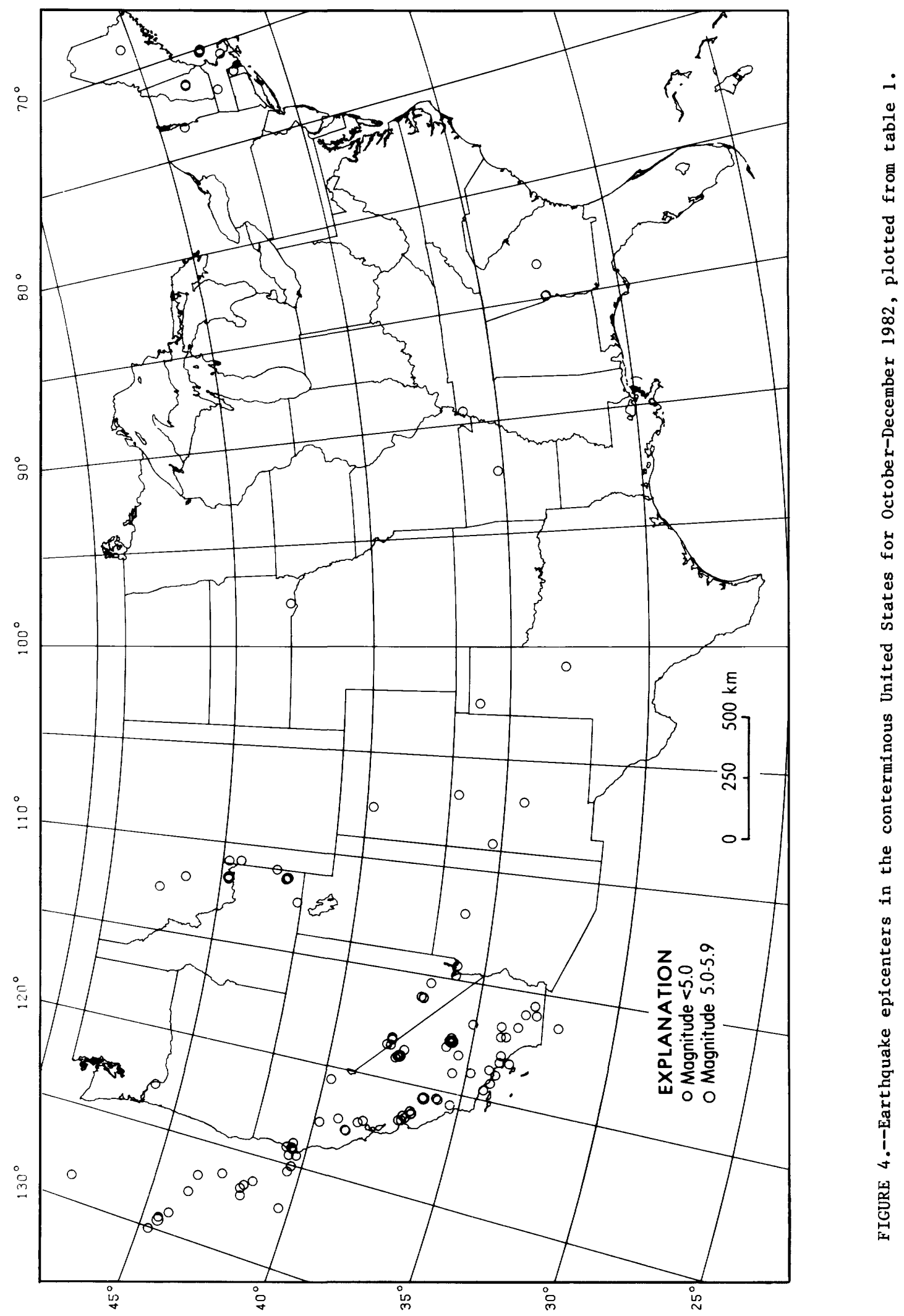




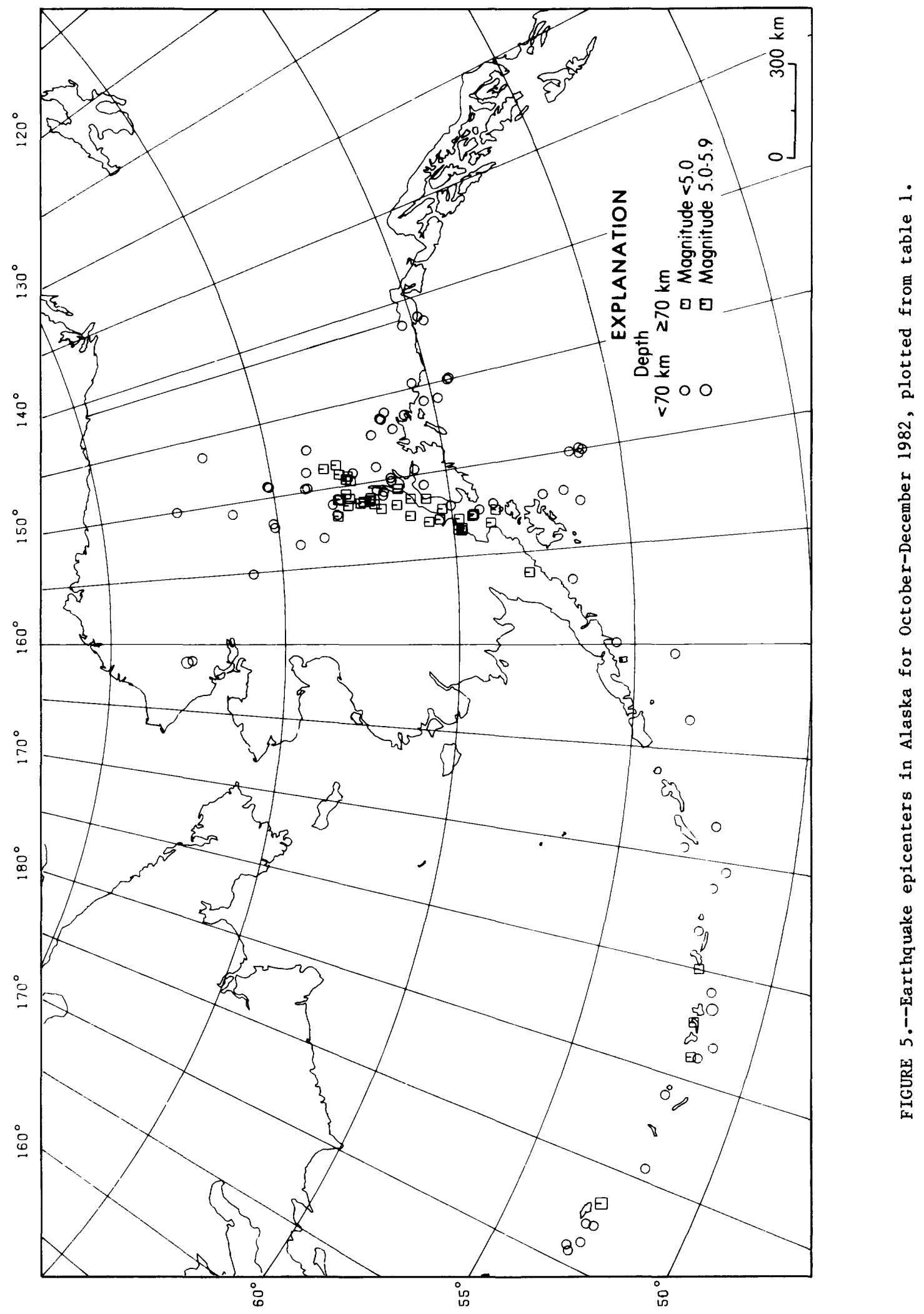




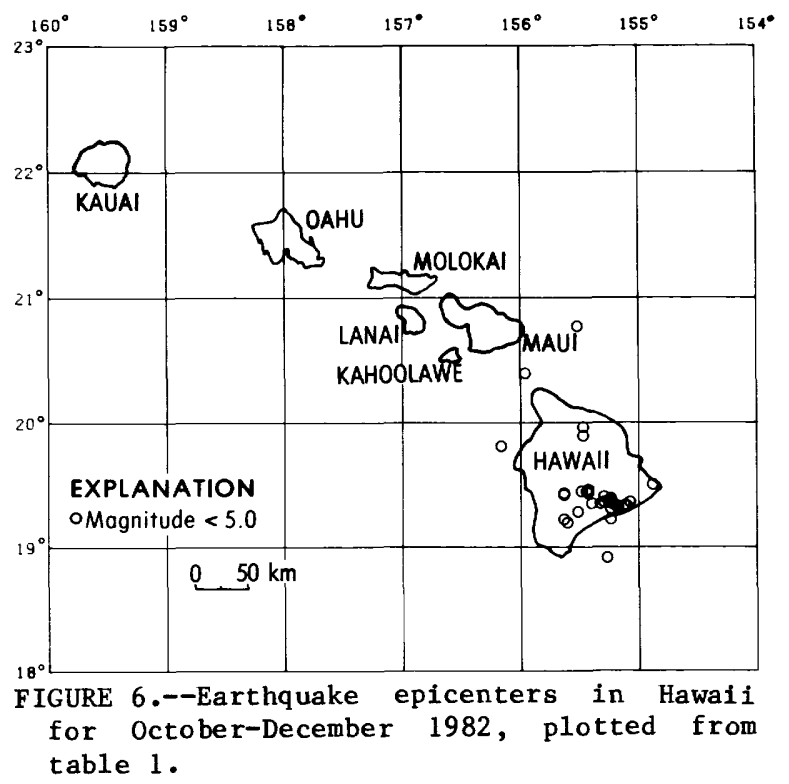

designation does not imply that the earthquake was felt at a low intensity level, but indicates that the available data are not sufficient for assigning a valid intensity value. These reports are filed in the offices of the NEIS or in government archives and are available for detailed study.

\section{MODIFIED MERCALLI INTENSITY SCALE OF 1931}

Adapted from Sieberg's Mercalli-Cancani scale, modified and condensed.

I. Not felt - or, except rarely under especially favorable circumstances. Under certain conditions, at and outside the boundary of the area in which a great shock is felt: sometimes birds, animals, reported uneasy or disturbed; sometimes dizziness or nausea experienced; sometimes trees, structures, liquids, bodies of water, may sway--doors may swing, very slowly.

II. Felt indoors by few, especially on upper floors, or by sensitive, or nervous persons. Also, as in grade I, but often more noticeably: sometimes hanging objects may swing, especially when delicately suspended; sometimes trees, structures, liquids, bodies of water, may sway, doors may swing, very slowly; sometimes birds, animals, reported uneasy or disturbed; sometimes dizziness or nausea experienced.

III. Felt indoors by several, motion usually rapid vibration. Sometimes not recognized to be an earthquake at first. Duration estimated in some cases. Vibra- tion like that due to passing of light, or lightly loaded trucks, or heavy trucks some distance away. Hanging objects may swing slightly. Movements may be appreciable on upper levels of tall structures. Rocked standing motor cars slightly.

IV. Felt indoors by many, outdoors by few. Awakened few, especially light sleepers. Frightened no one, unless apprehensive from previous experience. Vibration like that due to passing of heavy or heavily loaded trucks. Sensation like heavy body striking building or falling of heavy objects inside. Rattling of dishes, windows, doors; glassware and crockery clink and clash. Creaking of walls, frame, especially in the upper range of this grade. Hanging objects swung, in numerous instances. Disturbed liquids in open vessels slightly. Rocked standing motor cars noticeably.

V. Felt indoors by practically all, outdoors by many or most: outdoors direction estimated. Awakened many, or most. Frightened few-slight excitement, a few ran outdoors. Buildings trembled throughout. Broke dishes, glassware, to some extent. Cracked windows--in some cases, but not generally. Overturned vases, small or unstable objects, in many instances, with occasional fall. Hanging objects, doors, swing generally or considerably. Knocked pictures against walls, or swung them out of place. Opened, or closed, doors, shutters, abruptly. Pendulum clocks stopped, started or ran fast, or slow. Moved small objects, furnishings, the latter to slight extent. Spilled liquids in small amounts from we11-filled open containers. Trees, bushes, shaken slightly.

VI. Felt by all, indoors and outdoors. Frightened many, excitement general, some alarn, many ran outdoors. Awakened a11. Persons made to move unsteadily. Trees, bushes, shaken slightly to moderately. Liquid set in strong motion. Small bells rang--church, chape1, school, etc. Damage slight in poorly built buildings. Fall of plaster in small amount. Cracked plaster somewhat, especially fine cracks chimneys in some instances. Broke dishes, glassware, in considerable quantity, also some windows. Fall of knickknacks, books, pictures. Overturned furniture in many instances. Moved furnishings of moderately heavy kind.

VII. Frightened all--general alarm, all ran outdoors. Some, or many, found it difficult to stand. Noticed by persons driving motor cars. Trees and bushes shaken 
moderately to strongly. Waves on ponds, lakes, and running water. Water turbid from mud stirred up. Incaving to some extent of sand or gravel stream banks. Rang large church bells, etc. Suspended objects made to quiver. Damage negligible in buildings of good design and construction, slight to moderate in wellbuilt ordinary buildings, considerable in poorly built or badly designed buildings, adobe houses, old walls (especially where laid up without mortar), spires, etc. Cracked chimneys to considerable extent, walls to some extent. Fall of plaster in considerable to large amount, also some stucco. Broke numerous windows, furniture to some extent. shook down loosened brickwork and tiles. Broke weak chimneys at the roof-line (sometimes damaging roofs). Fall of cornices from towers and high buildings. Dislodged bricks and stones. Overturned heavy furniture, with damage from breaking. Damage considerable to concrete irrigation ditches.

VIII. Fright general--alarm approaches panic. Disturbed persons driving motor cars. Trees shaken strongly--branches, trunks, broken off, especially palm trees. Ejected sand and mud in small amounts. Changes: temporary, permanent; in flow of springs and wells; dry wells renewed flow; in temperature of spring and well waters. Damage slight in structures (brick) built especially to withstand earthquakes. Considerable in ordinary substantial buildings, partial collapse: racked, tumbled down, wooden houses in some cases; threw out panel walls in frame structures, broke of $f$ decayed piling. Fall of walls. Cracked, broke, solid stone walls seriously. Wet ground to some extent, including ground on steep slopes. Twisting, fall, of chimneys, columns, monuments, also factory stacks, towers. Moved conspicuously, overturned, very heavy furniture.

IX. Panic general. Cracked ground conspicuously. Damage considerable in (masonry) structures built especially to withstand earthquakes: Threw out of plumb some wood-frame houses built especially to withstand earthquakes; great in substantial (masonry) buildings, some collapse in large part; or wholly shifted frame buildings off foundations, racked frames; serious to reservoirs; underground pipes sometimes broken.
X. Cracked ground, especially when loose and wet, up to widths of several inches; fissures up to a yard in width ran parallel to canal and stream banks. Landslides considerable from river banks and steep coasts. Shifted sand and mud horizontally on beaches and flat land. Changed level of water in wells. Threw water on banks of canals, lakes, rivers, etc. Damage serious to dams, dikes, embankments. Severe to well-built wooden structures and bridges, some destroyed. Developed dangerous cracks in excellent brick walls. Destroyed most masonry and frame structures, also their foundations. Bent railroad rails slightly. Tore apart, or crushed endwise, pipe lines buried in earth. Open cracks and broad wavy folds in cement pavements and aspha1t road surfaces.

XI. Disturbances in ground many and widespread, varying with ground material. Broad fissures, earth slumps, and 1 and slips in soft, wet ground. Ejected water in large amounts charged with sand and mud. Caused sea-waves ("tidal" waves) of significant magnitude. Damage severe to wood-frame structures, especially near shock centers. Great to dams, dikes, embankments often for long distances. Few, if any (masonry) structures remained standing. Destroyed large well-built bridges by the wrecking of supporting piers, or pillars. Affected yielding wooden bridges less. Bent railroad rails greatly, and thrust them endwise. Put pipe lines buried in earth completely out of service.

XII. Damage total--practically all works of construction damaged greatly or destroyed. Disturbances in ground great and varied, numerous shearing cracks. Landslides, falls of rock of significant character, slumping of river banks, etc., numerous and extensive. Wrenched loose, tore off, large rock masses. Fault slips in firm rock, with notable horizontal and vertical offset displacements. Water channels, surface and underground, disturbed and modified greatly. Dammed lakes, produced waterfalls, deflected rivers, etc. Waves seen on ground surfaces (actually seen, probably, in some cases). Distorted lines of sight and level. Threw objects upward into the air. 
Table 1.-Summary of U. S. earthquakes for October-December 1982

(Sources of the hypocenters and magnitudes: (B) University of California, Berkeley; (C) Los Alamos Natínal Laboratory, New Mexico; (E) U.S. Department of Energy, Las Vegas, Nev,; (F) Georgia Institute of Technology, Atlanta; (G) U.S. Geological Survey, Golden, Colo, and Menlo Park, Calif.; (H) U.S. Geological Survey, Hawaiian Volcano Observatory; (J) Weston Observatory, Mass.; (K) Tennessee Earthquake Information Center,
Memphis; (L) Lamont-Uoherty Geological Observatory, Palisades, N.Y.; (M) National Oceanic and Atmospheric Administration, Alaska Tsunami Warning Center, Palmer; (P) California Institute of Technology, Pasadena; (T) Oklahoma Geological Survey, Leonard; (U) University of Utah, Salt Lake City; N, Normal depth; UTC, Universal Coordinated Time. For names of local time zones, see figures 2 and 3 . Leaders $(\ldots)$ indicate no information availablej

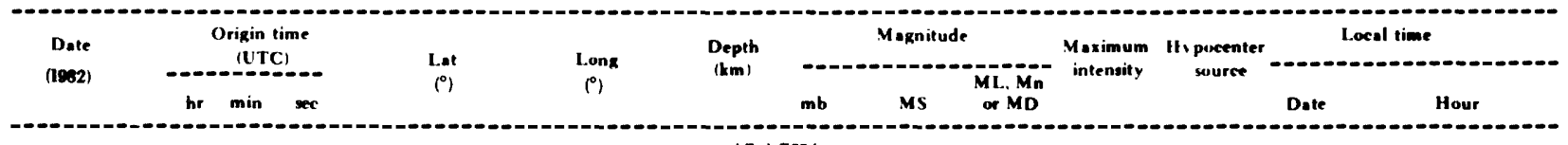

ALASKA

OCT. $1 \quad 07 \quad 59 \quad 14.0$

OCT: 1144255.1

$\begin{array}{lllll}\text { OCT: } & 1 & 15 & 35 & 48.8 \\ \text { OCT: } & 2 & 04 & 38 & 04.9\end{array}$

OCT. $2 \quad 111407.2$

$\begin{array}{lllll}\text { OCT. } & 3 & 16 & 18 & 10.6 \\ \text { OCT. } & 4 & 07 & 46 & 52.8\end{array}$

OCT. $\quad 5 \quad 172035.8$

$\begin{array}{lrrrrr}\text { OCT. } & 8 & 10 & 44 & 52.5 \\ \text { OCT. } & 11 & 15 & 31 & 12.1\end{array}$

OCT. $12 \quad 08 \quad 47 \quad 14.3$

$\begin{array}{lllll}\text { OCT: } & 14 & 04 & 47 & 12.2 \\ \text { OCT. } & 15 & 15 & 19 & 20.1\end{array}$

OCT: $15 \quad 193129: 1$

OCT. $16 \quad 092248.5$

$\begin{array}{lllll}\text { OCT. } & 17 & 18 & 50 & 00.3 \\ \text { OCT. } & 18 & 04 & 16 & 34.7\end{array}$

OCT. $18 \quad 121910.5$

$\begin{array}{lllll}\text { OCT. } & 19 & 19 & 20 & 08.8 \\ \text { OCT. } & 21 & 11 & 10 & 34.7\end{array}$

OCT. $23 \quad 085127.6$

OCT. $24 \quad 044403.7$

$\begin{array}{lllll}\text { OCT. } & 25 & 13 & 48 & 09.6 \\ \text { OCT. } & 27 & 14 & 32 & 24.7\end{array}$

OCT. $27 \quad 16 \quad 14 \quad 04.7$

$\begin{array}{lllll}\text { OCT. } & 28 & 14 & 41 & 26.5 \\ \text { OCT. } & 30 & 09 & 53 & 13.4\end{array}$

NOV. 2024643.2

NOV: $2 \quad 180019.5$

NOV: $3 \quad 063401.9$

NOV. $400 \quad 04 \quad 56.5$

$\begin{array}{lllll}\text { NOV: } & 4 & 05 & 18 & 41.2 \\ \text { NOV: } & 5 & 03 & 39 & 44.5\end{array}$

NOV: 5 13 0646.7

NOV. $5 \quad 13 \quad 14 \quad 27.3$

$\begin{array}{lllll}\text { NOV } & 6 & 00 & 57 & 47.2 \\ \text { NOV } & 6 & 13 & 01 & 47.7\end{array}$

NOV. $6 \quad 233458.7$

NOV. $\quad 7 \quad 060609.0$

NOV: $8 \quad 143935.6$

NOV. $\quad \begin{array}{lllll}9 & 01 & 01 & 02.4\end{array}$

$\begin{array}{lllll}\text { NOV: } & 9 & 06 & 48 & 44.7 \\ \text { NOV. } & 9 & 18 & 55 & 00.4\end{array}$

NOV. $10 \quad 032237.1$

NOV. $10 \quad 04 \quad 3512.0$

NOV. $\quad 10 \quad 081428.9$

NOV: $10 \quad 1723 \quad 52.5$

NOV. $10 \quad 225515.9$

$\begin{array}{lllll}\text { NOV: } & 12 & 22 & 31 & 54.3 \\ \text { NOV: } & 15 & 01 & 06 & 15.6\end{array}$

NOV. $15 \quad 1916 \quad 54.5$

$\begin{array}{lllll}\text { NOV: } & 16 & 22 & 10 & 12.8 \\ \text { NOV: } & 17 & 05 & 27 & 27.5\end{array}$

NOV: $17 \quad 120149.8$

NOV $\quad \begin{array}{lllll}18 & 00 & 14 & 28.7\end{array}$

$\begin{array}{lllll}\text { NOV. } & 18 & 01 & 21 & 47.2 \\ \text { NOV. } & 19 & 16 & 43 & 56.4\end{array}$

NOV: $21 \quad 001736.7$

NOV. $21 \quad 104807.3$

NOV: $21 \quad 141645.6$
$61.61 \mathrm{~N} .146 .36 \mathrm{~W}$

$56.18 \mathrm{~N}$.

$60.31 \mathrm{~N}$.

$56.30 \mathrm{~N}$.

$146.36 \mathrm{~W}$.
$149.93 \mathrm{~W}$.
$141.40 \mathrm{~W}$.
$163.58 \mathrm{~W}$.
$152.61 \mathrm{~W}$.

64
$33 \mathrm{~N}$

15

$4 \cdot 0$
4.1

...

$\ldots$

$\ddot{3} \dot{9 M}$
$\ddot{3} \ddot{3 M}$

$\ldots$

$\ldots$

$\ldots$

$\begin{array}{ll}\mathrm{G} & \mathrm{SEPT} \\ \mathrm{G} & \mathrm{OCT} \text {. } \\ \mathrm{G} & \text { OCT. }\end{array}$

$G$ OCT:

$56.07 \mathrm{~N}$.

$51.44 \mathrm{~N}$.

$150.02 \mathrm{~W}$.
$176.62 \mathrm{~W}$.

$33 \mathrm{~N} \quad 4.2$

$61.59 \mathrm{~N}$

$150.13 \mathrm{~W}$.

$\begin{array}{ll}38 & 4.2 \\ 60 & .5\end{array}$

$\cdots \quad 3.3 \mathrm{M}$

$152.48 \mathrm{~W} . \quad 33 \mathrm{~N} \quad \ddot{3.8}$

$\ddot{5} \dot{0}$

5. $6 \mathrm{M}$

$61.60 \mathrm{~N}$.

$65.85 \mathrm{~N}$.

$60.48 \mathrm{~N}$.

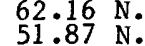

$60.70 \mathrm{~N}$.

$155.10 \mathrm{~W} . \quad 33 \mathrm{~N}$

14.174 W. 15
144.74 W. 106

151.36
177.40
150.65

$150.65 \mathrm{~W} . \quad 33 \mathrm{~N}$

$59.67 \mathrm{~N}$.

$65.10 \mathrm{~N}$.

$141.47 \mathrm{~W}$

$59.46 \mathrm{~N}$.

$173.28 \mathrm{~W}$.

15

$149.33 \mathrm{~W} .15$

$\begin{array}{lll}149.33 & \text { W. } & 15 \\ 174.98 & \text { W. } & 33 \mathrm{~N} \\ 172.13 & \mathrm{E} . & 33 \mathrm{~N}\end{array}$

$52.73 \mathrm{~N}$.

$62.81 \mathrm{~N}$.

$59.40 \mathrm{~N}$.

$56.72 \mathrm{~N}$.
$52.34 \mathrm{~N}$.

$62.86 \mathrm{~N}$.

$149.43 \mathrm{~W} .78$

$\begin{array}{llll}149.43 & \text { W. } & 78 & \\ 144.91 & \mathrm{~W} \cdot & 33 \mathrm{~N} & 4.0 \\ 156.63 & \mathrm{~W} . & 66 & 4.8 \\ 168.43 & \mathrm{~W} . & 33 \mathrm{~N} & 5.1\end{array}$

$150.83 \mathrm{~W} \cdot 112$

$52.16 \mathrm{~N}$.

1

$52.65 \mathrm{~N}$.

$62.56 \mathrm{~N}$.

$171.25 \mathrm{~W} . \quad 33 \mathrm{~N} \quad 4$

$178.93 \mathrm{~W}$. 8

171.26 W. 68 .

$151.20 \mathrm{~W} .115$...

$151.62 \mathrm{~W} .120$

$62.92 \mathrm{~N}$.

$56.17 \mathrm{~N}$.

$67.81 \mathrm{~N}$.

$151.26 \mathrm{~W} .156$

$\begin{array}{lll}150.22 & \mathrm{~W} . & 33 \mathrm{~N} \\ 150.06 & \mathrm{~W} . & 33 \mathrm{~N}\end{array}$

$61.12 \mathrm{~N}$.

$151.35 \mathrm{~W} .100$

$62.74 \mathrm{~N}$.

149.73 W. 97

$62.93 \mathrm{~N}$

$58.80 \mathrm{~N}$.

$151.73 \mathrm{~W} .131$

$150.50 \mathrm{~W} .121$

$65.13 \mathrm{~N}$.

$152.21 \mathrm{~W} \cdot \quad 33 \mathrm{~N}$
$151.79 \mathrm{~W}$.
$33 \mathrm{~N}$

... $3.1 \mathrm{M}$

FËLT

$G$ OCT.

$\frac{1}{1}$

09 P.M. AST

04 A.M. AST

05 A.M. AST

01 P.M. BST

$65.08 \mathrm{~N}$.

$\begin{array}{ll}152.05 \mathrm{~W} . & 33 \mathrm{~N} \\ 151.04 \mathrm{~W} . & 22\end{array}$

$62.29 \mathrm{~N}$.

$57.94 \mathrm{~N}$.

$151.16 \mathrm{~W}: 113$

146.40 W. $33 \mathrm{~N}$

$64.42 \mathrm{~N}$.

$153.42 \mathrm{~W} . \quad 33 \mathrm{~N}$

$60.90 \mathrm{~N}$.

$61.44 \mathrm{~N}$.

$146.42 \mathrm{~W} \cdot 40$

$146.03 \mathrm{~W} .33 \mathrm{~N}$

$58.92 \mathrm{~N}$.
$52.86 \mathrm{~N}$.

$\begin{array}{lll}153.26 & \mathrm{~W} & 94 \\ 170.65 & \mathrm{E} & 33 \mathrm{~N}\end{array}$

$63.47 \mathrm{~N}$.

$60.36 \mathrm{~N}$

$148.73 \mathrm{~W} .102$

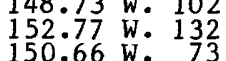

$53.15 \mathrm{~N}$.

$59.85 \mathrm{~N}$.

169.57 W. $33 \mathrm{~N}$

$145.90 \mathrm{~W} . \quad 33 \mathrm{~N}$

$60.26 \mathrm{~N}$.

$58.74 \mathrm{~N}$

$62.46 \mathrm{~N}$

$145.88 \mathrm{~W} \cdot 33 \mathrm{~N}$

152.59 W. 90

$151.15 \mathrm{~W} .106$

149.59 W. 100

$3.2 \mathrm{M}$

$\ldots$

$\begin{array}{ll}\mathrm{G} & \text { OCT. } \\ \mathrm{G} & \text { OCT: } \\ \mathrm{G} & \text { OCT. } \\ \mathrm{OCT}\end{array}$

$\ldots \quad 3.4 \mathrm{M}$

06 A.M. AST

08 P.M. BST

07 A.M. AST

00 A.M. AST

1105 A.M. AST

$62.88 \mathrm{~N}$. $\therefore$

4.3

$\ddot{4} \cdot \dot{2}$

$\ddot{0} 0$

$3.6 \mathrm{M}$

$\because \ldots \quad G$ OCT.

$\ldots$

$\therefore \quad G$ OCT. 15

$\cdots$

.

$\ddot{3} \ddot{\circ}$

-..

OCT

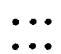$$
\stackrel{G}{G}
$$

$\begin{array}{ll}\text { OCT. } & 17 \\ \text { OCT. } & 17 \\ \text { OCT. } & 18 \\ \text { OCT. } & 19\end{array}$

10 P.M. AST

06 P.M. AST

05 A.M. AST

11 A.M. BST

08 A.M. AST

05 P.M. BST

02 A.M. AST

09 A.M. AST

DO A.M. BST

$\begin{array}{lllllll}\ldots & \ldots & \ldots & \text { G OCT. } 22 & 10 & \text { P.M. AST }\end{array}$

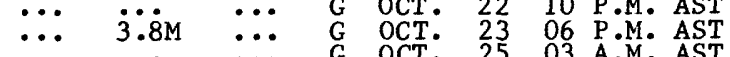

$\begin{array}{llll}\ldots & \ldots & \cdots & \text { G OCT. } 27 \text { O }\end{array}$

$\begin{array}{llll}\ldots & 4.6 \mathrm{M} \quad \ldots \quad \mathrm{G} \text { OCT. } 28\end{array}$

$\because \quad \because \cdots \quad \because \cdots \quad G$ G OCT.

$\because \cdots \quad \because \cdots \quad$ G NOV.

NOV.

03 A.M. BST

10 P.M. BST

04 P.M. AST

07 A.M. BST

$\ldots \quad \ldots$ FELT G NOV.

$\because \quad \because \cdots \quad 4.2 \mathrm{M} \quad \because \cdots \quad$ G NOV.

$\because \quad \because . \quad 4.5 \mathrm{M}$ FELT G NOV.

02 P.M. AST

307 P.M. AST

$\begin{array}{lll}4 & 05 & \text { P.M. AST } \\ 5 & 03 & \text { A.M. AST }\end{array}$

03 A.M. AST

$3.9 \quad \ldots \quad \quad \cdots \quad \quad \ldots \quad$ G NOV.

5 02 P.M. AST

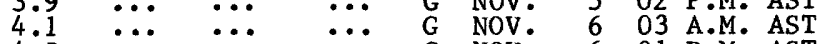

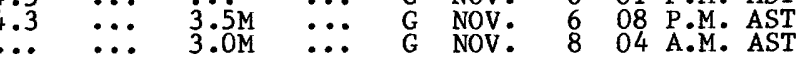

$\begin{array}{llllll}\ldots & \ldots & 2.8 \mathrm{M} \quad \ldots & \text { G NOV. } 8 & 03 & \text { P.M. AST }\end{array}$

$\ldots \quad \ldots \quad 2.5 M \quad \ldots \quad$ G NOV. 8 O 08 P.M. AST

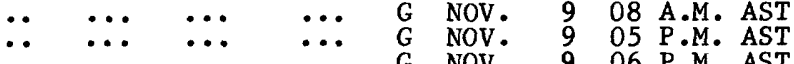

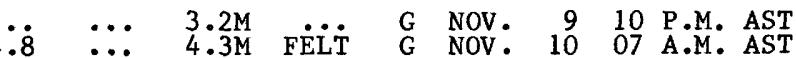

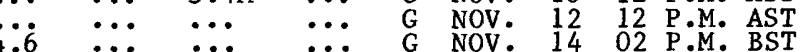

$3.9 \quad \ldots$ G NOV. 15 G 09 A.M. AST

$\begin{array}{llllllll} & \cdots & \cdots & \cdots & \cdots & \text { G NOV. } 16 & 12 & \text { P.M. AST }\end{array}$

$\begin{array}{llll}\cdots & \ldots & \ldots & \ldots\end{array} \quad$ G NOV. 16 07 P.M. AST

$\begin{array}{lllllllll}.4 & \ldots & \ldots & \ldots & \text { G NOV. } 17 & 01 & \text { A.M. BST }\end{array}$

.. $\quad \ldots .3 .3 \mathrm{M} \quad \ldots \quad$ G NOV. 17 02 P.M. AST

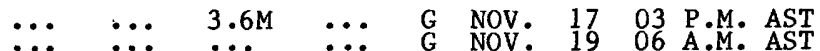

$\because \quad \because \cdots \quad \because \cdots \quad$ G NOV. 19 G 02 A.M. AST

$\begin{array}{llll}\cdots & \cdots & \cdots & \cdots\end{array}$ G NOV. 21 00 A.M. AST

$\because \cdots \quad \cdots \cdots \quad \cdots \cdots \quad \cdots \quad$ G NOV: 21 04 A.M. AST

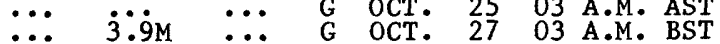

$4.5 \quad \ldots . \quad \ldots . \quad \ldots \quad$ G NOV. 6 o1 P.M. AST

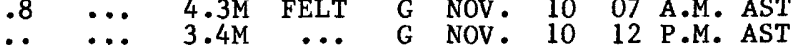


Table 1.--Summary of U. S. earthquakes for October-December 1982--Continued

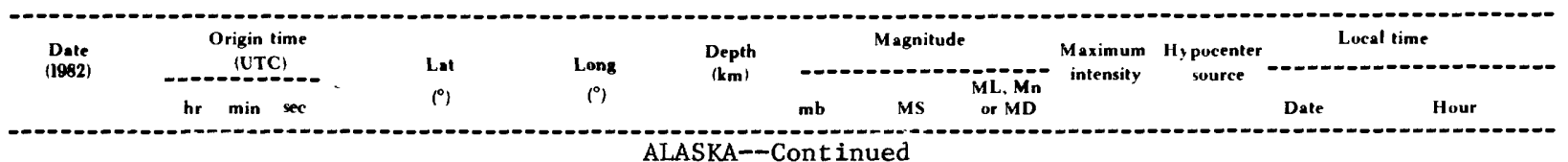

ALASKA-Continued

\begin{tabular}{|c|c|c|c|c|c|c|c|c|c|c|c|c|c|}
\hline $\begin{array}{l}\text { NOV. } \\
\text { NOV. }\end{array}$ & $\begin{array}{l}24 \\
24\end{array}$ & $\begin{array}{lll}20 & 08 & 38.4 \\
12 & 17 & 10.1 \\
22 & 15 & 10.8 \\
21 & 58 & 29.4\end{array}$ & $\begin{array}{l}65.13 \mathrm{~N} . \\
51.13 \mathrm{~N} . \\
63.98 \mathrm{~N} \\
61.86 \mathrm{~N}\end{array}$ & $\begin{array}{l}178.31 \mathrm{~W} \\
148.79 \mathrm{~W} \\
150.71 \mathrm{~W}\end{array}$ & 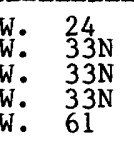 & $\begin{array}{l}\ddot{4} \ddot{2} \\
\because \cdots\end{array}$ & $\begin{array}{l}\because: \\
\because: \\
\because:\end{array}$ & ऑ. & $\begin{array}{l}\because: \\
\because: \\
\cdots\end{array}$ & & & 3 & 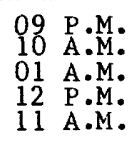 \\
\hline & $\begin{array}{r}28 \\
29 \\
29 \\
1\end{array}$ & $\begin{array}{lll}23 & 33 & 37.9 \\
18 & 41 & 29.3 \\
21 & 46 & 32.2 \\
12 & 47 & 04.2\end{array}$ & $\begin{array}{l}.58 \mathrm{~N} . \\
.88 \mathrm{~N}: \\
.75 \mathrm{~N} \\
.89 \mathrm{~N} \\
.80 \mathrm{~N}\end{array}$ & $\begin{array}{l}5.92 \mathrm{~W} \\
9.66 \mathrm{~W} \\
3.40 \mathrm{~W} \\
0.91 \mathrm{~W} \\
2.84 \mathrm{~W}\end{array}$ & 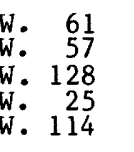 & $\begin{array}{l}4.2 \\
\because \cdots \\
\because \cdots \\
\cdots\end{array}$ & $\begin{array}{l}\cdots \\
\cdots \\
\cdots \\
\cdots\end{array}$ & $3.05 \mathrm{M}$ & $\begin{array}{l}\cdots \\
\cdots \\
\cdots\end{array}$ & & & & $\begin{array}{l}01 \\
08 \\
11 \\
02\end{array}$ \\
\hline $\begin{array}{l}\text { EC. } \\
\text { EC: } \\
\text { EC: }\end{array}$ & $\begin{array}{l}2 \\
3 \\
5 \\
6 \\
8\end{array}$ & $\begin{array}{lll}09 & 43 & 53.4 \\
23 & 52 & 32.2 \\
12 & 09 & 51.8 \\
12 & 39 & 26.4 \\
06 & 10 & 17.5\end{array}$ & $\begin{array}{l}51.88 \mathrm{~N} . \\
60.69 \mathrm{~N}: \\
60.26 \mathrm{~N} . \\
56.76 \mathrm{~N} . \\
53.85 \mathrm{~N} .\end{array}$ & $\begin{array}{l}170.45 \mathrm{~W} \\
151.45 \mathrm{~W} \\
152.15 \mathrm{~W} \\
151.99 \mathrm{~W} \\
160.45 \mathrm{~W}\end{array}$ & $\begin{array}{ll}\text { W. } & 33 \mathrm{~N} \\
\text { W. } & 97 \\
\text { W. } & 90 \\
\text { W. } & 33 \mathrm{~N} \\
\text { W. } & 33 \mathrm{~N}\end{array}$ & $\begin{array}{l}5.5 \\
4.99 \\
4.6 \\
4.8\end{array}$ & 4.8 & $\because \ddot{3}$ & $\because \ddot{\text { IV }}$ & & $\begin{array}{l}\text { DEC: } \\
\text { DEC: } \\
\text { DEC: } \\
\text { DEC: } \\
\text { DEC: }\end{array}$ & $\frac{1}{3}$ & $\begin{array}{ll}10 & \text { P.M. } \\
01 & \text { P.M. } \\
02 & \text { A.M. } \\
02 & \text { A.M. } \\
08 & \text { P.M. }\end{array}$ \\
\hline $\begin{array}{l}\text { EC: } \\
\text { ¿C: } \\
\text { QC. }\end{array}$ & $\begin{array}{l}9 \\
10 \\
11 \\
12 \\
12\end{array}$ & $\begin{array}{lll}14 & 56 & 25.6 \\
09 & 04 & 09.9 \\
20 & 35 & 38.0 \\
06 & 54 & 10.7 \\
11 & 56 & 04.3\end{array}$ & $\begin{array}{l}59.43 \mathrm{~N} . \\
62.64 \mathrm{~N} . \\
52.51 \\
59.87 \\
51.47 \\
\mathrm{~N} .\end{array}$ & $\begin{array}{l}152.74 \mathrm{~W} \\
149.31 \\
172.18 \\
153.47 \\
178.92\end{array}$ & 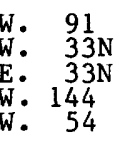 & $\begin{array}{l}\cdots . \\
4.6 \\
3.6 \\
4.6\end{array}$ & $\begin{array}{l}\ldots \\
\cdots \\
\cdots\end{array}$ & & $\begin{array}{l}\cdots \\
\cdots \\
\cdots\end{array}$ & & $\begin{array}{l}\text { DEC. } \\
\text { DEC: } \\
\text { DEC: } \\
\text { DEC: } \\
\text { DEC. }\end{array}$ & $\begin{array}{r}9 \\
9 \\
11 \\
11 \\
12\end{array}$ & $\begin{array}{ll}04 & \text { A.M. } \\
11 & \text { P.M. } \\
09 & \text { A.M. } \\
08 & \text { P.M. } \\
00 & \text { A.M. }\end{array}$ \\
\hline EC: & $\begin{array}{l}13 \\
14 \\
14 \\
15\end{array}$ & $\begin{array}{lll}15 & 30 & 07.9 \\
02 & 01 & 29.4 \\
13 & 45 & 09.0 \\
23 & 34 & 21.0 \\
12 & 13 & 26.5\end{array}$ & $\begin{array}{l}51.82 \mathrm{~N} . \\
52.02 \\
63.86 \\
61.97 \\
6 .\end{array}$ & $\begin{array}{l}175.53 \mathrm{E} \\
179.05 \mathrm{E} \\
147.31 \mathrm{~W} \\
149.15 \mathrm{~W}\end{array}$ & $\begin{array}{ll}\text { W. } & 89 \\
\text { E: } & 59 \\
\text { E. } & 33 \mathrm{~N} \\
\text { W. } & 33 \mathrm{~N} \\
\text { W. } & 16\end{array}$ & $\begin{array}{l}4 \\
4\end{array}$ & & & $\begin{array}{l}\cdots \\
\because \\
\cdots\end{array}$ & & $\begin{array}{l}\text { DEC. } \\
\text { DEC: } \\
\text { DEC: } \\
\text { DEC: } \\
\text { DEC: }\end{array}$ & $\begin{array}{l}12 \\
14 \\
14 \\
15\end{array}$ & $\begin{array}{ll}05 & \text { A.M. } \\
03 & \text { P.M. } \\
02 & \text { A.M. } \\
01 & \text { P.M. } \\
02 & \text { A.M. }\end{array}$ \\
\hline : & $\begin{array}{l}17 \\
17 \\
17\end{array}$ & $\begin{array}{lll}09 & 34 & 44.8 \\
10 & 55 & 54.7 \\
11 & 03 & 20.4 \\
12 & 17 & 19.5 \\
06 & 05 & 21.1\end{array}$ & $\begin{array}{l}62.25 \mathrm{~N} . \\
60.68 \mathrm{~N} . \\
56.45 \\
64.07 \\
61.94 \\
\mathrm{~N} .\end{array}$ & $\begin{array}{l}150.05 \mathrm{~W} \\
149.80 \mathrm{~W} \\
147.19 \mathrm{~W}\end{array}$ & $\begin{array}{l}\text { W. } 98 \\
\text { W. } 153 \\
\text { W. } 33 \mathrm{~N} \\
\text { W. } 33 \mathrm{~N} \\
\text { W. } 59\end{array}$ & $\begin{array}{l}\ddot{0} \\
\ddot{4} \ddot{4} \\
\ddot{3} \ddot{9}\end{array}$ & & & II & & & $\begin{array}{l}15 \\
17 \\
17 \\
17 \\
17\end{array}$ & $\begin{array}{ll}11 & \text { P.M. } \\
00 & \text { A.M. } \\
01 & \text { A.M. } \\
02 & \text { A.M. } \\
08 & \text { P.M. }\end{array}$ \\
\hline C. & $\begin{array}{l}18 \\
19 \\
21 \\
23 \\
24\end{array}$ & $\begin{array}{lll}21 & 57 & 25.0 \\
17 & 34 & 32.9 \\
01 & 01 & 44.7 \\
08 & 24 & 54.2 \\
23 & 31 & 03.0\end{array}$ & $\begin{array}{l}63.19 \\
57.35 \\
59.77 \\
63 . \\
63.72 \\
52.58 \\
\text { N. }\end{array}$ & $\begin{array}{l}150.84 \mathrm{~h} \\
152.06 \mathrm{~h} \\
153.52 \mathrm{~h} \\
153.11 \mathrm{~h} \\
173.29 \mathrm{~b}\end{array}$ & $\begin{array}{l}\text { W. } 142 \\
\text { W. } 33 \mathrm{~N} \\
\text { W. } 126 \\
\text { W. } 33 \mathrm{~N} \\
\text { E. } 72\end{array}$ & $\begin{array}{l}\ddot{4} \ddot{4} \\
\ddot{0} \\
\ddot{5} \ddot{2}\end{array}$ & & 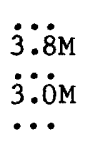 & $\cdots$ & & $\begin{array}{l}\text { C. } \\
\text { C. } \\
\text { C. } \\
\text { C. } \\
\text { C. }\end{array}$ & $\begin{array}{l}18 \\
19 \\
20 \\
22 \\
24\end{array}$ & $\begin{array}{ll}11 & \text { A.M. } \\
07 & \text { A.M. } \\
03 & \text { P.M. } \\
10 & \text { P.M. } \\
12 & \text { P.M. }\end{array}$ \\
\hline $\begin{array}{l}\text { EC. } \\
\text { EC: } \\
\text { EC: }\end{array}$ & $\begin{array}{l}25 \\
25 \\
25 \\
25 \\
25\end{array}$ & $\begin{array}{lll}02 & 17 & 31.6 \\
02 & 39 & 44.3 \\
04 & 02 & 45.9 \\
05 & 12 & 22.3 \\
09 & 59 & 16.1\end{array}$ & $\begin{array}{l}52.08 \mathrm{~N} . \\
52.98 \mathrm{~N} . \\
59.80 \mathrm{~N} . \\
61.31 \\
63.08 \mathrm{~N} .\end{array}$ & $\begin{array}{l}174.98 \\
170.88 \\
141.14 \\
147.10 \\
148.61\end{array}$ & 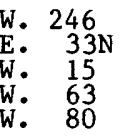 & $\begin{array}{l}4.4 \\
4.6 \\
\because \because . \\
\ddot{3} .6\end{array}$ & & & & & $\begin{array}{l}\text { DEC: } \\
\text { DEC: } \\
\text { DEC: } \\
\text { DEC: } \\
\text { DEC: }\end{array}$ & $\begin{array}{l}24 \\
24 \\
24 \\
24 \\
24\end{array}$ & $\begin{array}{ll}03 & \text { P.M. } \\
03 & \text { P.M. } \\
06 & \text { P.M. } \\
07 & \text { P.M. } \\
11 & \text { P.M. }\end{array}$ \\
\hline $\begin{array}{l}\text { EC. } \\
\text { EC: } \\
\text { EC: } \\
\text { C. }\end{array}$ & $\begin{array}{l}26 \\
26 \\
27 \\
27 \\
28\end{array}$ & $\begin{array}{lll}03 & 10 & 02.6 \\
03 & 24 & 17.7 \\
02 & 38 & 35.8 \\
18 & 24 & 19.4 \\
19 & 40 & 02.7\end{array}$ & $\begin{array}{l}55.51 \mathrm{~N} . \\
66.24 \\
61.19 \\
63.06 \\
61.58 \mathrm{~N} . \\
61 .\end{array}$ & $\begin{array}{l}159.88 \mathrm{~h} \\
150.79 \mathrm{~h} \\
152.35 \mathrm{~h} \\
149.23 \mathrm{~h} \\
146.40 \mathrm{~h}\end{array}$ & $\begin{array}{l}\text { W. } \quad 41 \\
\text { W. } \quad 33 \mathrm{~N} \\
\text { W. } 144 \\
\text { W. } 75 \\
\text { W. } 53\end{array}$ & & & & $\ldots$ & & $\begin{array}{l}\text { DEC. } \\
\text { DEC: } \\
\text { DEC: } \\
\text { DEC: } \\
\text { DEC. }\end{array}$ & $\begin{array}{l}25 \\
25 \\
26 \\
27 \\
28\end{array}$ & $\begin{array}{ll}05 & \text { P.M. } \\
05 & \text { P.M. } \\
04 & \text { P.M. } \\
08 & \text { A.M. } \\
09 & \text { A.M. }\end{array}$ \\
\hline $\begin{array}{l}\text { DEC. } \\
\text { DEC: } \\
\text { DEC: } \\
\text { DEC. }\end{array}$ & $\begin{array}{l}29 \\
30 \\
31 \\
31 \\
31\end{array}$ & $\begin{array}{lll}04 & 34 & 19.8 \\
10 & 09 & 16.3 \\
02 & 58 & 41.4 \\
07 & 22 & 17.9 \\
11 & 04 & 03.6\end{array}$ & $\begin{array}{l}64.02 \mathrm{~N} . \\
63.25 \mathrm{~N} . \\
60.01 \mathrm{~N} . \\
67.66 \mathrm{~N} . \\
67.81 \mathrm{~N} .\end{array}$ & $\begin{array}{l}149.76 \\
151.84 \\
152.07 \\
161.24 \\
161.35\end{array}$ & $\begin{array}{l}14 \\
89 \\
62 \\
33 \mathrm{~N} \\
33 \mathrm{~N}\end{array}$ & & & & & & : & $\begin{array}{l}28 \\
30 \\
30 \\
30 \\
31\end{array}$ & $\begin{array}{ll}06 & \text { P.M. } \\
00 & \text { A.M. } \\
04 & \text { P.M. } \\
08 & \text { P.M. } \\
00 & \text { A.M. }\end{array}$ \\
\hline EC. & 31 & 144420.5 & $63.13 \mathrm{~N}$. & 150.80 & W. 166 & & $\cdots$ & & •. & G & DEC. & 31 & 04 \\
\hline
\end{tabular}

ARIZONA

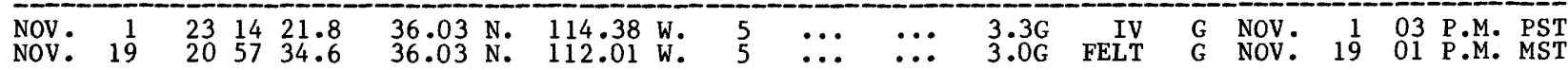

ARKANSAS

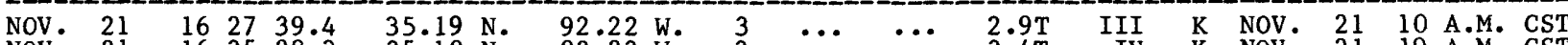

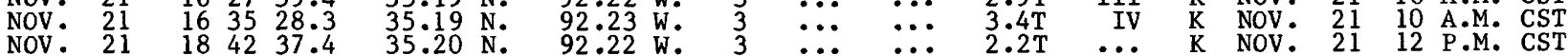


Table 1.-Summary of U.S. earthquakes for October-December 1982-Continued

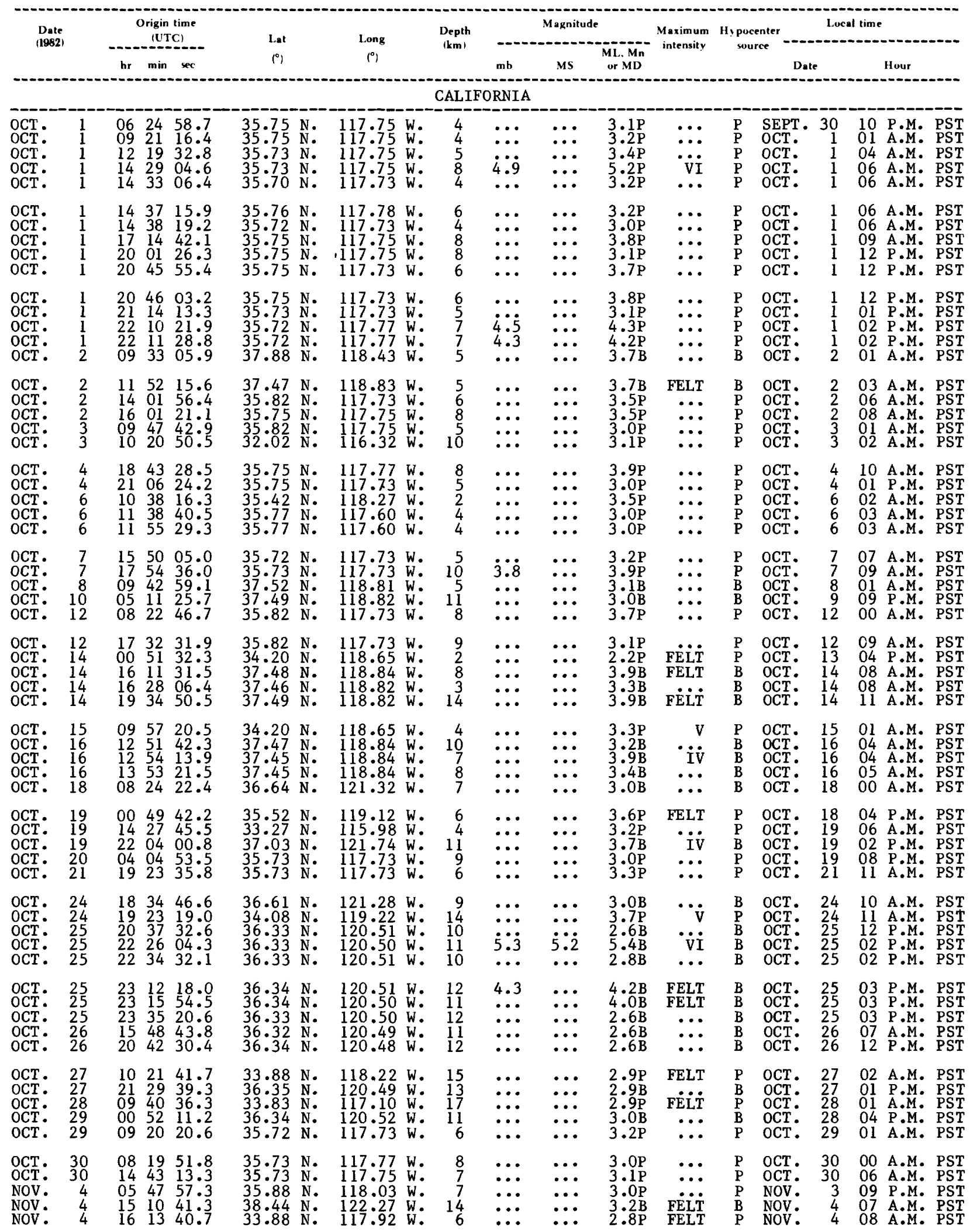


Table 1.--Summary of U. S. earthquakes for October-December 1982-Continued

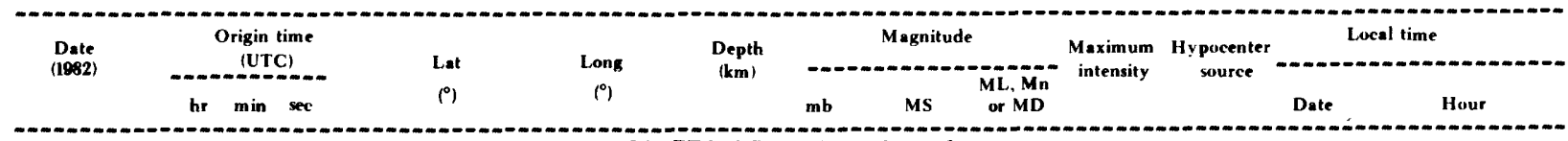

CALIFORNIA--Cont inued

\begin{tabular}{|c|c|c|c|c|c|c|c|c|c|c|c|c|c|}
\hline $\begin{array}{l}\text { NOV. } \\
\text { NOV: } \\
\text { NOV: } \\
\text { NOV: } \\
\text { NOV. }\end{array}$ & $\begin{array}{r}4 \\
9 \\
10 \\
10 \\
10\end{array}$ & $\begin{array}{lll}19 & 07 & 41.2 \\
11 & 12 & 05.8 \\
04 & 17 & 26.9 \\
11 & 21 & 25.7 \\
13 & 29 & 57.4\end{array}$ & $\begin{array}{l}35.73 \text { N. } \\
39.74 \\
38.83 \\
34 . \\
34.05 \\
37.48 \text { N. }\end{array}$ & $\begin{array}{l}117.73 \mathrm{~W} . \\
120.60 \mathrm{~W} . \\
122.77 \mathrm{~W} . \\
116.67 \mathrm{~W} . \\
118.82 \mathrm{~W} .\end{array}$ & $\begin{array}{r}9 \\
10 \\
2 \\
8 \\
5\end{array}$ & $\begin{array}{l}\cdots \\
\ddot{4}: 1 \\
\cdots\end{array}$ & $\begin{array}{l}\cdots \\
\cdots \\
\cdots\end{array}$ & $\begin{array}{l}3.0 \mathrm{P} \\
3.0 \mathrm{~B} \\
2.9 \mathrm{~B} \\
3.9 \mathrm{P} \\
3.6 \mathrm{~B}\end{array}$ & $\begin{array}{r}\ddot{\ddot{I V}} \\
\text { FELT } \\
\text { FELT }\end{array}$ & & $\begin{array}{l}\text { NOV: } \\
\text { NOV: } \\
\text { NOV: } \\
\text { NOV: } \\
\text { NOV. }\end{array}$ & $\begin{array}{r}4 \\
9 \\
9 \\
10 \\
10\end{array}$ & $\begin{array}{ll}11 & \text { A.M. PST } \\
03 & \text { A.M. PST } \\
08 & \text { P.M. PST } \\
03 & \text { A.M. PST } \\
05 & \text { A.M. PST }\end{array}$ \\
\hline $\begin{array}{l}\text { ov: } \\
\text { ov: } \\
\text { ov: } \\
\text { ov. }\end{array}$ & $\begin{array}{l}12 \\
12 \\
13 \\
15 \\
21\end{array}$ & $\begin{array}{lll}09 & 33 & 55.2 \\
21 & 57 & 11.9 \\
20 & 18 & 20.5 \\
03 & 48 & 41.7 \\
09 & 28 & 32.5\end{array}$ & $\begin{array}{l}32.98 \mathrm{~N} . \\
35.33 \mathrm{~N} . \\
36.69 \mathrm{~N} . \\
39.80 \mathrm{~N} . \\
35.82 \mathrm{~N} .\end{array}$ & $\begin{array}{l}115.57 \mathrm{~W} . \\
120.54 \mathrm{~W} . \\
121.20 \mathrm{~W} . \\
122.67 \mathrm{~W} . \\
117.73 \mathrm{~W} .\end{array}$ & $\begin{array}{r}9 \\
9 \\
16 \\
5\end{array}$ & $\begin{array}{l}\cdots \\
\cdots \\
\cdots\end{array}$ & $\begin{array}{l}\cdots \\
\cdots \\
\cdots\end{array}$ & $\begin{array}{l}2.6 \mathrm{P} \\
3.0 \mathrm{~B} \\
3.7 \mathrm{~B} \\
3.0 \mathrm{~B} \\
3.1 \mathrm{P}\end{array}$ & $\begin{array}{l}\text { FELT } \\
\text { FELT } \\
\text { III } \\
\ldots \\
\cdots\end{array}$ & & $\begin{array}{l}\text { NOV: } \\
\text { NOV: } \\
\text { NOV: } \\
\text { NOV: } \\
\text { NOV: }\end{array}$ & $\begin{array}{l}14 \\
21\end{array}$ & $\begin{array}{lll}01 & \text { A.M. } & \text { PST } \\
01 & \text { P.M. PST } \\
12 & \text { P.M. PST } & \text { PST } \\
07 & \text { P.M. PST } \\
01 & \text { A.M. PST }\end{array}$ \\
\hline $\begin{array}{l}\text { ov. } \\
\text { ov: } \\
\text { ov: } \\
\text { ov: } \\
\text { ov. }\end{array}$ & $\begin{array}{l}21 \\
23 \\
26 \\
26 \\
27\end{array}$ & $\begin{array}{lll}17 & 22 & 48.8 \\
15 & 48 & 58 \cdot 2 \\
09 & 29 & 49.1 \\
12 & 30 & 13.8 \\
17 & 52 & 24.0\end{array}$ & $\begin{array}{l}32.88 \mathrm{~N} . \\
40.72 \\
38.28 \\
34.84 \\
33.53 \\
3 .\end{array}$ & $\begin{array}{l}115.97 \mathrm{~W} . \\
124.22 \text { W. } \\
122.16 \mathrm{~W}: \\
118.96 \mathrm{~W} . \\
118.20 \mathrm{~W} .\end{array}$ & $\begin{array}{r}3 \\
8 \\
11 \\
5 \\
6\end{array}$ & $\begin{array}{l}\cdots \\
\cdots \\
\cdots\end{array}$ & $\begin{array}{l}\cdots \\
\because \cdots \\
\cdots\end{array}$ & $\begin{array}{l}3.0 \mathrm{P} \\
2.9 \mathrm{~B} \\
3.3 \mathrm{~B} \\
3.1 \mathrm{P} \\
3.1 \mathrm{P}\end{array}$ & $\because \frac{.}{\mathrm{I} \dot{\mathrm{I}}}$ & & $\begin{array}{l}\mathrm{v}: \\
\mathrm{v}: \\
\mathrm{v}: \\
\mathrm{v}:\end{array}$ & $\begin{array}{l}21 \\
23 \\
26 \\
26 \\
27\end{array}$ & $\begin{array}{ll}09 & \text { A.M. PST } \\
07 & \text { A.M. PST } \\
01 & \text { A.M. PST } \\
04 & \text { A.M. PST } \\
09 & \text { A.M. PST }\end{array}$ \\
\hline $\begin{array}{l}\text { OV. } \\
\text { EC: } \\
\text { EC. } \\
\text { EC. }\end{array}$ & $\begin{array}{r}28 \\
2 \\
2 \\
7 \\
9\end{array}$ & $\begin{array}{lll}14 & 43 & 19.1 \\
16 & 02 & 45.9 \\
22 & 44 & 25.2 \\
23 & 05 & 39.1 \\
10 & 15 & 10.5\end{array}$ & $\begin{array}{ll}44 & \mathrm{~N} . \\
17 & \mathrm{~N} \\
27 & \mathrm{~N} \\
92 & \mathrm{~N} \\
02 & \mathrm{~N} .\end{array}$ & $\begin{array}{l}118.85 \mathrm{~W} . \\
122.21 \mathrm{~W} . \\
119.57 \mathrm{~W} . \\
121.70 \mathrm{~W}: \\
117.13 \mathrm{~W} .\end{array}$ & $\begin{array}{r}3 \\
10 \\
6 \\
10 \\
20\end{array}$ & $\begin{array}{l}\ldots \\
\cdots \\
\cdots\end{array}$ & $\begin{array}{l}\ldots \\
\cdots \\
\cdots\end{array}$ & $\begin{array}{l}3.5 \mathrm{~B} \\
3.0 \mathrm{~B} \\
3.4 \mathrm{P} \\
3.1 \mathrm{~B} \\
3.1 \mathrm{P}\end{array}$ & $\begin{array}{l}\text { FELT } \\
\text { FELT } \\
\text { FĖL̇T }\end{array}$ & & $\begin{array}{l}\text { OV. } \\
\text { EC: } \\
\text { EC: } \\
\text { EC: }\end{array}$ & $\begin{array}{l}2 \\
2 \\
2 \\
9 \\
9\end{array}$ & $\begin{array}{lll}06 & \text { A.M. PST } \\
08 & \text { A.M. PST } \\
02 & \text { P.M. PST } \\
03 & \text { P.M. PST } \\
02 & \text { A.M. PST }\end{array}$ \\
\hline $\begin{array}{l}E C . \\
E C . \\
E C . \\
E C .\end{array}$ & $\begin{array}{l}12 \\
12 \\
14 \\
14 \\
16\end{array}$ & $\begin{array}{lll}10 & 38 & 06.0 \\
20 & 59 & 08.8 \\
06 & 46 & 09.9 \\
19 & 15 & 31.8 \\
06 & 53 & 01.3\end{array}$ & $\begin{array}{l}37.54 \mathrm{~N} . \\
37.53 \mathrm{~N} . \\
36.91 \mathrm{~N} . \\
40.54 \mathrm{~N} . \\
40.50 \mathrm{~N} .\end{array}$ & $\begin{array}{l}118.82 \text { W. } \\
118.81 \text { W. } \\
121.49 \text { W. } \\
124.20 \text { W. } \\
124.26 \text { W. }\end{array}$ & $\begin{array}{r}6 \\
4 \\
5 \\
20 \\
18\end{array}$ & $\begin{array}{l}\ldots \\
\because \cdots \\
\ddot{4} . \dot{8}\end{array}$ & $\begin{array}{l}\cdots \\
\cdots \\
\ddot{4} . \dot{5}\end{array}$ & $\begin{array}{l}3.6 \mathrm{~B} \\
3.7 \mathrm{~B} \\
3.2 \mathrm{~B} \\
3.6 \mathrm{~B} \\
4.4 \mathrm{~B}\end{array}$ & $\begin{array}{l}\text { FELT } \\
\text { FELT } \\
\text { FELT } \\
\text { FELT } \\
\text { VI }\end{array}$ & & & $\begin{array}{l}12 \\
12 \\
13 \\
14 \\
15\end{array}$ & $\begin{array}{lll}02 & \text { A.M. PST } & \text { PST } \\
12 & \text { P.M. PST } \\
10 & \text { P.M. PST } \\
11 & \text { A.M. PST } \\
10 & \text { P.M. PST }\end{array}$ \\
\hline $\begin{array}{l}\text { EC. } \\
\text { EC: } \\
\text { EC: } \\
\text { EC. }\end{array}$ & $\begin{array}{l}19 \\
19 \\
20 \\
20 \\
21\end{array}$ & $\begin{array}{lll}02 & 28 & 23.0 \\
09 & 38 & 50.3 \\
00 & 08 & 21: 1 \\
04 & 11 & 01.3 \\
21 & 07 & 14.7\end{array}$ & $\begin{array}{l}35.08 \mathrm{~N} . \\
35.80 \mathrm{~N} . \\
40.54 \\
37.57 \\
37.53 \mathrm{~N} .\end{array}$ & $\begin{array}{l}116.82 \mathrm{~W} . \\
120.43 \mathrm{~W} . \\
123.97 \mathrm{~W} \\
118.87 \mathrm{~W}: \\
118.87 \mathrm{~W} .\end{array}$ & $\begin{array}{l}3 \\
6 \\
6\end{array}$ & $\because:$ & & $\begin{array}{l}3.1 \mathrm{P} \\
3.0 \mathrm{P} \\
3.3 \mathrm{~B} \\
3.1 \mathrm{P} \\
3.0 \mathrm{P}\end{array}$ & FE்L̇ & & $\begin{array}{l}\text { DEC: } \\
\text { DEC: } \\
\text { DEC: } \\
\text { DEC: } \\
\text { DEC. }\end{array}$ & $\begin{array}{l}18 \\
19 \\
19 \\
19 \\
21\end{array}$ & $\begin{array}{lll}06 & \text { P.M. PST } \\
01 & \text { A.M. PST } \\
04 & \text { P.M. PST } \\
08 & \text { P.M. PST } \\
01 & \text { P.M. PST }\end{array}$ \\
\hline $\begin{array}{l}E C . \\
E C . \\
E C . \\
E C .\end{array}$ & $\begin{array}{l}21 \\
22 \\
22 \\
22 \\
23\end{array}$ & $\begin{array}{lll}22 & 28 & 12.9 \\
09 & 40 & 49.9 \\
12 & 46 & 59.9 \\
14 & 47 & 36.9 \\
10 & 47 & 03.1\end{array}$ & $\begin{array}{l}37.64 \mathrm{~N} . \\
37.36 \mathrm{~N} . \\
33.47 \mathrm{~N} . \\
35.75 \mathrm{~N} . \\
37.48 \mathrm{~N} .\end{array}$ & $\begin{array}{l}118.96 \mathrm{~W} . \\
118.52 \mathrm{~W} \\
116.57 \mathrm{~W} \\
117.75 \mathrm{~W} . \\
118.87 \mathrm{~W} .\end{array}$ & $\begin{array}{r}5 \\
10 \\
12 \\
10 \\
8\end{array}$ & $\because \cdots$ & ... & $\begin{array}{l}3.3 \mathrm{~B} \\
3.4 \mathrm{~B} \\
3.0 \mathrm{P} \\
3.3 \mathrm{P} \\
3.2 \mathrm{P}\end{array}$ & $\begin{array}{l}\text { FELT } \\
\text { FELT } \\
\text { FËL் }\end{array}$ & & & $\begin{array}{l}21 \\
22 \\
22 \\
22 \\
23\end{array}$ & 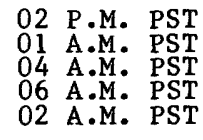 \\
\hline $\begin{array}{l}\text { DEC. } \\
\text { DEC: } \\
\text { DEC: } \\
\text { DEC: } \\
\text { DEC. }\end{array}$ & $\begin{array}{l}23 \\
26 \\
26 \\
26 \\
26\end{array}$ & $\begin{array}{lll}13 & 52 & 01.0 \\
01 & 54 & 55.3 \\
09 & 43 & 04.6 \\
09 & 59 & 46.6 \\
10 & 04 & 24.9\end{array}$ & $\begin{array}{l}35.88 \mathrm{~N} . \\
35.82 \\
35.70 \\
38.81 \\
36.81 \\
3 .\end{array}$ & $\begin{array}{l}120.38 \mathrm{~W} . \\
117.73 \mathrm{~W} . \\
117.73 \mathrm{~W} . \\
122.78 \mathrm{~W} . \\
121.55 \mathrm{~W} .\end{array}$ & $\begin{array}{l}5 \\
1 \\
6\end{array}$ & $\because$ & ... & $\begin{array}{l}2.8 \mathrm{P} \\
3.1 \mathrm{~B} \\
3.5 \mathrm{~B}\end{array}$ & $\begin{array}{r}\ldots \\
\because \because \dot{~ I V ~} \\
\text { FELT }\end{array}$ & $P$ & $\begin{array}{l}\text { DEC. } \\
\text { DEC: } \\
\text { DEC: } \\
\text { DEC: } \\
\text { DEC. }\end{array}$ & $\begin{array}{l}23 \\
25 \\
26 \\
26 \\
26\end{array}$ & $\begin{array}{lll}05 & \text { A.M. } & \text { PST } \\
05 & \text { P.M. PST } \\
01 & \text { A.M. PST } \\
01 & \text { A.M. PST } \\
02 & \text { A.M. PST }\end{array}$ \\
\hline $\begin{array}{l}\text { DEC. } \\
\text { DEC. } \\
\text { DEC: } \\
\text { DEC. } \\
\text { DEC. }\end{array}$ & $\begin{array}{l}28 \\
28 \\
28 \\
28 \\
28\end{array}$ & $\begin{array}{lll}00 & 49 & 38.8 \\
07 & 15 & 14.8 \\
07 & 20 & 23.6 \\
16 & 08 & 59.8 \\
20 & 39 & 15.3\end{array}$ & $\begin{array}{l}37.49 \mathrm{~N} . \\
37.49 \\
35.82 \\
35.82 \\
35.82 \\
35\end{array}$ & $\begin{array}{l}118.80 \mathrm{~W} . \\
118.81 \mathrm{~W} . \\
117.75 \mathrm{~W} . \\
117.75 \mathrm{~W} . \\
117.75 \mathrm{~W} .\end{array}$ & 3 & ... & & $\begin{array}{l}3.6 \mathrm{~B} \\
3.3 \mathrm{~B} \\
\because \because\end{array}$ & & & $\begin{array}{l}\text { DEC. } \\
\text { DEC: } \\
\text { DEC: } \\
\text { DEC: } \\
\text { DEC. }\end{array}$ & $\begin{array}{l}27 \\
27 \\
27 \\
28 \\
28\end{array}$ & $\begin{array}{lll}04 & \text { P.M. } & \text { PST } \\
11 & \text { P.M. } & \text { PST } \\
11 & \text { P.M. PST } \\
08 & \text { A.M. PST } \\
12 & \text { P.M. PST }\end{array}$ \\
\hline $\begin{array}{l}E C . \\
E C . \\
E C . \\
E C .\end{array}$ & $\begin{array}{l}30 \\
31 \\
31 \\
31 \\
31\end{array}$ & $\begin{array}{lll}04 & 00 & 29.5 \\
06 & 21 & 49.6 \\
09 & 07 & 23.3 \\
19 & 50 & 08.8 \\
19 & 52 & 56.7\end{array}$ & $\begin{array}{l}33.95 \mathrm{~N} . \\
35.82 \\
35.82 \\
35.82 \\
35.80 \\
35.8\end{array}$ & $\begin{array}{l}117.73 \\
117.75\end{array}$ & $\begin{array}{l}0 \\
6 \\
6\end{array}$ & $\begin{array}{l}4.0 \\
\ddot{4} .4\end{array}$ & ... & 4. & $\begin{array}{l}\text { III } \\
\text { IV }\end{array}$ & & $\begin{array}{l}\text { DEC. } \\
\text { DEC: } \\
\text { DEC: } \\
\text { DEC: } \\
\text { DEC: }\end{array}$ & $\begin{array}{l}29 \\
30 \\
31 \\
31\end{array}$ & $\begin{array}{lll}08 & \text { P.M. } & \text { PST } \\
10 & \text { P.M. } & \text { PST } \\
01 & \text { A.M. } & \text { PST } \\
11 & \text { A.M. PST } \\
11 & \text { A.M. PST }\end{array}$ \\
\hline C: & 3 & $\begin{array}{lll}19 & 56 & 07.6 \\
20 & 46 & 52.8\end{array}$ & $\begin{array}{l}35.80 \\
35.82\end{array}$ & N. & 6 & & & & & & $\begin{array}{l}\mathrm{DEC} \text {. } \\
\mathrm{DEC} \text {. }\end{array}$ & 31 & $\begin{array}{ll}11 & \text { A.M. PST } \\
12 & \text { P.M. PST }\end{array}$ \\
\hline
\end{tabular}

CALIFORNIA--OFF THE COAST

\begin{tabular}{|c|c|c|c|c|c|c|c|c|c|c|c|}
\hline $\begin{array}{ll}\mathrm{CT}: & 11 \\
\mathrm{CT}: & 12 \\
\mathrm{C}: & 17 \\
\mathrm{C}: & 27\end{array}$ & $\begin{array}{lll}11 & 22 & 57.9 \\
14 & 07 & 40.9 \\
12 & 18 & 29.8 \\
20 & 22 & 10.5 \\
10 & 05 & 49.2\end{array}$ & $\begin{array}{l}41.56 \mathrm{~N} . \\
40.39 \mathrm{~N} . \\
40.56 \mathrm{~N} . \\
41.84 \mathrm{~N} . \\
40.40 \mathrm{~N} .\end{array}$ & 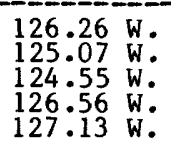 & $\begin{array}{r}5 \\
15 \\
25\end{array}$ & $\ddot{3}$ & $\begin{array}{l}3.7 \\
\ldots .\end{array}$ & $\begin{array}{l}3 . \\
3 . \\
0\end{array}$ & 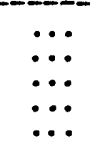 & & 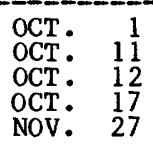 & $\begin{array}{l}\text { M. PST } \\
\text { M. PST } \\
\text { M. PST } \\
\text { M. PST } \\
\text { PST }\end{array}$ \\
\hline $\begin{array}{lr}\text { C. } & 1 \\
\text { C. } & 4 \\
\text { C. } & 10 \\
\text { C. } & 24\end{array}$ & $\begin{array}{lll}10 & 37 & 41.9 \\
03 & 08 & 23.2 \\
21 & 27 & 56.8 \\
06 & 02 & 32.3\end{array}$ & $\begin{array}{l}40.30 \mathrm{~N} \\
40.47 \mathrm{~N} \\
41.86 \mathrm{~N}\end{array}$ & $\begin{array}{l}124.52 \mathrm{~W} . \\
125.34 \mathrm{~W} \\
127.08 \mathrm{~W}\end{array}$ & & & & $\ddot{3}$ & LT & & $\begin{array}{lr}\text { DEC. } & 1 \\
\text { DEC. } & 3 \\
\text { DEC: } & 10 \\
\text { DEC: } & 23\end{array}$ & $\begin{array}{ll}07 & \mathrm{P}: \mathrm{N} \\
01 & \mathrm{P} \\
10 & \mathrm{P}: \mathrm{N}\end{array}$ \\
\hline
\end{tabular}


Table 1.--Summary of U. S. earthquakes for October-December 1982--Continued

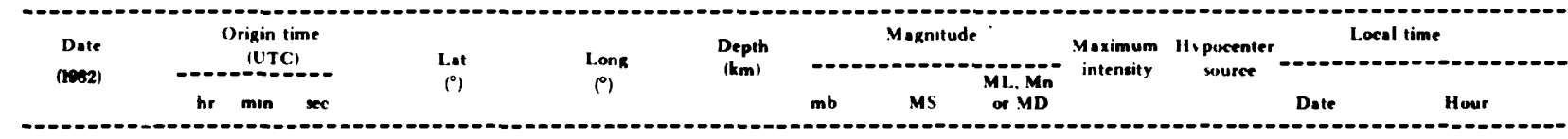

COLORADO

NOV. $22 \quad 100901.4 \quad 39.74$ N. 107.58 W. 5 ...

GEORGIA

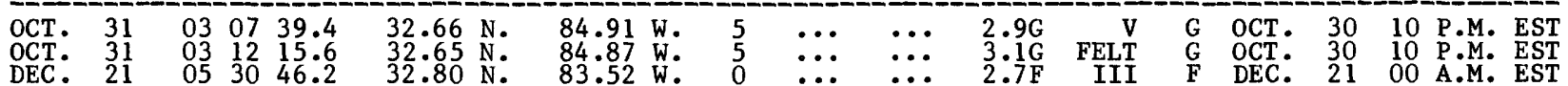

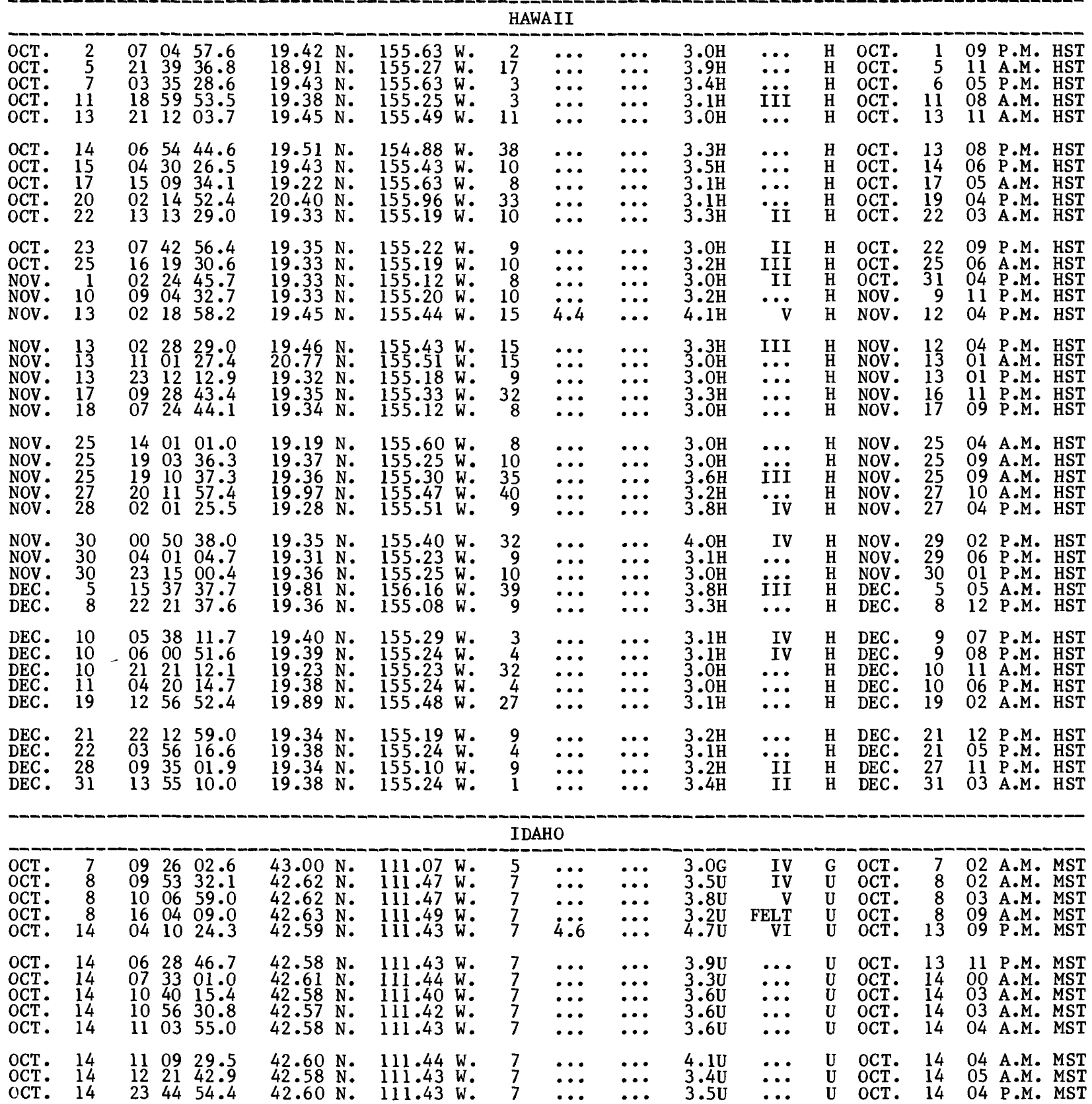


Table 1.--Summary of U. S. earthquakes for October-December 1982--Continued

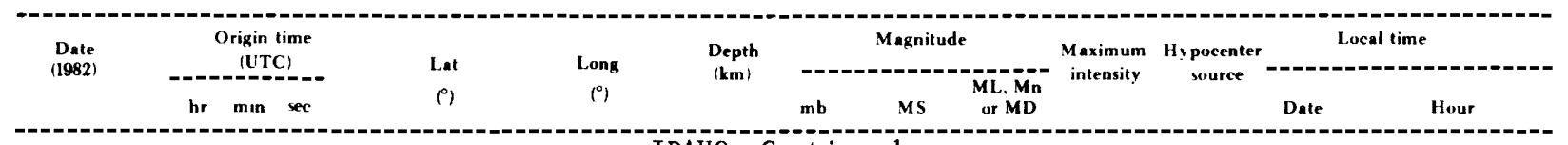

IDAHO--Cont inued

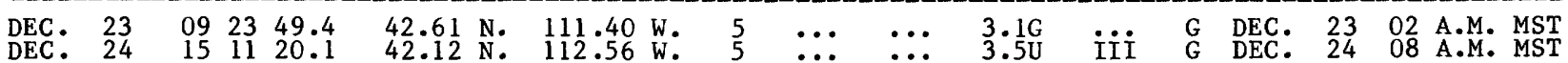

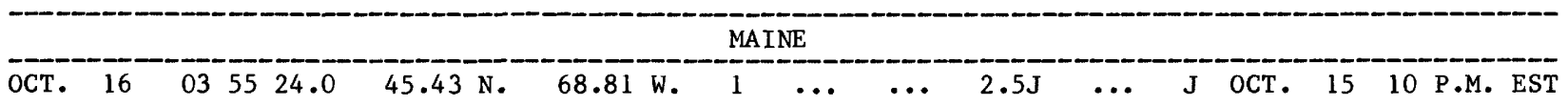
MASSACHUSETTS

\begin{tabular}{|c|c|c|c|c|c|c|c|c|c|c|c|c|c|c|c|c|c|}
\hline $\begin{array}{l}\text { OCT. } \\
\text { OCT: } \\
\text { NOV: } \\
\text { NOV: } \\
\text { NOV. }\end{array}$ & $\begin{array}{r}27 \\
28 \\
1 \\
1 \\
1\end{array}$ & $\begin{array}{l}20 \\
02 \\
03 \\
04 \\
06\end{array}$ & $\begin{array}{l}27 \\
20 \\
59 \\
38 \\
25\end{array}$ & $\begin{array}{l}24.5 \\
07.9 \\
21.9 \\
08.1 \\
55.2\end{array}$ & $\begin{array}{l}42.71 \\
42.76 \\
42.71 \\
42.76 \\
42.05\end{array}$ & $\begin{array}{l}\text { N. } \\
\text { N. } \\
\text { N: } \\
\text { N. }\end{array}$ & $\begin{array}{l}70.06 \\
70.15 \\
70.11 \\
70.17 \\
70.54\end{array}$ & $\begin{array}{l}\text { W. } \\
\text { W: } \\
\text { W: } \\
\text { W. } \\
\text { W. }\end{array}$ & $\begin{array}{r}0 \\
12 \\
4 \\
0 \\
7\end{array}$ & $\begin{array}{l}\ldots \\
\cdots \\
\cdots \\
\cdots\end{array}$ & $\begin{array}{l}\ldots \\
\cdots \\
\cdots \\
\cdots\end{array}$ & $\begin{array}{l}2.8 \mathrm{~J} \\
2.2 \mathrm{~J} \\
2.6 \mathrm{~J} \\
2.2 \mathrm{~J} \\
2.3 \mathrm{~J}\end{array}$ & $\begin{array}{l}\cdots \\
\cdots \\
\cdots\end{array}$ & $\begin{array}{l}\mathrm{J} \\
\mathrm{J} \\
\mathrm{J} \\
\mathrm{J} \\
\mathrm{J}\end{array}$ & $\begin{array}{l}\text { OCT. } \\
\text { OCT: } \\
\text { NOV: } \\
\text { NOV: } \\
\text { NOV. }\end{array}$ & $\begin{array}{r}27 \\
27 \\
0 \\
0 \\
1\end{array}$ & $\begin{array}{lll}03 & \text { P.M. EST } \\
09 & \text { P.M. EST } & \text { EST } \\
10 & \text { P.M. EST } \\
11 & \text { P.M. EST } \\
01 & \text { A.M. EST }\end{array}$ \\
\hline Nov. & 9 & 03 & 42 & 18.9 & 42.53 & N. & 72.19 & W. & 0 & $\ldots$ & . & $2.3 \mathrm{~J}$ & $\ldots$ & $\mathrm{J}$ & NOV. & 8 & 10 P.M. EST \\
\hline
\end{tabular}
MONTANA

\begin{tabular}{|c|c|c|c|c|c|c|c|c|c|c|c|c|c|c|c|c|c|}
\hline $\begin{array}{l}\text { CT: } \\
\text { CT: } \\
\text { OV. }\end{array}$ & $\begin{array}{l}21 \\
23 \\
26 \\
4 \\
26\end{array}$ & $\begin{array}{l}12 \\
08 \\
09 \\
20\end{array}$ & $\begin{array}{l}05 \\
28 \\
26 \\
58 \\
02\end{array}$ & $\begin{array}{l}28.2 \\
43.5 \\
29.9 \\
29.9 \\
04.3\end{array}$ & $\begin{array}{l}44.72 \\
47.15 \\
44.75 \\
44.72 \\
46.27\end{array}$ & $\begin{array}{l}\text { N. } \\
\text { N. } \\
\text { N. } \\
\text { N: } \\
\text { N. }\end{array}$ & $\begin{array}{l}112.71 \\
111.75 \\
111.72 \\
111.99\end{array}$ & $\begin{array}{l}\text { W. } \\
\text { W. } \\
\text { W. } \\
\text { W. } \\
\text { W. }\end{array}$ & $\begin{array}{l}5 \\
5 \\
5 \\
5 \\
5\end{array}$ & 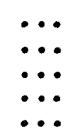 & $\begin{array}{l}\ldots \\
\ldots \\
\cdots\end{array}$ & $\begin{array}{l}4 . \\
3 . \\
4 . \\
4 . \\
3 .\end{array}$ & $\begin{array}{l}\text { IV } \\
\ddot{I V} \\
\text { IV }\end{array}$ & $\begin{array}{l}G \\
G \\
G \\
G \\
G\end{array}$ & $\begin{array}{l}\text { OCT. } \\
\text { OCT: } \\
\text { OCT: } \\
\text { NOV. } \\
\text { NOV. }\end{array}$ & $\begin{array}{r}23 \\
26 \\
4 \\
26\end{array}$ & $\begin{array}{ll}11 & \mathrm{P} \\
05 & \mathrm{~A} \\
01 & \mathrm{~A} \\
02 & \mathrm{~A} \\
01 & \mathrm{P}\end{array}$ \\
\hline
\end{tabular}

\begin{tabular}{|c|c|c|c|c|c|c|c|c|c|c|c|c|c|}
\hline \multicolumn{14}{|c|}{ NEVADA } \\
\hline $\begin{array}{l}\text { DEC. } \\
\text { DEC. } \\
\text { DEC. } \\
\text { DEC. }\end{array}$ & $\begin{array}{l}10 \\
19 \\
19 \\
28\end{array}$ & $\begin{array}{lll}15 & 20 & 00.0 \\
04 & 31 & 15.3 \\
17 & 38 & 48 \cdot 3 \\
19 & 06 & 24.8\end{array}$ & $\begin{array}{l}37.03 \mathrm{~N} . \\
37.13 \mathrm{~N} . \\
36.85 \mathrm{~N} . \\
38.03 \mathrm{~N} .\end{array}$ & $\begin{array}{l}116.07 \\
116.00 \\
115.32 \\
118.42\end{array}$ & $\begin{array}{l}\text { W. } \\
\text { W. } \\
\text { W. } \\
\text { W. }\end{array}$ & $\begin{array}{l}0 \\
6 \\
6 \\
8\end{array}$ & $\begin{array}{l}4.6 \\
\cdots .6 \\
4.7\end{array}$ & $\ldots$ & $\begin{array}{l}4.7 \mathrm{~B} \\
3.2 \mathrm{P} \\
3.0 \mathrm{P} \\
4.9 \mathrm{~B}\end{array}$ & $\ddot{\cdots}$ & $\begin{array}{l}\mathbf{E} \\
\mathrm{P} \\
\mathrm{P} \\
\mathrm{B}\end{array}$ & $\begin{array}{ll}\text { DEC. } & 10 \\
\text { DEC. } & 18 \\
\text { DEC. } & 19 \\
\text { DEC. } & 28\end{array}$ & $\begin{array}{l}07 \text { A.M. } \\
08 \text { P.M. } \\
09 \text { A.M. } \\
11 \text { A.M. }\end{array}$ \\
\hline
\end{tabular}

\section{NEW HAMPSHIRE}

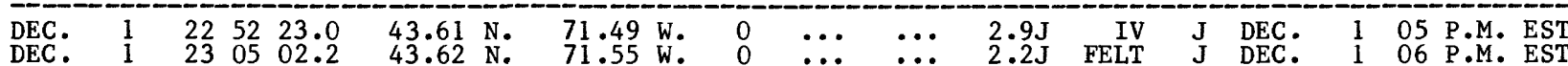

$$
\text { NEW MEXICO }
$$

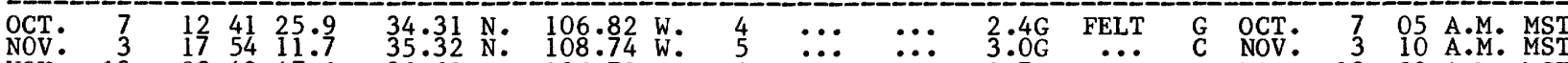

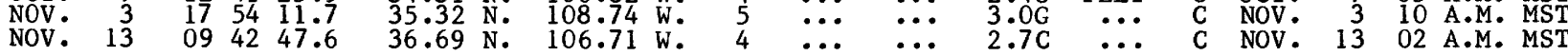

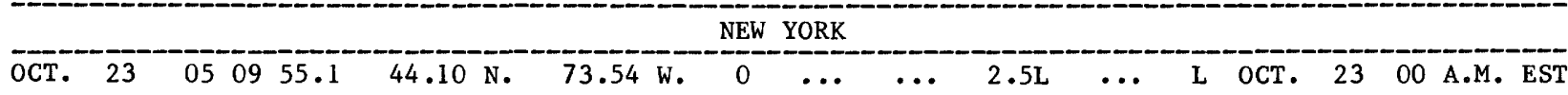

OREGON

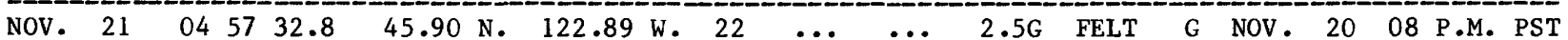

OREGON-OFF THE COAST

\begin{tabular}{|c|c|c|c|c|c|c|c|c|c|c|c|c|c|c|c|}
\hline $\begin{array}{l}\text { OCT. } \\
\text { NOV. } \\
\text { NOV: } \\
\text { NOV. } \\
\text { NOV. }\end{array}$ & $\begin{array}{l}17 \\
13 \\
13 \\
14 \\
14\end{array}$ & $\begin{array}{ll}05 & 00 \\
04 & 24 \\
15 & 44 \\
00 & 00 \\
03 & 50\end{array}$ & $\begin{array}{l}32.3 \\
18.8 \\
45.9 \\
31.8 \\
36.9\end{array}$ & $\begin{array}{l}44.65 \mathrm{~N} . \\
44.12 \mathrm{~N} . \\
44.41 \mathrm{~N} . \\
44.42 \mathrm{~N} . \\
43.67 \mathrm{~N} .\end{array}$ & $\begin{array}{l}130.07 \\
128.99 \\
129.51 \\
129.35 \\
127.69\end{array}$ & $\begin{array}{l}\text { W. } \\
\text { W. } \\
\text { W. } \\
\text { W. }\end{array}$ & $\begin{array}{l}10 \\
10 \\
10 \\
10 \\
10\end{array}$ & $\begin{array}{l}4 \cdot 8 \\
4.2 \\
5.2 \\
4.8 \\
4.3\end{array}$ & $\begin{array}{l}4.8 \\
\dot{5} . \dot{2} \\
4.6 \\
3.5\end{array}$ & $\begin{array}{l}\cdots \\
\cdots \\
\cdots \\
\cdots\end{array}$ & $\begin{array}{l}\cdots \\
\cdots \\
\cdots\end{array}$ & $\begin{array}{l}\mathrm{G} \\
\mathrm{G} \\
\mathrm{G} \\
\mathrm{G}\end{array}$ & $\begin{array}{l}\text { OCT. } \\
\text { NOV: } \\
\text { NOV: } \\
\text { NOV. } \\
\text { NOV. }\end{array}$ & $\begin{array}{l}16 \\
12 \\
13 \\
13 \\
13\end{array}$ & $\begin{array}{lll}09 & \text { P.M. } & \text { PST } \\
08 & \text { P.M. } & \text { PST } \\
07 & \text { A.M. } & \text { PST } \\
04 & \text { P.M. } & \text { PST } \\
07 & \text { P.M. } & \text { PST }\end{array}$ \\
\hline
\end{tabular}


Table 1.-Summary of U. S. earthquakes for October-December 1982--Continued

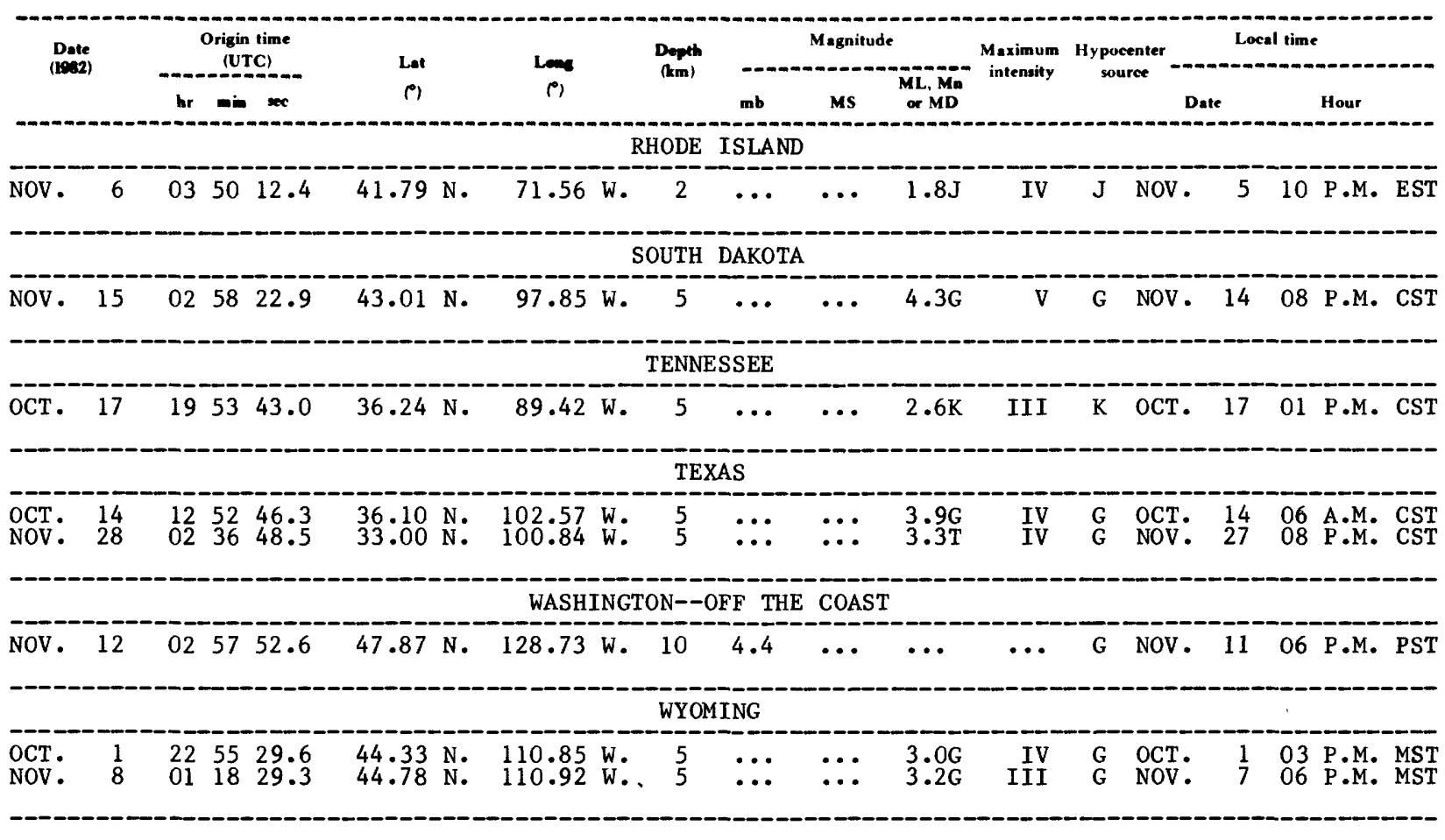


Table 2.--Summary of macroseismic data for U. S. earthquakes. October-December 1982

(Sources of the hypocenters, magnitudes, and macroseismic data: (B) University of California, Berkeley; (D) University of Montana, Missoula. (F) Georgia Institute of Technology, Atlanta; (G) U.S. Geological Survey, Golden, Colo. and Menio Park, Calif.; (H) U.S. Geological Survey, Hawaiian Volcano Observatory: (J) Weston Observatory, Mass.; (K) Tennessee Earthquake Intormation Center. Memphis; (M) National Oceanic and Atmospheric Administration, Alaska Tsunami Warning Center, Palmer; ( $P$ ) Atmospheric Administration, Alaska Tsunami Warning Center, Palmer; (P) California Institute of Technology, Pasadena; (T) Oklahoma Geological Survey, Leonard; (U) University of Utah, Salt Lake City. Normal depth = $33 \mathrm{~km}$. Dates and origin times are listed in Universal coordinated Time (UTC) giving the hour, minute, and second. Epicenters are shown in decimal degrees. Only earthquakes with intensity data and explosions are listed]

\section{ALABAMA}

31 October (G) Western Georgia Origin time: 030739.4

See Georgia listing.

31 October (G) Western Georgia Origin time: 031215.6

See Georgia listing.

\section{ALASKA}

4 October (G) Andreanof Islands, Aleutian Islands Origin time: 074652.8

Epicenter: $51.44 \mathrm{~N} ., 176.62 \mathrm{~W}$. Depth: $38 \mathrm{~km}$

Magnitude :

$$
5.5 \mathrm{mb}(\mathrm{G}), 5.0 \mathrm{MS}(\mathrm{G}), 4.9 \mathrm{MS}(\mathrm{B})
$$

$$
5.2 \mathrm{ML}(\mathrm{M})
$$

Felt on Adak Island (M).

4 November (G) Southern Alaska

Origin time: 000456.5

Epicenter: $61.53 \mathrm{~N} ., 151.62 \mathrm{~W}$.

Depth:

$120 \mathrm{~km}$

Magnitude: None computed

Felt at Anchorage and Palmer (M).

5 November (G) Central Alaska

origin time: 130646.7

Epicenter: $\quad 67.81$ N., 150.06 W.

Depth: Normal

Magnitude: $\quad 4.5 \mathrm{ML}(\mathrm{M})$

Felt at Wiseman.

10 November (G) Southern Alaska

Origin time: 172352.5

Epicenter: $\quad 60.90 \mathrm{~N} ., 146.42 \mathrm{~W}$.

Depth: $\quad 40 \mathrm{~km}$

Magnitude: $\quad 4.8 \mathrm{mb}(\mathrm{G}), 4.3 \mathrm{ML}(\mathrm{M})$

Felt at Cordova and Valdez (M).

5 December (G) Southern Alaska

Origin time: 120951.8

Epicenter: $\quad 60.26 \mathrm{~N} ., 152.15 \mathrm{~W}$.
Table 2.--Summary of macroseismic data for U. S. earthquakes, October-December 1982--Continued

\section{ALASKA--Continued}

$\begin{array}{ll}\text { Depth: } & 90 \mathrm{~km} \\ \text { Magnitude: } & 4.9 \mathrm{mb}(\mathrm{G})\end{array}$

Intensity IV: Homer (press report).

16 December (G) Southern Alaska

Origin time: 093444.8

Epicenter: $\quad 62.25 \mathrm{~N} ., 150.97 \mathrm{~W}$.

Depth: $\quad 98 \mathrm{~km}$

Magnitude: None computed

Intensity II: Palmer and Sutton (M).

24 December (G) Near Islands, Aleutian Islands

Origin time: 233103.0

Epicenter: $\quad 52.58 \mathrm{~N} ., 173.29 \mathrm{E}$.

Depth: $\quad 72 \mathrm{~km}$

Magnitude: $\quad 5.2 \mathrm{mb}(\mathrm{G})$

Intensity IV: Shemya AFB, Shemya Island.

28 December (G) Southern Alaska

Origin time: 194002.7

Epicenter: $\quad 61.58 \mathrm{~N} ., 146.40 \mathrm{~W}$.

Depth: $\quad 53 \mathrm{~km}$

Magnitude: $\quad 3.9 \mathrm{mb}(\mathrm{G}), 4.4 \mathrm{ML}(\mathrm{M})$

Intensity III: $\operatorname{Valdez}(M)$.

\section{ARIZONA}

1 November (G) Northwestern Arizona

Origin time: 231421.8

Epicenter: $\quad 36.03 \mathrm{~N} ., 114.38 \mathrm{~W}$.

Depth: $\quad 5 \mathrm{~km}$

Magnitude: $\quad 3.3 \mathrm{ML}(\mathrm{G})$

Intensity IV: Temple Bar.

19 November (G) Northwestern Arizona

Origin time: 205734.6

Epicenter: $\quad 36.03 \mathrm{~N} ., 112.01 \mathrm{~W}$.

Depth: $\quad 5 \mathrm{~km}$

Magnitude: $\quad 3.0 \mathrm{ML}(\mathrm{G})$

Felt at the Grand Canyon (University of Northern Arizona--telephone report).

\section{ARKANSAS}

21 November (K) Central Arkansas

Origin time: 162739.4

Epicenter: $\quad 35.19 \mathrm{~N}$., $92.22 \mathrm{~W}$.

Depth: $\quad 3 \mathrm{~km}$

Magnitude: $\quad 2.9 \mathrm{Mn}(\mathrm{T})$

Intensity III: Naylor (press report). Felt: Enola (press report). 
Table 2. --Summary of macroseismic data for U. S. earthquakes, Octoher-December 1982--Continued

ARKANSAS--Continued

21 November (K) Central Arkansas

Origin time: 163528.3

Epicenter: $\quad 35.19 \mathrm{~N} ., 92.23 \mathrm{~W}$.

Depth: $\quad 3 \mathrm{~km}$

Magnitude: $\quad 3.4 \mathrm{Mn}(\mathrm{T})$

Intensity IV: Naylor (press report).

Intensity III: Mount Vernon.

Felt: Enola (press report).

\section{CALIFORNIA}

1 October (P) Southern California

Origin time: 142904.6

Epicenter: $\quad 35.73 \mathrm{~N} ., 117.75 \mathrm{~W}$.

Depth:

$8 \mathrm{~km}$

Magnitude: $\quad 4.9 \mathrm{mb}(G), 5.4 \mathrm{ML}(\mathrm{B}), 5.2 \mathrm{ML}(\mathrm{P})$

This earthquake was felt over an area of about $29,800 \mathrm{~km}^{2}$ in Inyo, Kern, Los Angeles, San Bernardino, and Tulare Counties (fig. 7).

The quake, the largest of five tremors in four days, was described as a "sharp jo1t" by a Ridgecrest resident (press report).

At the China Lake Naval Weapons Center, a wall in a newly constructed building was cracked. There was no damage to the underground storage facilities where missiles and bombs are stored (press report).

\section{Intensity VI:}

Barstow--many large cracks in interior dry walls.

Inyokern--many large cracks in interior dry walls and plaster/stucco walls, few items were thrown from store shelves, few small objects fell, hanging objects or doors were swung moderately, hanging pictures were swung, buildings shook strongly, felt by all, awakened and frightened several.

Little Lake-few items were thrown from store shelves, many small objects were overturned and fell, hanging pictures fell, water sloshed in swimming pools, felt by all, awakened many and frightened all.

Ridgecrest--hairline cracks in interior plaster/stucco walls, felt by many, awakened and frightened few. Much merchandise was dumped from store shelves. One resident reported that bricks in his fireplace had shifted (press report).

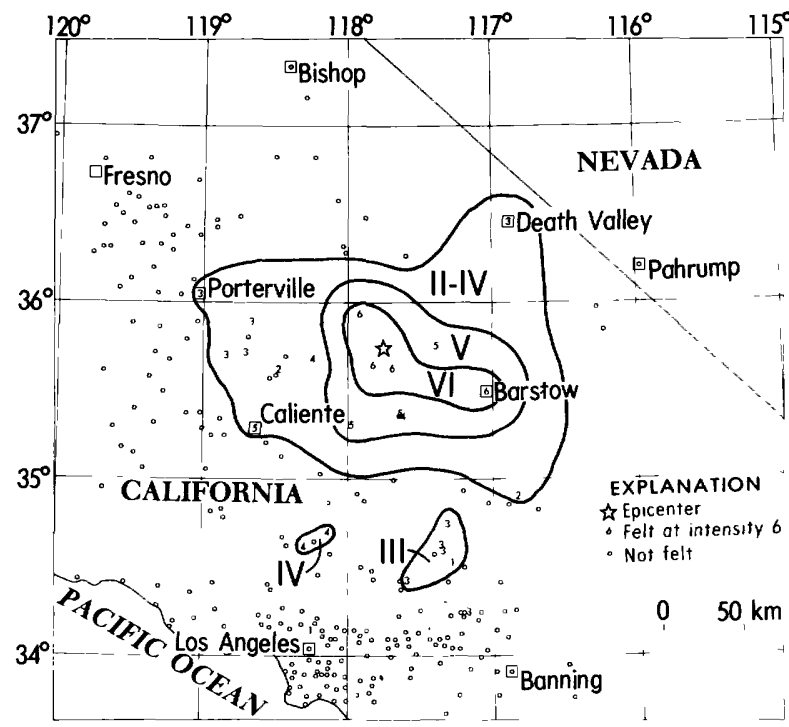

FIGURE 7.--Isoseismal map for the southern California earthquake of 01 October 1982, 142904.6 UTC. Roman numerals represent Modified Mercalli intensities between isoseismals; Arabic numerals are used to represent these intensities at specific sites.

Table 2.--Summary of macroseismic data for U. S. earthquakes. October-December 1982--Continued

\section{CALIFORNIA--Continued}

Intensity V:

Caliente--few cracked windows, few small objects were overturned and fell, few glassware or dishes were broken, hanging pictures were swung, felt by many, awakened and frightened few.

Cantil--hairline cracks in interior plaster/stucco walls, few small objects were overturned and fell, hanging pictures were swung out of place, building shook strongly, felt by several, awakened and frightened few.

Johannesburg--few items were thrown from store shelves, few small objects were overturned and fell, few glassware or dishes were broken, hanging pictures were swung out of place, felt by many, awakened few.

Trona--hairline cracks in interior plaster/stucco walls, water sloshed in swimming pools, light furniture or small appliances were overturned, few small objects overturned and fell, hanging pictures were swung out of place, hanging objects or doors were swung moderately, felt by all. 
Table 2.--Summary of macroseismic data for U. S. earthquakes, Octoher-December 1982--Continued

CALIFORNIA--Continued

Intensity IV: Lancaster, Leona Valley, Onyx, Red Mountain.

Intensity III: California Hot Springs (Pine Flat area), Death Valley, George Air Force Base, Glennville, Helendale, Oro Grande, Phelan, Pioneer Point, Porterville, Skyforest, Woody.

Intensity II: Mountain Mesa, Yermo.

Felt: Glendale, Goldstone Tracking Station, and Victorville (P).

2 October (B) Mammoth Lakes area

Origin time: 115215.6

Epicenter: $\quad 37.47 \mathrm{~N}$., $118.83 \mathrm{~W}$.

Depth: $\quad 5 \mathrm{~km}$

Magnitude: $\quad 3.7 \mathrm{ML}(\mathrm{B})$

Felt at Mammoth Lakes (B).

14 October (P) Southern California

Origin time: 005132.3

Epicenter: $\quad 34.20$ N., 118.65 W.

Depth: $\quad 2 \mathrm{~km}$

Magnitude: $\quad 2.2 \mathrm{ML}(\mathrm{P})$

Felt at Canoga Park (P).

14 October (B) Mammoth Lakes area

Origin time: 161131.5

Epicenter: $\quad 37.48 \mathrm{~N} ., 118.84 \mathrm{~W}$.

Depth: $\quad 8 \mathrm{~km}$

Magnitude: $\quad 3.7 \mathrm{ML}(\mathrm{P}), 3.9 \mathrm{ML}(\mathrm{B})$

Felt at Mammoth Lakes (B).

14 October (B) Mammoth Lakes area

Origin time: 193450.5

Epicenter: $\quad 37.49$ N., $118.82 \mathrm{~W}$.

Depth: $\quad 14 \mathrm{~km}$

Magnitude: $\quad 3.9 \mathrm{ML}(\mathrm{B}), 3.9 \mathrm{ML}(\mathrm{P})$

Felt at Mammoth Lakes (B).

15 October (P) Southern California

Origin time: 095720.5

Epicenter: $\quad 34.20$ N., 118.65 W.

Depth: $\quad 4 \mathrm{~km}$

Magnitude: $\quad 3.3 \mathrm{ML}(\mathrm{P})$

Intensity $\mathrm{V}$ :

Simi Valley--few items were thrown from store shelves, few small objects were overturned and fell, felt by and awakened many, frightened several.

Intensity IV: Canoga Park, Chatsworth, Northridge, Pacoima.

Felt: Malibu, Reseda, Van Nuys, Westwood

(press report).
Table 2. --Summary of macroseismic data for U. S. earthquakes, October-December 1982--Continued
16 October (B) Mammoth Lakes area

Origin time: 125413.9

Epicenter: $\quad 37.45 \mathrm{~N} ., 118.84 \mathrm{~W}$.

Depth: $\quad 7 \mathrm{~km}$

Magnitude: $\quad 3.9 \mathrm{ML}(\mathrm{B}), 3.9 \mathrm{ML}(\mathrm{P})$

Intensity IV: Bishop.

Felt: Mammoth Lakes (B).

19 October (P) Southern California

Origin time: 004942.2

Epicenter: $\quad 35.52 \mathrm{~N} ., 119.12 \mathrm{~W}$.

Depth: $\quad 6 \mathrm{~km}$

Magnitude: $\quad 3.6 \mathrm{ML}(\mathrm{P})$

Felt at Bakersfield (P).

19 October (B) Central California

Origin time: 220400.8

Epicenter: $\quad 37.03$ N., 121.74 W.

Depth: $\quad 11 \mathrm{~km}$

Magnitude: $\quad 3.7 \mathrm{ML}(\mathrm{B})$

This earthquake was felt throughout the San Francisco Bay area from Marin City to Monterey (B).

Intensity IV: Aromas, Morgan Hill.

Intensity III: Aptos, Castroville, Freedom, Santa Clara, Watsonville.

Felt: Gilroy and San Martin (B).

24 0ctober (P) Southern California

Origin time: 192319.0

Epicenter: $\quad 34.08 \mathrm{~N} ., 119.22 \mathrm{~W}$.

Depth: $\quad 14 \mathrm{~km}$

Magnitude: $\quad 3.7 \mathrm{ML}(\mathrm{P}), 3.8 \mathrm{ML}(\mathrm{B})$

Intensity $\mathrm{V}$ :

Saticoy-few windows were cracked, few small objects were overturned and fell, few glassware or dishes were broken, hanging pictures were swung, felt by many, awakened and frightened few.

Ventura--few windows were cracked, felt by many, frightened several.

Intensity IV: Oxnard. Felt: Point Mugu (P).

25 October (B) Central California

Origin time: 222604.3

Epicenter: $\quad 36.33 \mathrm{~N} ., 120.50 \mathrm{~W}$.

Depth: $\quad 11 \mathrm{~km}$

Magnitude: $\quad 5.3 \mathrm{mb}(\mathrm{G}), 5.2 \mathrm{MS}(\mathrm{G}), 5.4 \mathrm{ML}(\mathrm{B})$, $5.4 \mathrm{ML}(\mathrm{P})$

This earthquake was felt over an area of about $92,800 \mathrm{~km}^{2}$ of central California (fig. 8). The earthquake was felt in at least 14 counties--from Kern County on the 


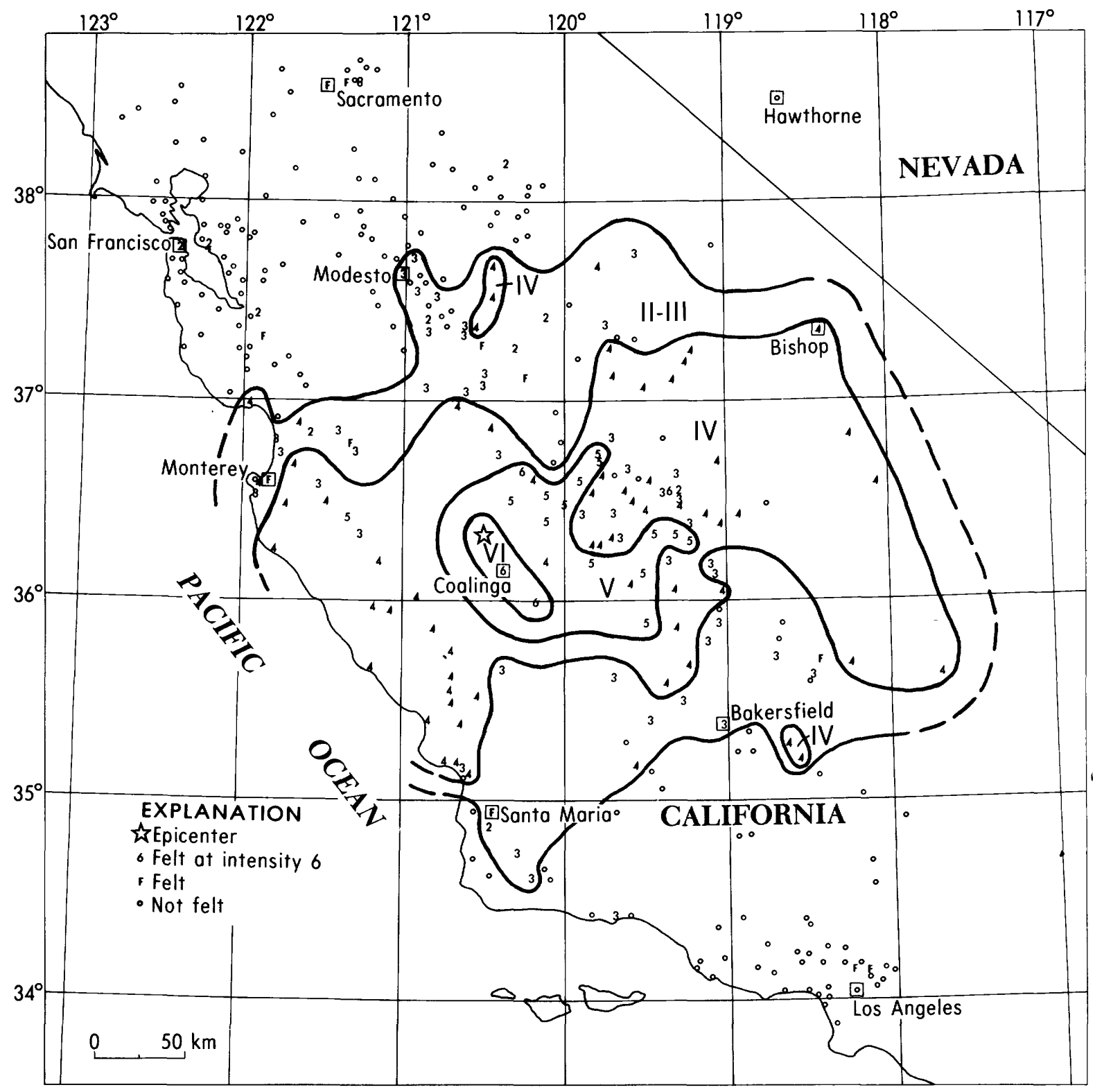

FIGURE 8.--Isoseismal map for the central California earthquake of 25 October 1982 , 222604.3 UTC. Roman numerals represent Modified Mercalli intensities between isoseismals; Arabic numerals are used to represent these intensities at specific sites.

south to Santa Clara County on the north and from coastal San Luis obispo County to Mono County on the eastern slope of the Sierra Nevada (press report).

At Coalinga, Lt. McDaniel of the state Highway Parol said, "when the trembling started, we got out of the building.... telephone poles and wires were swaying." He also reported that several stores were closed due to fallen merchandise blocking the aisles (press report).
A radio announcer for KOLI in Coalinga described the earth motion as "like a big jackhammer--up and down" (press report).

A hunter, 25 miles northwest of Coalinga in the coastal mountain range (between San Lucas and Priest City), gave the following account of the earthquake. "I was sitting on a steep hillside when the rock I was sitting on began moving. I immediately jumped up. I was nearly thrown off balance. I remember reaching down to hold 
Table 2.--Summary of macroseismic data for U. S. earthquakes, October-December 1982-.Continued

CALIFORNIA--Continued
Table 2.--Summary of macroseismic data for U. S. earthquakes, October-December 1982--Continued

CALIFORNIA--Cont inued onto the rock to keep from falling. The trees around me shook hard enough to knock the rainwater off their leaves. As sudden $1 y$ as it began, it stopped, lasting no longer than 5-7 seconds. I don't remember feeling any aftershocks. The next day $I$ noticed several areas where the ground had been cracked and lifted as much as five inches. Al1 over, even on the hilltops, small cracks could be seen in the wet ground."

Intensity VI:

Avenal--smal1 amounts of plaster fell from ceiling, some cracks in interior plaster walls, few items were thrown from store shelves, building shook strongly, a stationary 5-ton truck rocked slightly, felt by all, frightened few.

Coalinga--tiles fell from interior walls, many items were thrown from store shelves, hanging pictures were swung out of place, building shook strongly, observer experienced difficulty in standing or walking, hanging objects or doors were swung moderately, few small objects overturned and fell, felt by and frightened all.

Sultana--foundation was cracked, felt by many, frightened few.

Tranquillity--interior walls were split, hairline cracks in plaster/stucco walls, building shook strongly, felt by many, frightened few.

Intensity $\mathrm{V}$ :

Alpaugh--hairline cracks in plaster/stucco and dry walls, hanging pictures were swung, building shook strongly, felt by and frightened all.

Burrel--hairline cracks in interior dry wall and plaster/stucco walls, moving vehicles were rocked slightly, few items were thrown from store shelves, hanging pictures were swung out of place, few small objects were overturned and fell, few glassware or dishes were broken, light furniture or appliances were overturned, felt by many, frightened few.

Calwa--hairline cracks in plaster/stucco and drywa11, felt by many.

Cantua Creek--moving vehicles we re rocked moderately, few small objects were overturned, building shook strongly, observer experienced difficulty in standing or walking, felt by and frightened many.

Farmersville--moving vehicles we re rocked slightly, few items were thrown from store shelves, few small objects were overturned, felt by many.

Five Points--hairline cracks in plaster or stucco interior walls, few windows were cracked, hanging objects or doors were swung moderately, hanging pictures were swung, felt by all, frightened few.

Fresno--hairline cracks in interior plaster/stucco and dry walls, few windows were cracked, few items were thrown from store shelves, few small objects were overturned and fell, few glassware or dishes were broken, hanging pictures were swung, felt by many, frightened several, awakened few.

Goshen--few windows were cracked, felt by and frightened several.

Helm--many small objects were overturned and fell, pictures were swung, felt by and frightened many.

Raisin-hanging objects or doors were swung moderately, observer experienced difficulty in standing or walking, felt by and frightened several.

Soledad--few sma11 objects were overturned and fell, few glassware or dishes were broken, felt by and frightened several.

Stratford--few small objects were overturned and fell, hanging pictures were swung, hanging objects or doors were swung moderately, building shook strongly, felt by and frightened many.

Visalia--water splashed onto sides of swimming pools, lakes or ponds; building shook stronly; few small objects were overturned and fell; hanging pictures were swung; felt by many; frightened severa1.

Waukena--hairline cracks in interior dry wa11s; water splashed onto sides of swimming pools, lakes or ponds; few windows were cracked; few small objects were overturned and fell; few glassware or dishes were broken; hanging objects or doors were swung; felt by many; frightened few.

Intensity IV: Armona (hanging pictures were swung out of place), Aromas, Arroyo Grande, Atascadero, Auberry (hanging pictures were swung out of place), Avila Beach, Big Creek, Big Sur, Bishop (hanging pictures were swung), Bradley, Caliente, Capitola, Carmel Valley, Caruthers, Castle $\mathrm{AFB}$, Coarsegold, Corcoran, Creston (hanging pictures were swung), Dos Palos, Earlimart, Easton (hanging pictures were swung), E1 Portal (hanging pictures were swung), Fellows, Firebaugh, Fort Hunter 
Table 2.--Summary of macroseismic data for U. S. earthquakes. October-December 1982--Continued

CALIFORNIA--Continued

Liggett, Gonzales, Huron, Independence, Keene, King City, Kingsburg, La Grange (hanging pictures were swung), Lakeshore, Lemoncove, Lemoore (hanging pictures were swung), Lemoore--Naval Air Station (telephone service interrupted), Lockwood (hanging pictures were swung), Lone Pine (hanging pictures were swung), McFarland, Miramonte, Monterey (hanging pictures were swung), Morro Bay, O'Neals, Onyx, Paso Robles, Porterville (hanging pictures were swung), Prather, Reedley, Ridgecrest (hairline cracks in interior plaster/stucco walls, hanging picures were swung), Salinas, San Ardo (hanging pictures were swung), San Joaquin, San Luis Obispo (press report), San Miguel (hanging pictures were swung out of place), San Simeon, Santa Margarita (hanging pictures were swung), Selma, Shaver Lake, Snelling (shelves in a mobil home with ceramics were pulled away from wall causing few glassware/dishes to be broken), Templeton (hanging pictures were swung), Three Rivers, Tipton (hanging pictures were swung), Traver, Yettem, Wasco, Woodlake. Intensity III: Ahwanee, Bakersfield (hanging pictures were swung), Buellton, Buttonwillow, Carmel, Castroville, Chualar, Clovis, Cutler, Del Rey, Denair, Dinuba, Ducor (hanging pictures were swung), E1 Nido, Glennville, Greenfield, Hanford, Hollister, Ivanhoe, Jolon, Keyes, Lake Isabe11a (hanging pictures were swung), Laton, Lindsay, Los Alamos (hanging pictures were swung), Los Banos, Lost Hills, Mendota, Modesto, Moss Landing, Orange Cove, Paicines, Pismo Beach (hanging pictures we re swung), Red Top, Richgrove, Riverbank, Riverdale, Santa Rita Park (hanging pictures were swung), Shafter, Shandon, Soque1, Stevinson, Strathmore, Tulare, Ventura, Winton, Yosemite National Park. Intensity II: Alameda, Avery, Catheys Valley, Hilmar, Milpitas, Orcutt, Orosi, Planada, San Francisco, San Juan Bautista.

Fe1t: Atwater, Chowchilla, Glendale (P), Merced, Monterey ( $P$ ), Pasadena (P), Sacramento (P), San Jose (P), Santa Maria (P), Seaside, Tres Pinos, Wofford Heights.

25 October (B) Central California

Origin time: 231218.0

Epicenter: $\quad 36.34$ N., 120.51 W.

Depth: $\quad 12 \mathrm{~km}$

Magnitude: $\quad 4.3 \mathrm{mb}(\mathrm{G}), 4.2 \mathrm{ML}(\mathrm{P}), 4.3 \mathrm{ML}(\mathrm{B})$

Felt in the Coalinga-Hanford area (B).
Table 2.--Summary of macroseismic data for U. S. earthquakes, October-December 1982--Continued
25 October (B) Central California

Origin time: $231554, .5$

Epicenter: $\quad 36.34$ N., 120.50 W.

Depth: $\quad 11 \mathrm{~km}$

Magnitude: $\quad 4.0 \mathrm{ML}(\mathrm{P}), \quad 4.0 \mathrm{ML}(\mathrm{B})$

Felt in the Coalinga-Hanford area (B).

27 October (P) Southern California

Origin time: 102141.7

Epicenter: $\quad 33.88 \mathrm{~N} ., 118.22 \mathrm{~W}$.

Depth: $\quad 15 \mathrm{~km}$

Magnitude: $\quad \cdot 2.9 \mathrm{ML}(\mathrm{P})$

This earthquake was felt in portions of southern Los Angeles County (press report), including Gardena ( $P$ ) and Huntington Park.

28 October (P) Southern California

Origin time: 094036.3

Epicenter: $\quad 33.83$ N., $117.10 \mathrm{~W}$.

Depth: $\quad 17 \mathrm{~km}$

Magnitude: $2.9 \mathrm{ML}(\mathrm{P})$

Felt at Riverside (P).

4 November (B) Northern California

Origin time: 151041.3

Epicenter: $\quad 38.44$ N., 122.27 W.

Depth: $\quad 14 \mathrm{~km}$

Magnitude: $\quad 3.2 \mathrm{ML}(\mathrm{B})$

Felt in Napa County (press report).

4 November (P) Southern California

Origin time: 161340.7

Epicenter: $\quad 33.88 \mathrm{~N} ., 117.92 \mathrm{~W}$.

Depth: $\quad 6 \mathrm{~km}$

Magnitude: $\quad 2.8 \mathrm{ML}(\mathrm{P})$

Felt at Anaheim, Buena Park, and Fullerton (P).

9 November (B) Northern California

Origin time: 111205.8

Epicenter: $\quad 39.74$ N., $120.60 \mathrm{~W}$.

Depth: $\quad 10 \mathrm{~km}$

Magnitude: $\quad 3.0 \mathrm{ML}(\mathrm{B})$

Intensity IV: Blairsden, Portola.

10 November ( $P$ ) Southern California

Origin time: 112125.7

Epicenter: $\quad 34.05 \mathrm{~N} ., 116.67 \mathrm{~W}$.

Depth:

Magnitude: $\quad 4.1 \mathrm{mb}(\mathrm{G}), 4.4 \mathrm{ML}(\mathrm{B}), 3.9 \mathrm{ML}(\mathrm{P})$

Intensity $\mathrm{V}$ :

Big Bear Lake-few windows were cracked, hanging pictures were swung out of 
Table 2.--Summary of macroseismic data for U. S. earthquakes. October-December 1982--Continued

place, few small objects were overturned and fell, few glassware or dishes were broken.

Desert Hot Springs--few items were thrown from store shelves, few small objects were overturned, felt by many, awakened and frightened several.

Idy11wild--few small objects fel1; felt by, awakened, and frightened several.

White Water--hanging pictures fell; few small objects were overturned and fel1; building shook slightly to strongly; hanging objects or doors were swung moderately; felt by, awakened, and frightened many.

Intensity IV: Cabazon, Forest Falls, Mecca, Marongo Valley.

Intensity III...: Banning, Indio, Landers, North Palm Springs, Thousand Palms.

10 November (B) Mammoth Lakes area

Origin time: 132957.4

Epicenter: $\quad 37.48 \mathrm{~N} \cdot, 118.82 \mathrm{~W}$.

Depth: $\quad 5 \mathrm{~km}$

Magnitude: $\quad 3.6 \mathrm{ML}(\mathrm{B})$

Felt at Mammoth Lakes.

12 November (P) Imperial Valley

Origin time: 093355.2

Epicenter: $\quad 32.98 \mathrm{~N} ., 115.57 \mathrm{~W}$.

Depth: $\quad 14 \mathrm{~km}$

Magnitude: $2.6 \mathrm{ML}(\mathrm{P})$

Felt in the Imperial Valley (P).

12 November (B) Central California

Origin time: 215711.9

Epicenter: $\quad 35.33$ N., 120.54 W.

Depth: $\quad 9 \mathrm{~km}$

Magnitude: $\quad 3.4 \mathrm{ML}(\mathrm{P}), 3.0 \mathrm{ML}(\mathrm{B})$

Felt at San Luis Obispo (B).

13 November (B) Central California

Origin time: 201820.5

Epicenter: $\quad 36.69 \mathrm{~N} ., 121.20 \mathrm{~W}$.

Depth: $\quad 9 \mathrm{~km}$

Magnitude: $\quad 3.7 \mathrm{ML}(\mathrm{B})$

Intensity III: Prunedale, Salinas. Felt: Hollister (B)

26 November (B) Northern California Origin time: 092949.1

Epicenter: $\quad 38.28 \mathrm{~N} ., 122.16 \mathrm{~W}$.

Depth: $\quad 11 \mathrm{~km}$

Magnitude: $\quad 3.3 \mathrm{ML}(\mathrm{B})$
Table 2.--Summary of macroseismic data for U. S. earthquakes, October-December 1982--Continued

\section{CALIFORNIA--Cont inued}

Intensity IV: Napa .

Felt: Fairfield (B), Martinez (press report).

26 November (P) Southern California

Origin time: 123013.8

Epicenter: $\quad 34.84 \mathrm{~N} \cdot, 118.96 \mathrm{~W}$.

Depth: $\quad 5 \mathrm{~km}$

Magnitude: $3.1 \mathrm{ML}(\mathrm{P})$

Intensity IV: Frazier Park.

Intensity III: Lebec.

Intensity II: Ontario.

28 November (B) Mammoth Lakes area -

origin time: $\quad 144319.1$

Epicenter: $\quad 37.44 \mathrm{~N}, 118.85 \mathrm{~W}$.

Depth: $\quad 3 \mathrm{~km}$

Magnitude: $\quad 3.5 \mathrm{ML}(\mathrm{B})$

Felt at Mammoth Lakes.

2 December (B) Northern California

Oriin time: $\quad 160245.9$

Epicenter: $\quad 39.17 \mathrm{~N} ., 122.21 \mathrm{~W}$.

Depth: $\quad 10 \mathrm{~km}$

Magnitude: $\quad 3.0 \mathrm{ML}(\mathrm{B})$

Felt in the Lake Berryessa and Clear Lake areas $(B)$.

4 December Southern California

Origin time: 0308

Epicenter: Not located

Depth: Not computed

Magnitude: $2.2 \mathrm{ML}(\mathrm{P})$

Intensity III: Inglewood (press report).

7 December (B) Central California

Origin time: 230539.1

Epicenter: $\quad 36.92 \mathrm{~N}, 121.70 \mathrm{~W}$.

Depth: $\quad 10 \mathrm{~km}$

Magnitude: $3.1 \mathrm{ML}(\mathrm{B})$

Felt at Watsonville (B).

12 December (B) Mammoth Lakes area

Origin time: 103806.0

Epicenter: $\quad 37.54 \mathrm{~N} ., 118.82 \mathrm{~W}$.

Depth: $\quad 6 \mathrm{~km}$

Magnitude: $\quad 3.5 \mathrm{ML}(\mathrm{P}), 3.6 \mathrm{ML}(\mathrm{B})$

Felt at Mammoth Lakes (B).

12 December (B) Mammoth Lakes area

Origin time: 205908.8

Epicenter: $\quad 37.53 \mathrm{~N} ., 118.81 \mathrm{~W}$. 


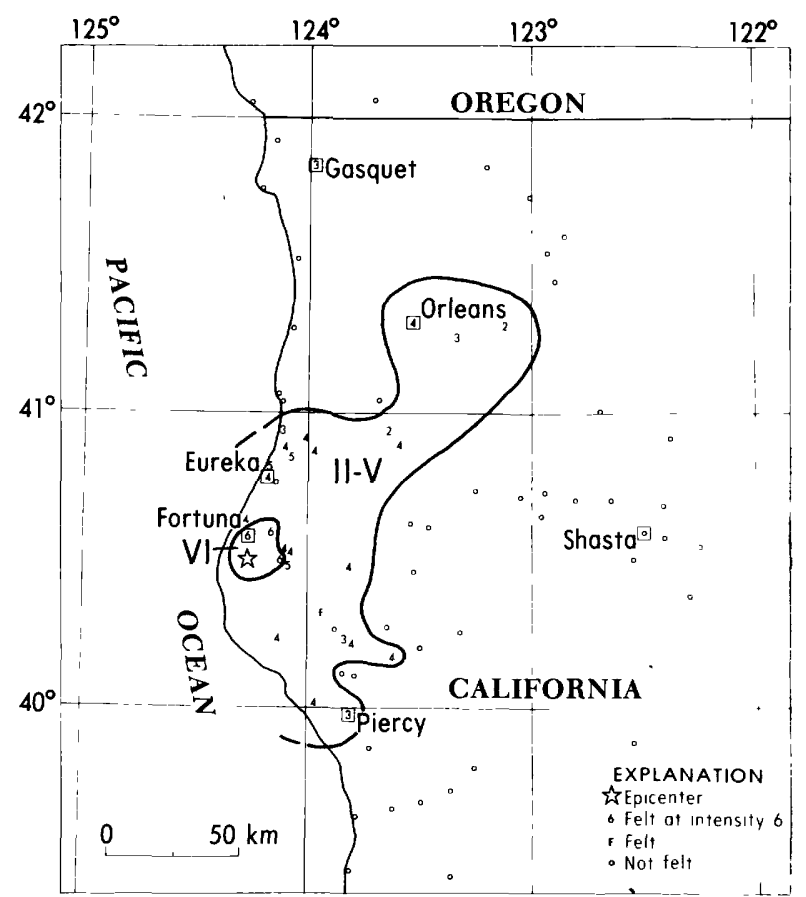

FIGURE 9.--Isoseismal map for the northern California earthquake of 16 December 1982, 065301.3 UTC. Roman numerals represent Modified Mercalli intensities between isoseismals; Arabic numerals are used to represent these intensities at specific sites.

Table 2.--Summary of macroseismic data for U. S. earthquakes, October-December 1982--Continued

CALIFORNIA--Continued

Depth: $\quad 4 \mathrm{~km}$

Magnitude: $\quad 3.8 \mathrm{ML}(\mathrm{P}), 3.7 \mathrm{ML}(\mathrm{B})$

Felt at Mammoth Lakes (B).

14 December (B) Central California

Origin time: 064609.9

Epicenter: $\quad 36.91 \mathrm{~N} ., 121.49 \mathrm{~W}$.

Depth: $\quad 5 \mathrm{~km}$

Magnitude: $\quad 3.2 \mathrm{ML}(\mathrm{B})$

Felt at Hollister (B).

14 December (B) Northern California

Origin time: 191531.8

Epicenter: $\quad 40.54$ N., 124.20 W.

Depth: $\quad 20 \mathrm{~km}$

Magnitude: $\quad 3.6 \mathrm{ML}(\mathrm{B})$

Felt at Ferndale (B).

16 December (B) Northern California

Origin time: 065301.3
Table 2.--Summary of macroseismic data for U. S. earthquakes, October-December 1982--Continued

CALIFORNIA--Continued

Epicenter: $\quad 40.50 \mathrm{~N} ., 124.26 \mathrm{~W}$.

Depth: $\quad 18 \mathrm{~km}$

Magnitude: $\quad 4.8 \mathrm{mb}(\mathrm{G}), 4.5 \mathrm{MS}(\mathrm{G}), 4.4 \mathrm{ML}(\mathrm{B})$

This earthquake was felt over an area of about $8,200 \mathrm{~km}^{2}$ of northern California (fig. 9).

The Fortuna sheriff dispatcher gave the following account of the earthquake: "It wasn't one of the nice, gentle rollers. It was like a big bang. It shook for about 45 seconds. Then there was another, like an aftershock, for about 20 seconds, a rumbling" (press report).

Residents in Ferndale reported windows shattered, chimneys cracked, and pictures were knocked off the walls in the restored section of Victorian homes and businesses.

In Fortuna, stores had broken windows and grocery stores had cans knocked from shelves (press report).

Intensity VI:

Ferndale--chimneys were cracked, windows were shattered, pictures were knocked from walls (press report).

Fortuna--chimneys we re cracked, plate glass windows were broken or shattered, much merchandise was knocked from store shelves (press report).

Rio Dell--few items were thrown from store shelves; hanging pictures were swung out of place with some fallen; some windows were broken; few small objects were overturned, fell, and were broken; light furniture or small appliances were overturned; few glassware or dishes were broken; hanging objects or doors were swung moderately; felt by all.

Intensity $\mathrm{V}$ :

Bayside--moving vehicles were rocked slightly, few small objects were overturned and fell, few glassware or dishes were broken.

Samoa--few items were thrown from store shelves, few small objects were overturned and fell.

Scotia--few small objects were overturned and fell, hanging doors or objects were swung moderately.

Intensity IV: Alderpoint, Arcata, Blue Lake, Bridgeville, Carlotta, Eureka, Honeydew, Hydesville, Korbel, Loleta, Orleans, Phillipsville, Salyer, Whitethorn. 
Table 2.--Summary of macroseismic data for U. S. earthquakes, October-December 1982--Continued

CALIFORNIA--Continued

Intensity III: Forks of Salmon, Gasquet, McKinleyville, Miranda, Piercy, Swains Flat.

Intensity II: Sawyers Bar, Willow Creek. Felt: Weott.

20 December (B) Northern California

Origin time: 000821.1

Epicenter: $\quad 40.54$ N., 123.97 W.

Depth: $\quad 5 \mathrm{~km}$

Magnitude: $\quad 3.3 \mathrm{ML}(\mathrm{B})$

Felt ain the Eureka area (B).

21 December Northern California

Origin time: 0933

Epicenter: Not located

Depth: None computed

Magnitude: $2.3 \mathrm{ML}(\mathrm{B})$

Intensity III: Clearlake Highlands (press report).

21 December (B) Mammoth Lakes area

Origin time: 222812.9

Epicenter: $\quad 37.63 \mathrm{~N} ., 118.96 \mathrm{~W}$.

Depth: $\quad 5 \mathrm{~km}$

Magnitude: $\quad 3.7 \mathrm{ML}(\mathrm{P}), 3.3 \mathrm{ML}(\mathrm{B})$

Felt at Mammoth Lakes (B).

22 December (B) Owens Valley area

Origin time: 094049.9

Epicenter: $\quad 37.36 \mathrm{~N} ., 118.52 \mathrm{~W}$.

Depth: $\quad 10 \mathrm{~km}$

Magnitude: $\quad 3.4 \mathrm{ML}(\mathrm{P}), 3.4 \mathrm{ML}(\mathrm{B})$

Felt in the Bishop area (B).

22 December (P) Southern California

Origin time: $\quad 144736.9$

Epicenter: $\quad 35.75 \mathrm{~N} ., 117.75 \mathrm{~W}$.

Depth: $\quad 10 \mathrm{~km}$

Magnitude: $\quad 3.8 \mathrm{ML}(\mathrm{B}), 3.3 \mathrm{ML}(\mathrm{P})$

Felt at Ridgecrest (P).

26 December (B) Northern California

origin time: 095946.6

Epicenter: $\quad 38.81 \mathrm{~N} ., 122.78 \mathrm{~W}$.

Depth: $\quad 1 \mathrm{~km}$

Magnitude: $\quad 3.1 \mathrm{ML}(\mathrm{B})$

Intensity IV: Cobb (press report).

26 December (B) Central California

Origin time: 100424.9

Epicenter: $\quad 36.81 \mathrm{~N} ., 121.55 \mathrm{~W}$.
Table 2.--Summary of macroseismic data for U. S. earthquakes, October-December 1982-.Continued

\section{CALIFORNIA--Continued}

$\begin{array}{ll}\text { Depth: } & 6 \mathrm{~km} \\ \text { Magnitude: } & 3.5 \mathrm{ML}(\mathrm{B})\end{array}$

Felt at Hollister and San Juan Bautista (B).

28 December (B) Mammoth Lakes area

Origin time: 004938.8

Epicenter: $\quad 37.49 \mathrm{~N} ., 118.80 \mathrm{~W}$.

Depth: $\quad 3 \mathrm{~km}$

Magnitude: $\quad 3.8 \mathrm{ML}(\mathrm{P}), 3.6 \mathrm{ML}(\mathrm{B})$

Felt at Mammoth Lakes (B).

28 December (B) California-Nevada border region Origin time: 190624.0

See Nevada listing.

30 December (P) Southern California

Origin time: 040029.5

Epicenter: $\quad 33.95$ N., $118.82 \mathrm{~W}$.

Depth: $\quad 0 \mathrm{~km}$

Magnitude: $\quad 3.6 \mathrm{ML}(\mathrm{P}), 4.0 \mathrm{mb}(\mathrm{G})$

Intensity III: Malibu (press report).

31 December (P) Southern California

Origin time: 090723.3

Epicenter: $\quad 35.82 \mathrm{~N} ., 117.73 \mathrm{~W}$.

Depth: $\quad 6 \mathrm{~km}$

Magnitude: $\quad 4.4 \mathrm{mb}(G), 4.5 \mathrm{ML}(\mathrm{B}), 4.0 \mathrm{ML}(\mathrm{P})$

Intensity IV: Trona.

CALIFORNIA--Off the Coast

4 December (B) Northern California

Origin tíme: 030823.2

Epicenter: $\quad 40.30 \mathrm{~N} ., 124.52 \mathrm{~W}$.

Depth: $24 \mathrm{~km}$

Magnitude: $3.1 \mathrm{ML}(\mathrm{B})$

Felt at Honeydew and Petrolia (B).

\section{COLORADO}

22 November (G) Northwestern Colorado

Origin time: 100901.4

Epicenter: $\quad 39.74$ N., $107.58 \mathrm{~W}$.

Depth: $\quad 5 \mathrm{~km}$

Magnitude: $2.9 \mathrm{ML}(G)$

Felt at the Rifle Fish Hatchery about 15 miles northeast of Rifle (telephone report). 
Table 2.--Summary of macroseismic data for U. S. earthquakes, October-December 1982--Continued

GEORGIA

31 October (G) Western Georgia

Origin time: 030739.4

Epicenter: $\quad 32.66 \mathrm{~N}, 84.91 \mathrm{~W}$.

Depth: $\quad 5 \mathrm{~km}$

Magnitude: $\quad 2.9 \mathrm{Mn}(\mathrm{G}), 2.9 \mathrm{MD}(\mathrm{K})$

This earthquake was felt in Harris and Muscogee Counties in Georgia and Russell County, Alabama. Many of the citizens described the earthquake sound as an explosion, a plane crash, or a train derailment (press report).

Intensity V:

Georgia--

Columbus (Beallwood)--few small objects fell, felt by many, awakened several, frightened few.

Elberslie--few small objects were overturned and fell, small amounts of plaster/stucco fell from interior walls, felt by many, awakened and frightened several.

Midland--few small objects were overturned and fell, few glassware or dishes were broken, felt by many, awakened and frightened several.

Intensity IV:

Alabama--Phenix City.

Georgia--Buena Vista, Cataula, Columbus

(Windsor Park), Upatoi.

Intensity III:

Alabama--Smiths.

Georgia--Columbus (Baker Village, Lindsay

Creek, Wynnton), Columbus Metropolitan

Airport, Hamilton.

31 October (G) Western Georgia

Origin time: 031215.6

Epicenter: $\quad 32.65 \mathrm{~N} ., 84.87 \mathrm{~W}$.

Depth: $\quad 5 \mathrm{~km}$

Magnitude: $\quad 3.1 \mathrm{Mn}(\mathrm{G})$

Felt in Harris and Muscogee Counties in Georgia and in the Phenix City, Alabama area (press report).

21 December (F) Central Georgia

Origin time: 053046.2

Epicenter: $\quad 32.80 \mathrm{~N} \cdot, 83.52 \mathrm{~W}$.

Depth: $\quad 0 \mathrm{~km}$

Magnitude: $2.7 \mathrm{MD}(\mathrm{F})$
Table 2.--Summary of macroseismic data for U. S. earthquakes, October-December 1982--Continued

GEORGIA--Continued

This is one of a swarm of 100 earthquakes recorded in this area from December 1982 to March 1983.

Intensity III: Macon.

HAWAII

11 October (H) Island of Hawaii

Origin time: 185953.5

Epicenter: $\quad 19.38 \mathrm{~N} ., 155.25 \mathrm{~W}$.

Depth: $\quad 3 \mathrm{~km}$

Magnitude: $\quad 3.1 \mathrm{ML}(\mathrm{H})$

Intensity III: Hawailan Volcano Observatory.

22 October (H) Island of Hawaii

Origin time: 131329.0

Epicenter: $\quad 19.33 \mathrm{~N} ., 155.19 \mathrm{~W}$.

Depth: $\quad 10 \mathrm{~km}$

Magnitude: $\quad 3.3 \mathrm{ML}(\mathrm{H})$

Intensity II: Hilo, Mauna Kea Observatory.

23 October (H) Island of Hawaii

Origin time: 074256.4

Epicenter: $\quad 19.35$ N., $155.22 \mathrm{~W}$.

Depth: $\quad 9 \mathrm{~km}$

Magnitude: $\quad 3.0 \mathrm{ML}(\mathrm{H})$

Intensity II: Volcano.

25 October (H) Island of Hawaii

Origin time: 161930.6

Epicenter: $\quad 19.33$ N., 155.19 W.

Depth: $\quad 10 \mathrm{~km}$

Magnitude: $3.2 \mathrm{ML}(\mathrm{H})$

Intensity III: Hilo, Volcano.

1 November (H) Island of Hawaii

Origin time: 022445.7

Epicenter: $\quad 19.33$ N., 155.12 W.

Depth: $8 \mathrm{~km}$

Magnitude: $\quad 3.0 \mathrm{ML}(\mathrm{H})$

Intensity II: Puu Oo.

13 November (H) Island of Hawaii

origin time: 021858.2

Epicenter: $\quad 19.45 \mathrm{~N} ., 155.44 \mathrm{~W}$.

Depth: $\quad 15 \mathrm{~km}$

Magnitude: $\quad 4.1 \mathrm{ML}(\mathrm{H}), 4.4 \mathrm{mb}(\mathrm{G})$

Intensity $\mathrm{V}$ : Pahala.

Intensity IV: Hilo, Kurtistown, Volcano.

Intensity III: Kamuela, Kealakekua, Paauilo. 
Table 2.-Summary of macroseismic data for $U$. S. earthquakes, October-December 1982--Continued

HAWA I I--Continued

13 November (H) Island of Hawaii

Origin time: 022829.0

Epicenter: $\quad 19.46 \mathrm{~N}$, $155.43 \mathrm{~W}$.

Depth: $\quad 15 \mathrm{~km}$

Magnitude: $3.3 \mathrm{ML}(\mathrm{H})$

Intensity III: Pahala.

25 November ( $H$ ) Island of Hawaii

Origin time: 191037.3

Epicenter: $\quad 19.36 \mathrm{~N} ., 155.30 \mathrm{~W}$.

Depth: $\quad 35 \mathrm{~km}$

Magnitude: $\quad 3.6 \mathrm{ML}(\mathrm{H})$

Intensity III: Hilo, Pahala.

28 November (H) Island of Hawaii

Origin time: 020125.5

Epicenter: $\quad 19.28 \mathrm{~N}$., $155.51 \mathrm{~W}$.

Depth: $\quad 9 \mathrm{~km}$

Magnitude: $\quad 3.8 \mathrm{ML}(\mathrm{H})$

Intensity IV: Pahala.

30 November (H) Island of Hawaii

Origin time: 005038.0

Epicenter: $\quad 19.35 \mathrm{~N} ., 155.40 \mathrm{~W}$.

Depth: $\quad 32 \mathrm{~km}$

Magnitude: $\quad 4.0 \mathrm{ML}(\mathrm{H})$

Intensity IV: Glenwood, Hilo, Kalapana, Mountain View, Pahala, Volcano.

Intensity III: Hawaiian Ocean View Estates Papaikou, Pepeekeo.

5 December (H) Island of Hawaii

Origin time: 153737.7

Epicenter: $\quad 19.81 \mathrm{~N} ., 156.16 \mathrm{~W}$.

Depth: $39 \mathrm{~km}$

Magnitude: $\quad 3.8 \mathrm{ML}(\mathrm{H})$

Intensity III: Waikaloa.

10 December (H) Island of Hawaii

Origin time: 053811.7

Epicenter: $\quad 19.40$ N., 155.29 W.

Depth: $\quad 3 \mathrm{~km}$

Magnitude: $\quad 3.1 \mathrm{ML}(\mathrm{H})$

Intensity IV: Hawaii Volcanoes National Park. Intensity III : Volcano.

10 December (H) Island of Hawaii

Origin time: 060051.6

Epicenter: $\quad 19.39 \mathrm{~N} ., 155.24 \mathrm{~W}$.

Depth: $\quad 4 \mathrm{~km}$

Magnitude: $\quad 3.1 \mathrm{ML}(\mathrm{H})$

Intensity IV: Hawaii Volcanoes National Park. Intensity III: Volcano.

28 December (H) Island of Hawaii Origin time: 093501.9

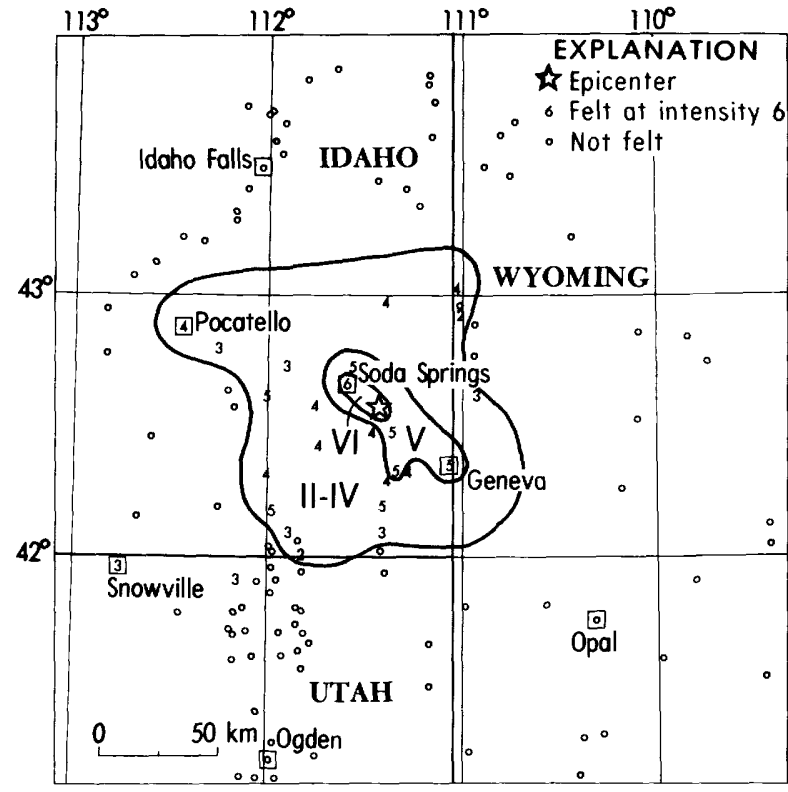

FIGURE 10.--Isoseismal map for the southeastern Idaho earthquake of 14 0ctober $1982,04 \quad 1024.3$ UTC. Roman numerals represent Modified Mercalli intensities between isoseismals; Arabic numerals are used to represent these intensities at

Table 2.--Summary of macroseismic data for U. S. earthquakes, October-December 1982--Continued

HAWA I I--Cont inued

Epicenter: $\quad 19.34$ N., $155.10 \mathrm{~W}$.

Depth: $\quad 9 \mathrm{~km}$

Magnitude: $\quad 3.2 \mathrm{ML}(\mathrm{H})$

Intensity II: Hilo.

31 December (H) Island of Hawaii

Origin time: 135510.0

Epicenter: $\quad 19.38$ N., 155.24 W.

Depth: $\quad 1 \mathrm{~km}$

Magnitude: $\quad 3.4 \mathrm{ML}(\mathrm{H})$

Intensity II: Volcano.

\section{IDAHO}

7 October (G) Southeastern Idaho

Origin time: 092602.6

Epicenter: $\quad 43.00 \mathrm{~N} ., 111.07 \mathrm{~W}$.

Depth: $\quad 5 \mathrm{~km}$

Magnitude: $\quad 3.0 \mathrm{ML}(\mathrm{G}), 3.5 \mathrm{MD}(\mathrm{D})$

Intensity IV:

Wyoming--Freedom .

Intensity II:

Wyoming--Thayne. 
Table 2.--Summary of macroseismic data for U. S. earthquakes, Octuber-December 1982--Continued
Table 2.--Summary of macroseismic data for U. S. earthquakes, October-December 1982--Continued

\section{IDAHO--Cont inued}

8 October (U) Southeastern Idaho

Origin time: 095332.1

Epicenter: $\quad 42.62 \mathrm{~N} ., 111.47 \mathrm{~W}$.

Depth: $\quad 7 \mathrm{~km}$

Magnitude: $3.5 \mathrm{ML}(\mathrm{U})$

Intensity IV:

Idaho--Dingle, Geneva, Georgetown, Soda Springs.

Intensity III:

Idaho--Bancrof $t$, Bloomington.

Wyoming--Etna, Freedom.

8 October (U) Southeastern Idaho

Origin time: 100659.0

Epicenter: $\quad 42.62$ N., 111.47 W.

Depth: $\quad 7 \mathrm{~km}$

Magnitude: $\quad 3.8 \mathrm{ML}(\mathrm{U}), 4.0 \mathrm{ML}(\mathrm{D})$

Intensity V:

Conda-few items were thrown from store shelves, few small objects were overturned and fell, building shook strongly, hanging pictures were,swung, felt by many.

Soda Springs--a few homes reported enlarged wall cracks.

8 October (U) Southeastern Idaho

Origin time: 160409.0

Epicenter: $\quad 42.63 \mathrm{~N} ., 111.49 \mathrm{~W}$.

Depth: $\quad 7 \mathrm{~km}$

Magnitude: $\quad 3.2 \mathrm{ML}(\mathrm{U})$

Felt at Soda Springs (press report).

14 October (U) Southeastern Idaho

Origin time: 041024.3

Epicenter: $\quad 42.59 \mathrm{~N} ., 111.43 \mathrm{~W}$

Depth: $\quad 7 \mathrm{~km}$

Magnitude: $\quad 4.6 \mathrm{mb}(\mathrm{G}), 4.7 \mathrm{ML}(\mathrm{U}), 4.9 \mathrm{ML}(\mathrm{D})$

This earthquake was felt over an area of about $13,500 \mathrm{~km}^{2}$ of Idaho, Utah, and Wyoming ( $f$ ig. 10). The earthquake was heard but not felt at Idaho Falls where the noise was described as sounding like a "sonic boom."

$\frac{\text { Intensity VI: }}{\text { Idaho-- }}$

Soda Springs--hairline cracks in interior dry walls, a crack in basement walls was enlarged, foundation was cracked, buildings shook slightly to strongly, hanging pictures were swung, hanging objects or doors were swung moderately, felt by many, awakened few, frightened several.

Soda Springs ( 4 miles southeast)--bricks

\section{IDAHO--Cont inued}

fell from chimneys and a few buildings were reported damaged.

Intensity $\mathrm{V}$ :

Idaho--

Bloomington--a stack of wood fell, few small objects fell, building shook slightly to strongly, felt by several.

clifton--hairline cracks in interior dry wall, felt by few, frightened few.

Conda--few items were thrown from store shelves, few small objects fell, felt by many, awakened few.

Geneva--few small objects were overturned and fell, hanging pictures were swung, felt by many, awakened several.

Georgetown--few items were thrown from store shelves; water sloshed in swimming pools, lakes, or ponds; few small objects overturned; hanging pictures were swung out of place; hanging objects or doors were swung moderately; buildings shook slightly to strongly; felt by all; awakened and frightened many.

Lava Hot Springs--few windows were cracked, few small objects were overturned and fell, few glassware or dishes were broken, hanging pictures were swung, felt by many.

Montpelier--few small objects were overturned and fell, hanging pictures were swung, felt by many, awakened few, frightened several.

Intensity IV:

Idaho--Dingle (pictures were swung out of place), Grace, Nounan (pictures were swung out of place), Ovid, Paris, Pocatello (few items were thrown from store shelves), Swan Lake, Thatcher, Wayan.

Wyoming--Etna (few small objects were overturned).

Intensity III:

Idaho--Bancroft, Inkom (building shook strongly), Preston, Saint Charles.

Utah--Plymouth, Snowville.

Wyoming--Smoot (water beds jiggled).

Intensity II:

Idaho-Franklin.

Wyoming--Thayne.

24 December (G) Southeastern Idaho

Origin time: 151120.1

Epicenter: $\quad 42.12 \mathrm{~N} ., 112.56 \mathrm{~W}$.

Depth: $\quad 5 \mathrm{~km}$

Magnitude: $\quad 3.5 \mathrm{ML}(\mathrm{U})$ 
Table 2.--Summary of macroseismic data for U. S. earthquakes, October-December 1982--Continued

Intensity III:

Idaho--Holbrook, Malad City, Stone.

Utah--Snowville.

\section{IOWA}

15 November (G) Southeastern South Dakota Origin time: 025822.9

See South Dakota listing.

\section{MAINE}

1 December $(\mathrm{J})$ Central New Hampshire

Origin time: 225223.0

See New Hampshire listing.

\section{MONTANA}

21 October (G) Hebgen Lake area

Origin time: 060528.2

Epicenter: $\quad 44.72 \mathrm{~N} ., 111.83 \mathrm{~W}$.

Depth: $\quad 5 \mathrm{~km}$

Magnitude: $\quad 4.4 \mathrm{MD}(\mathrm{D}), 4.4 \mathrm{ML}(\mathrm{G})$

Intensity IV: Belgrade.

Intensity III: Lima, Pony.

Felt: Livingston and Virginia City (telephone report).

26 October (G) Hebgen Lake area

Origin time: 082629.9

Epicenter: $\quad 44.75$ N., 111.75 W.

Depth: $\quad 5 \mathrm{~km}$

Magnitude: $\quad 4.3 \mathrm{MD}(\mathrm{D}), 4.6 \mathrm{ML}(\mathrm{G})$

Intensity IV: Virginia City.

Intensity III: Melrose, Twin Bridges.

4 November (G) Hebgen Lake area

Origin time: 095829.9

Epicenter: $\quad 44.72 \mathrm{~N} ., 111.72 \mathrm{~W}$.

Depth: $\quad 5 \mathrm{~km}$

Magnitude: $\quad 4.2 \mathrm{ML}(\mathrm{G}), 4.1 \mathrm{MD}(\mathrm{D})$

Intensity IV: Harrison.

\section{NEBRASKA}

15 November (G) Southeastern South Dakota Origin time: 025822.9

See South Dakota listing.

Table 2.--Summary of macroseismic data for U. S. earthquakes, October-December 1982--Continued

12 November (E) Southern Nevada Origin time: 191700.103

Epicenter: $\quad 37.02 \mathrm{~N} ., 116.03 \mathrm{~W}$.

Depth: $\quad 0 \mathrm{~km}$

Magnitude: $\quad 4.4 \mathrm{mb}(\mathrm{G}), 4.3 \mathrm{ML}(\mathrm{B})$

Nevada Test Site explosion "SEYVAL" at $37^{\circ} 01^{\prime} 25.21^{\prime \prime}$ N., $116^{\circ} 01^{\prime} 55.49^{\prime \prime}$ W., surface elevation $1214 \mathrm{~m}$, depth of burial $366 \mathrm{~m}$.

7 December (G) Southern Nevada

Origin time: 094349.6

Epicenter: $\quad 36.02 \mathrm{~N} ., 114.83 \mathrm{~W}$.

Depth: $\quad 5 \mathrm{~km}$

Magnitude: $\quad 3.3 \mathrm{ML}(\mathrm{G}), 3.7 \mathrm{ML}(\mathrm{P})$

Intensity II: Boulder City.

10 December (E) Southern Nevada

Origin time: 152000.090

Epicenter: $\quad 37.03 \mathrm{~N} ., 116.07 \mathrm{~W}$.

Depth: $\quad 0 \mathrm{~km}$

Magnitude: $\quad 4.6 \mathrm{mb}(\mathrm{G}), 4.7 \mathrm{ML}(\mathrm{B})$

Nevada Test Site explosion "MANTECA" at $37^{\circ} 01^{\prime} 48.66^{\prime \prime}$ N., $116^{\circ} 04^{\prime} 18.80^{\prime \prime}$ W., surface elevation $1263 \mathrm{~m}$, depth of burial $413 \mathrm{~m}$.

28 December (B) California-Nevada border region Origin time: 190624.8

Epicenter: $\quad 38.03$ N., $118.42 \mathrm{~W}$.

Depth: $\quad 8 \mathrm{~km}$

Magnitude: $\quad 4.7 \mathrm{mb}(\mathrm{G}), 5.2 \mathrm{ML}(\mathrm{P}), 4.9 \mathrm{ML}(\mathrm{B})$

Intensity IV:

California--Benton.

Intensity III:

California--Miramonte.

Nevada--Dyer, Mina, Schurz.

Felt :

California--Bishop and in the Mono Lake area (B).

\section{NEW HAMPSHIRE}

1 December ( $\mathrm{J}$ ) Central New Hampshire

Origin time: 225223.0

Epicenter: $\quad 43.61 \mathrm{~N} ., 71.49 \mathrm{~W}$.

Depth: $\quad 0 \mathrm{~km}$

Magnitude: $\quad 2.9 \mathrm{Mn}(\mathrm{J})$

This earthquake was felt at many towns near Lake Winnipesaukee in the Laconia-Meredith area (press report).

Residents in the Lakeport section of Laconia thought that their furnaces had exploded or they described the sound as similar to a sonic boom (press report). 
Table 2.--Summary of macroseismic data for U. S. earthquakes, Octoher-December 1982--Continued

NEW HAMPSHIRE--Continued

Intensity IV:

New Hampshire--Laconia (Lakeport section) (press report).

Intensity III:

Maine--Eliot.

New Hampshire--Meredith (press report).

Intensity II:

Maine--East Waterboro.

New Hampshire--Hampton, Lebanon.

Vermont--Springfield.

Felt:

Maine--North Fryeburg, Scarborough.

1 December ( $\mathrm{J}$ ) Central New Hampshire

Origin time: 230502.2

Epicenter: $\quad 43.62 \mathrm{~N} ., 71.55 \mathrm{~W}$.

Depth: $\quad 0 \mathrm{~km}$

Magnitude: $\quad 2.2 \mathrm{Mn}(\mathrm{J})$

Felt in the Laconia-Meredith area (press report).

\section{NEW MEXICO}

7 October (G) Central New Mexico

Origin time: 124125.9

Epicenter: $\quad 34.31 \mathrm{~N} ., 106.82 \mathrm{~W}$.

Depth: $\quad 4 \mathrm{~km}$

Magnitude: $\quad 2.4 \mathrm{ML}(\mathrm{G})$

Felt at Socorro.

14 October (G) Texas Panhandle

Origin time: 125246.3

See Texas 1isting.

\section{OREGON}

21 November (G) Northwestern Oregon

origin time: 045732.8

Epicenter: $\quad 45.90 \mathrm{~N} ., 122.89 \mathrm{~W}$.

Depth: $22 \mathrm{~km}$

Magnitude: $2.5 \mathrm{ML}(\mathrm{G})$

Felt in the Woodland, Washington area (press report).
Table 2.-Summary of macroseismic data for U. S. earthquakes. October-December 1982--Continued

RHODE ISLAND

6 November ( J) Northern Rhode Island

Origin time: 035012.4

Epicenter: $\quad 41.79 \mathrm{~N} ., 71.56 \mathrm{~W}$.

Depth: $\quad 2 \mathrm{~km}$

Magnitude: $\quad 1.8 \mathrm{MD}(\mathrm{J})$

A group of three tremors was reported felt and heard in the Crompton area of West Warkwick. The police reported no damage, "just a lot of people shaken up and a lot of houses rattled" (press report).

Intensity IV: Crompton (press report).

15 November (G) Southeastern South Dakota

Origin time: 025822.9

Epicenter: $\quad 43.01 \mathrm{~N} ., 97.85 \mathrm{~W}$.

Depth: $\quad 5 \mathrm{~km}$

Magnitude: $\quad 4.3 \mathrm{Mn}(\mathrm{T}), 4.3 \mathrm{Mn}(\mathrm{G})$

This earthquake was felt over an area of about $19,700 \mathrm{~km}^{2}$ of northeastern Nebraska, southeastern South Dakota, and western Iowa (fig. 11).

The most common report by people in the affected area was a description of the earthquake sound; they thought that their furnaces had exploded.

Intensity V:

Nebraska--

Center--hairline cracks in plaster, stucco, and drywall interior walls; few windows were cracked; few items were thrown from store shelves; few small objects were overturned and fell; few glassware or dishes were broken.

Coleridge--few small objects fell.

Crofton--few items we re thrown from store shelves, few windows were cracked, few small objects were overturned and fell, few glassware or dishes were broken.

Fordyce--hairline cracks in plaster walls, felt by many.

Hoskins--few small objects were overturned and fell.

Niobrara--few items were thrown from store shelves, few small objects were overturned and fell, few glassware or 


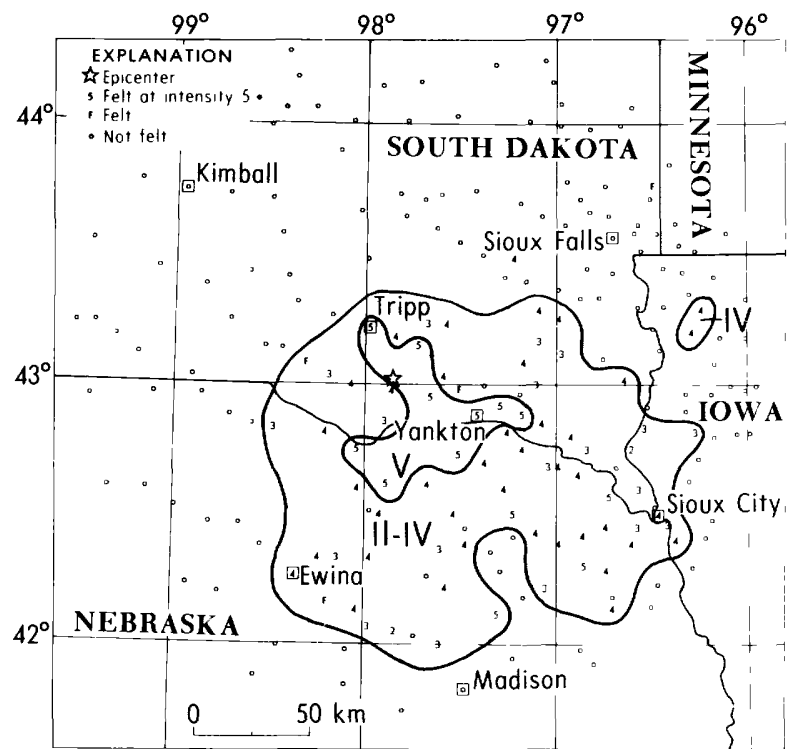

FIGURE 11.--Isoseismal map for the southeastern South Dakota earthquake of 15 November 1982, 025822.9 UTC. Roman numerals represent Modified Mercalli intensities between isoseismals; Arabic numerals are used to represent these intensities at specific sites.

Table 2.--Summary of macroseismic data for U. S. earthquakes. October-December 1982--Continued

\section{SOUTH DAKOTA--Cont inued}

dishes were broken, hanging pictures were swung out of place, felt by all.

Ponca--few small objects fell, felt by many.

Wakefield--few small objects were overturned and fell, few glassware or dishes were broken.

South Dakota--

Gayville--few glassware or dishes were broken, felt by many.

Mission Hil1--few items were thrown from store shelves, few small objects were overturned and fell, hairline cracks in plaster or stucco interior walls, one foundation was reported cracked.

Scotland--few windows were cracked, few small objects were overturned and fe11, felt by all.

Tabor--few small objects fell, hanging pictures were swung out of place, felt by all.

Tripp--few small objects were overturned and fell, hanging pictures were swung out of place.

Yankton--hairline cracks in plaster, stucco or drywall interior walls; few
Table 2.--Summary of macroseismic data for U. S. earthquakes, October-December 1982-.-Continued

SOUTH DAKOTA--Continued

windows were cracked; hanging pictures were swung.

Intensity IV:

Iowa--Doon, Rock Valley, Sergeant Bluff, Sioux City.

Nebraska--Allen, Battle Creek, Bloomfield, Brunswick, Concord, Creighton, Emerson, Ewing, Hartington, Hubbard, Laurel, Maske11, McLean, Neligh, Newcastle (hanging pictures were swung out of place), Orchard, Pender (few small objects fell), Pierce, Saint Helena, Verde1, Verdigre, Waterbury, Wausa, Wynot.

South Dakota--Alcester, Avon, Davis, Hurley, Irene, Kaylor, Lesterville, Lindy (press report), Meckling, Menno, Monroe, Tynda11, Vermillion.

Intensity III:

Iowa--Akron, Brunsville.

Nebraska--Jackson, Lynch, Oakdale, Obert, Osmond, Royal, South Sioux City Wayne.

South Dakota--Burbank, Centerville, Dante, Jefferson, olivet, Springfield, Viborg, Wakonda .

Intensity II:

Iowa-Westfield.

Nebraska--Tilden.

Felt:

Nebraska--Clearwater.

South Dakota--Sherman, Utica (press report), Wagner (press report).

\section{TENNESSEE}

17 October (K) Northwestern Tennessee

Origin time: 195343.0

Epicenter: $\quad 36.24$ N., 89.42 W.

Depth: $\quad 5 \mathrm{~km}$

Magnitude: $2.6 \mathrm{MD}(\mathrm{K})$

Intensity III: Ridgely (K).

\section{TEXAS}

14 October (G) Texas Panhandle

Origin time: 125246.3

Epicenter: $\quad 36.10 \mathrm{~N} ., 102.57 \mathrm{~W}$.

Depth: $\quad 5 \mathrm{~km}$

Magnitude: $\quad 3.9 \mathrm{Mn}(\mathrm{G}), 3.8 \mathrm{Mn}(\mathrm{T})$

The earthquake was described as "just like a sonic boom" and "rumbled like a big train was coming through." 
Table 2.--Summary of macroseismic data for U. S. earthquakes, October-December 1982--Continued

\section{TEXAS--Continued}

Intensity IV:

Texas--Hartley.

Intensity III:

New Mexico--Amistad, Sedan (hanging pictures were swung).

Intensity II:

Texas-Dalhart, Texline.

28 November (G) West Texas

Origin time: 023648.5

Epicenter: $\quad 33.00$ N., $100.84 \mathrm{~W}$.

Depth: $\quad 5 \mathrm{~km}$

Magnitude: $\quad 3.3 \mathrm{Mn}(\mathrm{T})$

Intensity IV: Synder.

Intensity III: 0'Donne11.

Intensity II: Dermott.

\section{UTAH}

14 October (U) Southern Idaho

origin time: 041024.3

See Idaho 1isting.

24 December (G) Southeastern Idaho

Origin time: 151120.1

See Idaho 1isting.

\section{VERMONT}

1 December (J) Central New Hampshire

Origin time: 225223.0

See New Hampshire 1isting.
Table 2.--Summary of macroseismic data for U. S. earthquakes, October-December 1982--Continued

\section{WASHINGTON}

21 November (G) Northwestern Oregon

origin time: 045732.8

See Oregon 1isting.

WYOMING

1 October (G) Yellowstone National Park

Origin time: 225529.6

Epicenter: $\quad 44.33 \mathrm{~N} ., 110.85 \mathrm{~W}$.

Depth: $\quad 5 \mathrm{~km}$

Magnitude: $\quad 3.0 \mathrm{ML}(\mathrm{G})$

Intensity IV: 01d Faithful.

7 October (G) Southeastern Idaho

Origin time: 092602.6

See Idaho 1isting.

8 October (U) Southeastern Idaho Origin time: 095332.1

See Idaho listing.

14 October (U) Southeastern Idaho Origin time: 041024.3

See Idaho 1isting.

8 November (G) Yellows tone National Park Origin time: 011829.3

Epicenter: $\quad 44.78 \mathrm{~N}, 110.92 \mathrm{~W}$.

Depth: $\quad 5 \mathrm{~km}$

Magnitude: $\quad 3.2 \mathrm{ML}(\mathrm{G}), 3.6 \mathrm{MD}(\mathrm{D})$

Intensity III: Madison Junction.

10 November Yellowstone National Park

Origin time: 0340

Epicenter: Not located

Depth: None computed

Magnitude: None computed

Intensity IV: Grant Village. 


\section{ACKNOWLEDGMENTS}

Listed below are the collaborators who furnished data to the U. S. Geological Survey for use in this circular:

\section{ALASKA :}

CALIFORNIA: Clarence R. Allen, Seismological

Staff of National Oceanic and Atmospheric Administration, Alaska Tsunami Warning Center, Palmer. Laboratory, California Institute of Technology, Pasadena.

Bruce A. Bolt, Seismograph Station, University of California, Berkeley.

HAWAII:

Robert Y. Koyanagi, U.S. Geological Survey, Hawaiian Volcano Observatory, Hawaii Volcanoes National Park.

MASSACHUSETTS: Staff of Weston Observatory, Boston College, Weston.

MISSOURI: Robert Herrmann and otto Nutt1i, Department of Geology and Geophysics, St. Louis University, St. Louis.

MONTANA:

Anthony Qamar, University of Montana, Missoula.

NEW YORK: Lynn R. Sykes and Alan L. Kafka, Lamont-Doherty Geological Observatory, Columbia University, Palisades.

OKLAHOMA: James E. Lawson, Jr., Oklahoma Geophysical observatory, oklahoma Geological Survey, Leonard.
TENNESSEE :

UTAH :

VIRGINIA :

WASHINGTON :

A. Johnson, Tennessee Earthquake Information Center, Memphis State University, Memphis. Department of Geology and Geophysics, University of Utah, Salt Lake City.

G. A. Bollinger, Department of Geological Sciences, Virginia Polytechnic Institute and State University, Blacksburg. Robert S. Crosson, Geophysics Program, University of Washington, Seattle.

\section{REFERENCES CITED}

Bath, Markus, 1966, Earthquake energy and magnitude, in Physics and chemistry of the Earth, Volume 7: Oxford and New York, Pergamon Press, p. 115-165.

Gutenberg, B., and Richter, C. F., 1956, Magnitude and energy of earthquakes: Annali di Geofisica, v. 9, no. 1, p. 1-15.

Nutt1i, 0. W., 1973, Seismic wave attenuation and magnitude relations for eastern North America: Journal of Geophysical Research, v. 78 , no. 5, p. 876-885.

Richter, C. F., 1958, Elementary seismology: San Francisco, W. H. Freeman, $768 \mathrm{p}$.

Wood, H. O., and Neumann, F., 1931, Modified Mercalli Intensity Scale of 1931: Seismological Society of America Bulletin, v. 21, no. 4, p. 277-283. 